\title{
Measuring polarizabilities using a Bose-Einstein condensate interferometer
}

\author{
Benjamin Deissler \\ Schifferstadt, Germany
}

M.S., University of Virginia, 2003

Vordiplom, Universität Kaiserslautern, 2001

\author{
A Dissertation presented to the Graduate Faculty \\ of the University of Virginia in Candidacy for the Degree of \\ Doctor of Philosophy \\ Department of Physics
}

University of Virginia

May, 2008

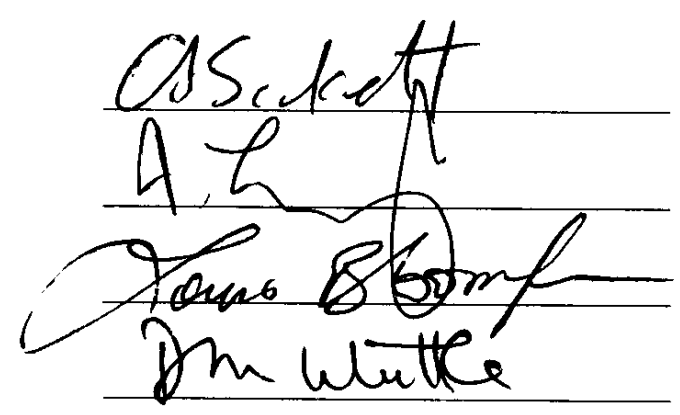




\section{Abstract}

An atom interferometer using Bose-Einstein condensates has been implemented and is used to measure the dynamic polarizability of ${ }^{87} \mathrm{Rb}$. An off-resonant standing-wave laser beam is used to manipulate the motion of atomic wavepackets in order to implement splitting and reflection operations. It is demonstrated that these operations can be performed with near unity fidelity. Interferometer times of up to $72 \mathrm{~ms}$ as well as arm separations of up to $0.42 \mathrm{~mm}$ are achieved experimentally.

The primary limitation for guided-wave atom interferometers with a large arm separation is found to be the effect of the confining potential along the waveguide axis. Such a potential causes a spatially varying phase shift on the wavepackets as they propagate in the interferometer. Different parts of the cloud therefore interfere with different phases, leading to a reduction of the interferometer contrast. A model of the phase shifts on the wavepackets agrees well with the experimental data. In a different scheme in which the wavepackets oscillate freely in the confining potential, these phase shifts completely cancel. Interference is observed at times up to $0.9 \mathrm{~s}$ in this case, but with a fluctuating overall phase of the output, which is attributed to mechanical instabilities.

Finally, the dynamic polarizability of ${ }^{87} \mathrm{Rb}$ is measured. A well-calibrated laser beam is applied to one atomic packet and not the other, inducing a differential phase shift. This technique requires relatively low laser intensity and works for arbitrary optical frequencies. For offresonant light, the ac polarizability is obtained with a statistical accuracy of $3 \%$ and a calibration 
uncertainty of $6 \%$. On resonance, the dispersion-shaped behavior of the Stark shift is observed, but with a broadened linewidth that is attributed to multiple and collective light scattering effects. The resulting nonlinearity may prove useful for the production and control of squeezed quantum states. In general, this work also demonstrates the applicability of condensate interferometry to practical measurements. 


\section{Zusammenfassung}

Ein Atominterferometer mit Bose-Einstein Kondensaten wurde entwickelt und benutzt um die frequenzabhängige Polarisierbarkeit von ${ }^{87} \mathrm{Rb}$ zu messen. Eine stehende Welle, welche von einem nicht-resonanten Laserstrahl erzeugt wird, wird benutzt um die Bewegung von atomaren Wellenpaketen zu manipulieren. Somit werden Operationen realisiert, die analog zu Strahlteiler und Spiegel für Lichtinterferometer funktionieren. Es wird gezeigt, dass diese Operationen mit nahezu perfekter Fidelity ausgeführt werden können. Experimentell werden Wechselwirkungszeiten von bis zu $72 \mathrm{~ms}$ und eine Trennung der Arme des Interferometers von bis zu $0.42 \mathrm{~mm}$ gemessen.

Es wird gezeigt, dass sich für Atominterferometer in einem Wellenleiter mit großer Trennung der Arme die primäre Beschränkung aus der Potentialvariation entlang der Wellenleiterachse ergibt. Ein solches Potential ist die Ursache für eine räumlich variierende Phasenverschiebung der Wellenpakete nach ihrer Propagationszeit im Interferometer. Unterschiedliche Teile der atomaren Wolke überlagern sich daher mit unterschiedlichen Phasen, was zu einer Reduktion des Kontrasts des Interferometers führt. Ein Modell der Phasenverschiebung der Wellenpakete stimmt gut mit den experimentell gemessenen Daten überein. In einem weiteren Versuch, in dem die Wellenpakete im Potentialtopf ohne Reflexionsoperation propagieren, hebt sich diese Phasenverschiebung gerade auf. Interferenz wird in diesem Fall für Interferometer von 0.9 s Länge beobachtet. Allerdings fluktuiert hier die Phase der gesamten Wolke, was unzureichender mechanischer Stabilität der Apparatur zuzuschreiben ist. 
Schließlich wurde die frequenzabhängige Polarisierbarkeit von ${ }^{87} \mathrm{Rb}$ gemessen. Ein Laser mit kalibierter Intensität wird auf ein atomares Wellenpaket geschienen und nicht auf das andere. Dies hat eine relative Phasenverschiebung zur Folge. Diese Methode benötigt eine relativ niedrige Laserintensität und liefert für willkürliche optische Frequenzen ein Ergebnis. Eine Messung mit nicht-resonantem Licht ergab die Polarisierbarkeit mit einem statistischen Fehler von 3\% und einer Kalibrierungsunsicherheit von 6\%. In der Nähe der atomaren Resonanz wurde der Stark Effekt und dessen dispersions-ähnliche Form gemessen, jedoch mit einer verbreiterten Linienbreite. Diese wird Vielfachstreueffekten sowie kollektiven Streueffekten zugeschrieben. Die resultierende Nichtlinearität könnte sich als nützlich für die Erzeugung und Kontrolle von „gequetschten” Zuständen erweisen. Im allgemeinen zeigt diese Arbeit die Möglichkeit, BoseEinstein Kondensate in Atominterferometern für brauchbare Messungen zu benutzen. 


\section{Acknowledgments}

The work described in this dissertation is of such a magnitude that it cannot be carried out by one person alone. It is rather the product of the hard work of a whole group of people, to whom I owe a great deal.

First and foremost I recognize my advisor, Cass Sackett, who not only passed on some of his seemingly unlimited knowledge, but was also an indispensable source of advice in personal issues. I could not have asked for a better mentor and can only strive to live up to his standards.

My colleagues in the lab - Jessica, Ofir, Ken, Jeramy and John - shared with me many frustrating moments when everything seemed to go wrong (see: cracking glass chamber, imploding glass chamber, bursting coils, broken waveguide), but also many successes and laughs. They are largely responsible for keeping the trip to the lab in the morning something to look forward to. I especially need to mention Ofir Garcia, who not only forced me to realize what I did and did not understand while answering his questions, but who was also a partner in hiking the Grand Canyon, watching the World Cup, and in general, simply a great friend.

My teachers throughout the years - Mr. Zschernitz and Mr. Koch in the Gymnasium and Prof. Hotop and Prof. Fleischhauer in Kaiserslautern - deserve my thanks for conveying their love of physics in general and atomic and optical physics in particular.

At the University of Virginia, I have been in the happy situation of being able to exchange equipment and ideas with some very smart people, especially Prof. Tom Gallagher and Prof. Bob Jones, as well as their students. Furthermore, the staff of the physics department has been ex- 
tremely helpful with all manners of problems small and large.

In addition to academic support, many other people have worked to keep me sane. In Virginia, a large group of friends came from the Movie Club, whose company I will surely miss. In Kaiserslautern, I still fondly remember the times spent with the Ex-FiPS group of Martin, Dirk, Thomas, Gustav, Christian, and Christine. I am certain that in the future, at least one of these people will have some theorem or effect named after them. Finally, I am grateful to the friends that have been a part of my life even though an ocean separates us - Marc, Uli, Dennis, Mario, Karin, Désirée, and all the rest.

There is not much I can say to Kristen that I have not said to her already. She is the reason I am writing this today, and I am certain our adventure will continue in Italy.

My parents are a big part of my life, and will continue to be so. They are the reason I am who I am today, and deserve all my love and thanks.

Finally, I acknowledge financial support from the German Academic Exchange Service (DAAD) and the Graduate School of Arts \& Sciences. 


\section{Contents}

1 Introduction 1

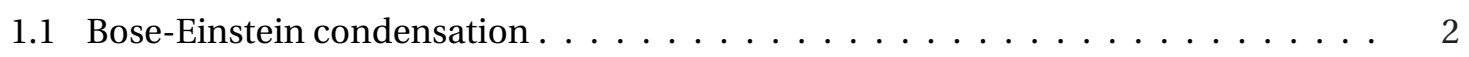

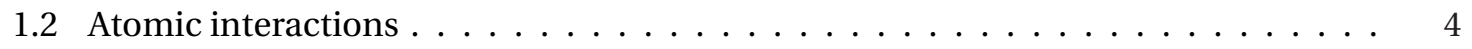

1.3 Atom interferometers $\ldots \ldots \ldots \ldots \ldots \ldots \ldots \ldots \ldots \ldots \ldots \ldots \ldots \ldots \ldots \ldots$

1.4 Why use BEC for interferometry? . . . . . . . . . . . . . . . . . . 10

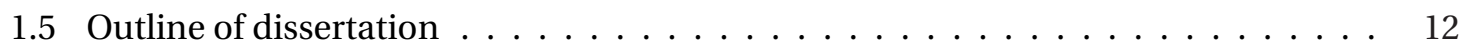

2 Experimental apparatus 114

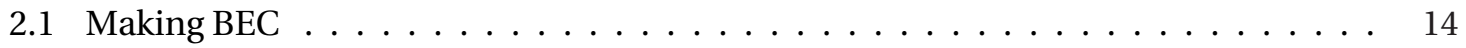

2.1 .1 Magneto-optical trap . . . . . . . . . . . . . . 14

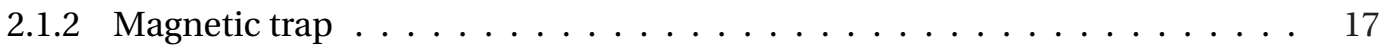

2.1.2.1 Quadrupole trap . . . . . . . . . . . . . 18

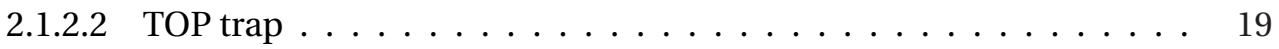

2.1.3 Evaporative cooling .................... 20

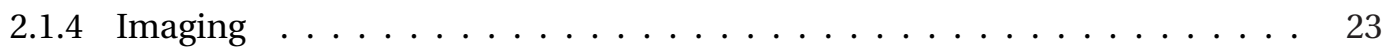

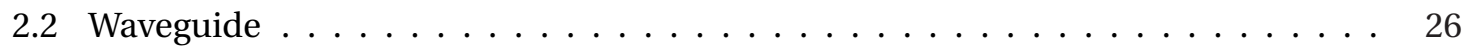

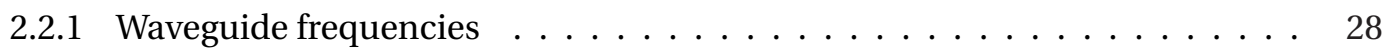

2.2 .2 Experimental setup . . . . . . . . . . . . . . . 29

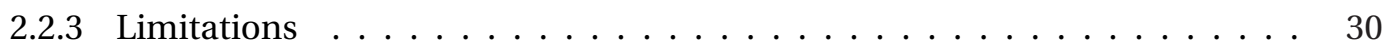


2.2.4 Loading the waveguide $\ldots \ldots \ldots \ldots \ldots \ldots \ldots \ldots$

3 Interferometer 36

3.1 Manipulating atomic motion with standing wave lasers $\ldots \ldots \ldots$. . . . . . 37

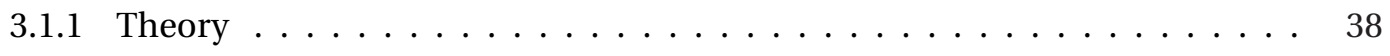

3.1.1.1 Two-level system . . . . . . . . . . . . . . . . . 38

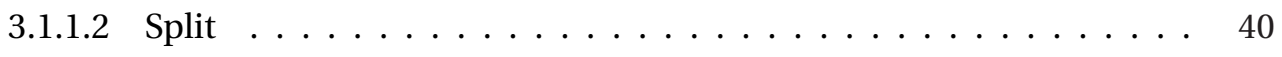

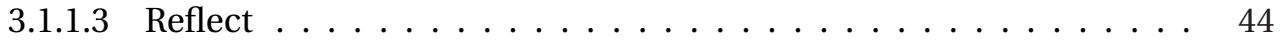

3.1.1.4 Recombine ................ 45

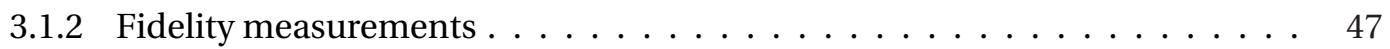

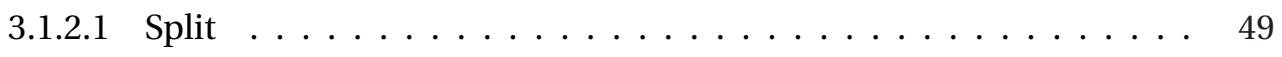

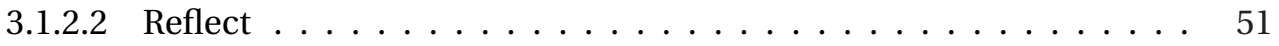

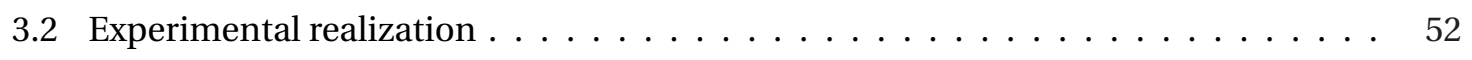

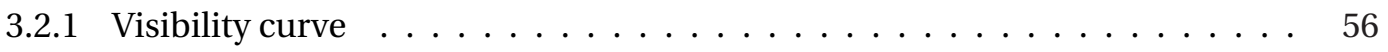

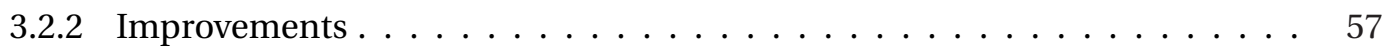

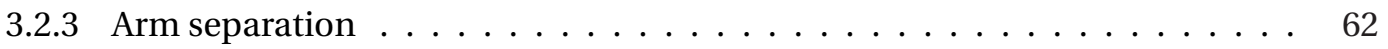

3.3 Limitations . . . . . . . . . . . . . . . . . . 64

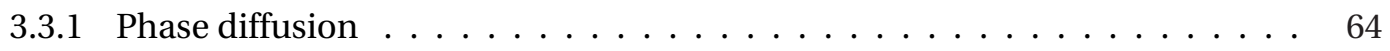

3.3 .2 Atomic interactions . . . . . . . . . . . . . . 67

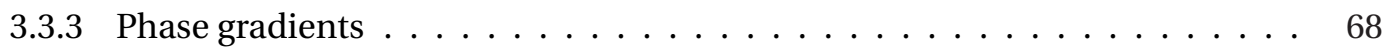

3.3.3.1 Single-sided interferometer $\ldots \ldots \ldots \ldots \ldots$

3.3.3.2 Double-sided interferometer . . . . . . . . . . . . 78

3.3.3.3 Free-oscillation interferometer . . . . . . . . . . 82

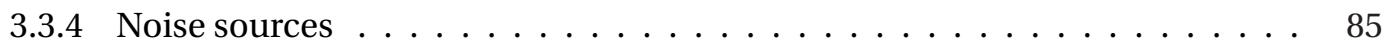

3.3.4.1 Trap field noise $\ldots \ldots \ldots \ldots \ldots \ldots$

3.3.4.2 Motion of trap structure . . . . . . . . . . . . 87

3.3.4.3 External field noise . . . . . . . . . . . . . . 88

3.3.4.4 Residual motion of atoms . . . . . . . . . . . . . . . 89 
3.3.4.5 Bragg beam noise $\ldots \ldots \ldots \ldots \ldots \ldots$

3.3.4.6 Table vibrations . . . . . . . . . . . . . . . . . . . . 91

4 Measuring the ac Stark effect

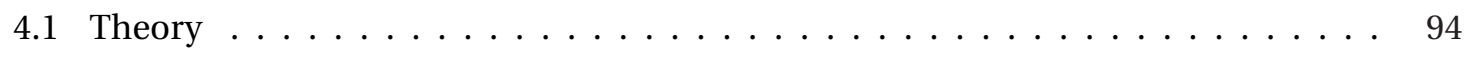

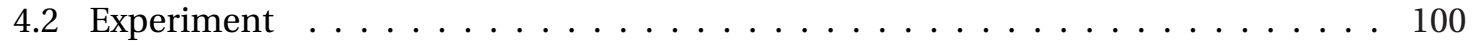

4.2.1 Measurement of the ac polarizability off resonance . . . . . . . . . . 102

4.2.2 Measurement of the ac Stark shift near resonance . . . . . . . . . . . . 106

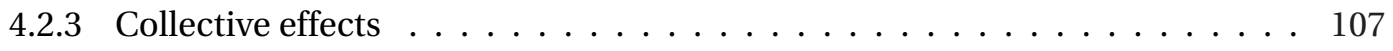

5 Conclusion

5.1 Outlook: Measuring static polarizabilities . . . . . . . . . . . . 116

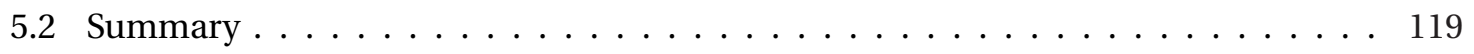

A Rubidium 122

B Canceling the waveguide potential along the guide 124

C Switching circuit

D Capacitor design

Bibliography 138 


\section{List of Figures}

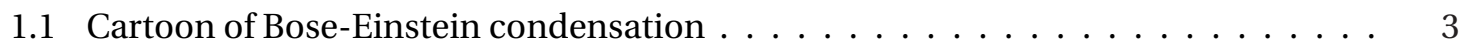

1.2 A depiction of interference $\ldots \ldots \ldots \ldots \ldots \ldots \ldots$

1.3 Schematic of a generic three-grating Mach-Zehnder interferometer . . . . . . . 9

2.1 Schematic of the experimental setup $\ldots \ldots \ldots \ldots \ldots \ldots$

2.2 Schematic of evaporative cooling . . . . . . . . . . . . . . . 21

2.3 Progression of evaporative cooling . . . . . . . . . . . . . . . 22

2.4 Schematic of the imaging system . . . . . . . . . . . . . . . . 23

2.5 Scale drawing of the waveguide structure . . . . . . . . . . . . . . . 29

2.6 Comparison of the measured with the predicted waveguide frequencies $\ldots \ldots$

2.7 Atom position during the waveguide loading sequence . . . . . . . . . . 33

3.1 Setup of the standing-wave laser beam $\ldots \ldots \ldots \ldots \ldots \ldots \ldots$

3.2 Illustration of the Bloch vector precession during the optimized double pulse se-

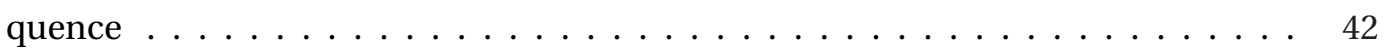

3.3 Fidelity of the splitting operation $\ldots \ldots \ldots \ldots \ldots \ldots \ldots \ldots$

3.4 Fidelity of the reflection operation $\ldots \ldots \ldots \ldots \ldots \ldots \ldots$

3.5 Experimental results for a longer reflection pulse $\ldots \ldots \ldots \ldots \ldots$

3.6 Trajectory of wavepackets in the interferometer . . . . . . . . . 55 
3.7 Calibration of the frequency shift of the standing-wave laser beam . . . . . . . 555

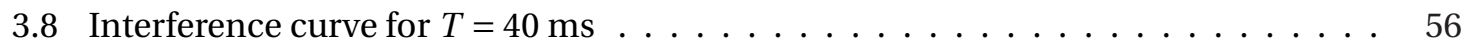

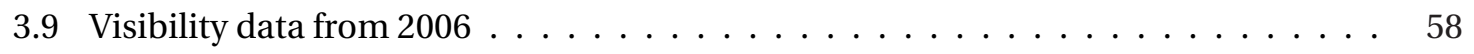

3.10 Images of the beam used to generate the standing wave $\ldots \ldots \ldots \ldots$

3.11 Position of the atoms in the waveguide for several runs of the experiment $\ldots \ldots$

3.12 Visibility after implementation of improvements $\ldots \ldots \ldots \ldots \ldots$

3.13 Separated packets in the interferometer . . . . . . . . . . . . . 63

3.14 Wavepacket trajectories for various interferometer configurations . . . . . . . 69

3.15 Absorption images of wavepackets with a phase gradient in the interferometer . 75

3.16 Experimental results for a single-sided interferometer $\ldots \ldots \ldots \ldots$

3.17 Experimental results for an asymmetric double-sided interferometer . . . . . 79

3.18 Visibility of the symmetric double-sided interferometer $\ldots \ldots \ldots \ldots$

3.19 Interference for atoms freely oscillating in the waveguide $\ldots \ldots \ldots$

3.20 Comparison of the phase noise from various sources $\ldots \ldots \ldots \ldots$

3.21 Comparison of the phase noise from residual motion of the atoms . . . . . . . 89

4.1 Polarizability of the $5 S_{1 / 2}$ ground state of ${ }^{87} \mathrm{Rb} \ldots \ldots \ldots \ldots$

4.2 Schematic of the experimental setup for measurement of the ac Stark shift . . . . 101

4.3 Measurement of the phase shift from an off-resonant laser beam . . . . . . . . . . 102

4.4 Measurement of the phase shift from a laser beam near the atomic resonance . . . 105

4.5 Schematic of light radiated from a collection of atoms. . . . . . . . . . . . . 108

4.6 Atom loss linewidths for various trap configurations. . . . . . . . . . . . . . . 110

4.7 Schematic of the one dimensional model for multiple photon scattering $\ldots \ldots \ldots$

5.1 Schematic of the measurement of the static polarizability $\ldots \ldots \ldots \ldots \ldots$

A.1 ${ }^{87} \mathrm{Rb}$ principal transitions with hyperfine structure $\ldots \ldots \ldots \ldots \ldots$

B.1 Schematic of the positions of the four-wire potential cancellation $\ldots \ldots \ldots$ 
B.2 Calculation of anti-trapping potential frequency for various parameters . . . . 126

B.3 Calculations of the anti-trapping potential $\ldots \ldots \ldots \ldots \ldots \ldots \ldots \ldots$

C.1 Schematic of the main switching circuit . . . . . . . . . . . . . . . 130

C.2 Schematic of the control circuit . . . . . . . . . . . . . . . 131

C.3 Schematic of the power supply interlock circuit $\ldots \ldots \ldots \ldots \ldots$

D.1 Schematic of the parallel plate capacitor $\ldots \ldots \ldots \ldots \ldots \ldots$

D.2 Electric field and gradient for the parallel plate capacitor $\ldots \ldots \ldots \ldots$

D.3 Electric field for various gap sizes $\ldots \ldots \ldots \ldots \ldots \ldots$

D.4 Schematic of the parallel plate capacitor $\ldots \ldots \ldots \ldots \ldots \ldots$ 


\section{List of Tables}

2.1 Parameters for transferring atoms from the MOT into the magnetic trap $\ldots \ldots$

3.1 Parameters for higher order splitting operations . . . . . . . . . . . . 51

4.1 Relative intensities of transitions used in calculating the ac polarizability of the $5^{2} S_{1 / 2}$ ground state of ${ }^{87} \mathrm{Rb} \ldots \ldots \ldots \ldots \ldots$

A.1 Constants relevant to the experiment $\ldots \ldots \ldots \ldots \ldots \ldots \ldots$

C.1 Pin connections on Vector card for switching circuit. . . . . . . . . . . . 131 
Wer Wissenschaft und Kunst besitzt,

hat auch Religion;

wer jene beiden nicht besitzt,

der habe Religion.

Johann Wolfgang von Goethe

\section{Introduction}

The question of whether light is a particle or a wave has been a subject of great debate dating back to the Greek philosophers Pythagoras and Aristotle. The debate became all the more intense when quantum mechanics attributed wave properties to matter as well.

In the 1600's, during the development of experimentally based natural sciences, Christiaan Huygens drew up a theory of the wave-like nature of light, whereas Isaac Newton was a proponent of the corpuscular, or particle-like nature. Newton's point of view was prevalent for many years until the 1800's, when Thomas Young's double-slit experiment showed interference, which was seen as evidence for the wave theory. Following that, James Clerk Maxwell derived a theory of light as propagating electromagnetic waves, and thus completed the first unification of physics.

The wave theory of light seemed well established when in 1901 Max Planck postulated the quantized energy of oscillators when calculating the energy spectrum of black-body radiation. The idea that energy could be emitted only in discrete amounts ("quanta") revolutionized physics and led to the development of quantum mechanics. Further evidence for these quanta was given by Albert Einstein's analysis of the photoelectric effect in 1905.

Finally, in 1924, Louis de Broglie applied these ideas to matter, postulating a wave-like theory with a wavelength inversely proportional to the momentum of the particles. This was soon shown to be valid experimentally, and this theory further strengthened the emerging theory of quantum mechanics and the Copenhagen interpretation, in which a system is described by a 
"wave function." Taking advantage of this, the wave properties of matter can be used in devices to make precise measurements, just like the wave properties of light can be used. One particularly interesting case of this is a Bose-Einstein condensate, which acts like a macroscopic matter wave.

\subsection{Bose-Einstein condensation}

In the 1920's, Satyendra Nath Bose worked on the theory of statistics of photons [1], which he sent to Albert Einstein so that it could be translated into German and published. Einstein, impressed by this paper, expanded the result to the statistics of a gas of bosonic atoms [2]. He found that below a critical temperature $T_{C}$, a finite fraction of the bosonic particles are forced to occupy the lowest energy state of the system under consideration. At the transition temperature, the system therefore undergoes a phase transition.

Another, equivalent, way of looking at this is that as atoms of mass $m$ get colder, the thermal de Broglie wavelength at a temperature $T$

$$
\lambda_{\mathrm{DB}}=\left(\frac{2 \pi \hbar^{2}}{m k_{B} T}\right)
$$

becomes comparable to the interparticle spacing $n^{-1 / 3}$, where $n$ is the spatial density of the atoms. The condition for condensation to occur can then be calculated in terms of a critical phase space density [3]

$$
\rho_{C}=n \lambda_{\mathrm{DB}}^{3}=\zeta(3 / 2)=2.612 \ldots
$$

where $\zeta(x)$ is the Riemann zeta function. For alkali atoms, the densities required for BoseEinstein condensation are on the order of $10^{12}-10^{15} \mathrm{~cm}^{-3}$, and the temperatures are less than $1 \mu \mathrm{K}$. We can see that relatively high densities and extremely low temperatures of the atoms are needed to achieve condensation.

In general in experiments, the atoms are held in a trap in order to confine them and support 

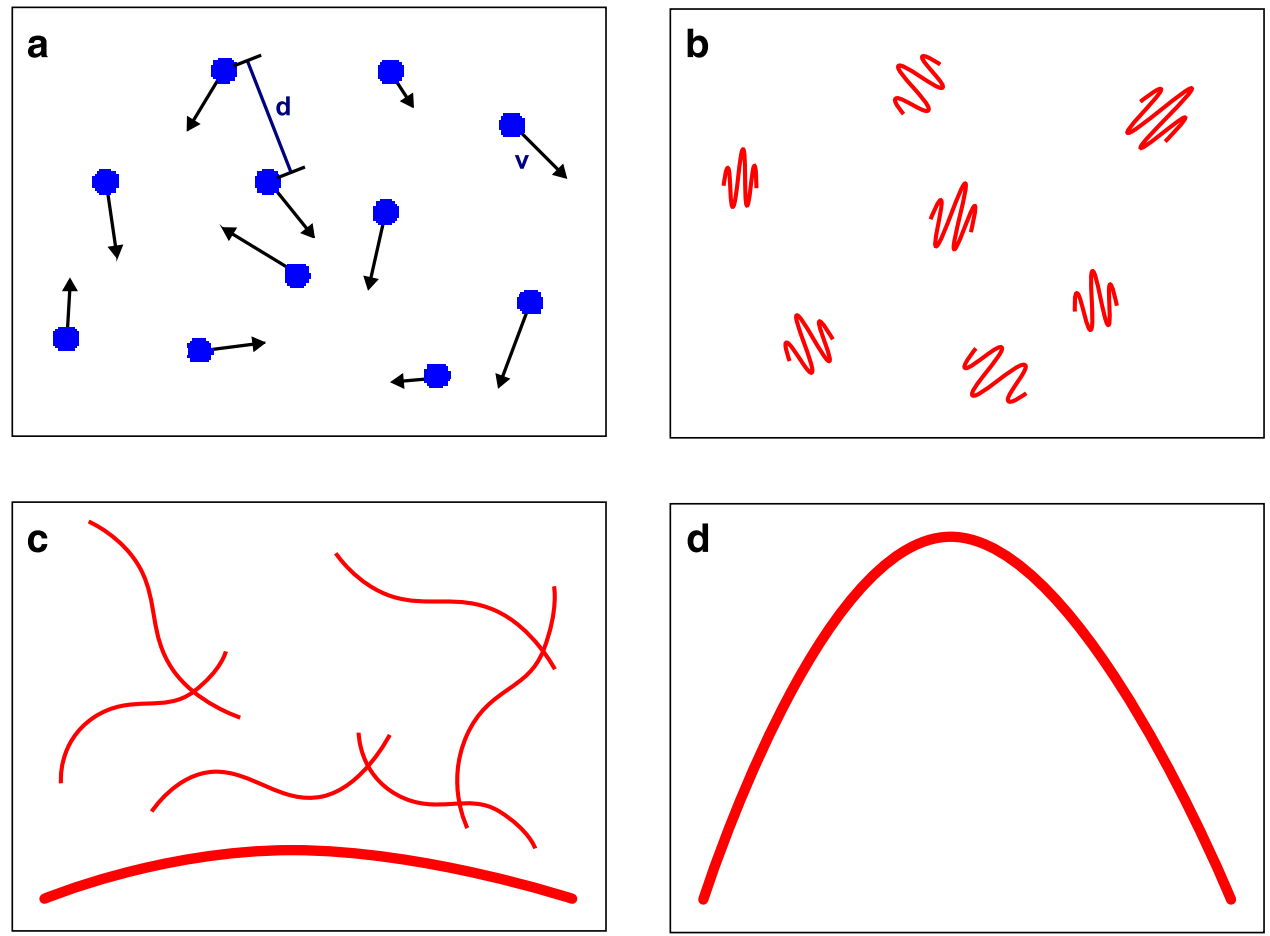

Figure 1.1: Cartoon of Bose-Einstein condensation. (a) At room temperature, particles move with velocity $v$ and have a mean interparticle spacing $d$. (b) As the temperature is decreased, the de Broglie wavelength becomes longer and the wave properties of matter become more pronounced. (c) At the condensation temperature $T_{C}$, the de Broglie wavelength is comparable to the interparticle spacing and a finite fraction of the atoms occupy a single quantum state. (d) If the temperature is decreased even further, almost all the atoms will occupy a single quantum state, having a macroscopic wavefunction.

them against gravity. In most cases, including ours, this is a harmonic trap, and in this case the critical temperature for Bose-Einstein condensation can be expressed [4]

$$
k_{B} T_{C}=\frac{\hbar \omega_{\mathrm{ho}} N^{1 / 3}}{[\zeta(3)]^{1 / 3}}=0.94 \hbar \omega_{\mathrm{ho}} N^{1 / 3},
$$

where $\omega_{\text {ho }}=\left(\omega_{x} \omega_{y} \omega_{z}\right)^{1 / 3}$ is the geometric mean of the confinement frequencies of the harmonic trap.

After the first observation of Bose-Einstein condensation in $\mathrm{Rb}$ [5], $\mathrm{Na}$ [6], and Li [7], there has been a multitude of research conducted with condensates, and quantum degenerate gases in general. The interest in these systems has arisen due to possibilities for use as quantum sim- 
ulators [8], quantum computing, slow light using electromagnetically induced transparency [9], and models for condensed matter physics such as high- $T_{C}$ superconductors [10], Mott insulators [11], or Bose glasses [12]. Furthermore, the possibility of using condensates in atom interferometers was soon realized, and is an active research topic in a number of groups.

\subsection{Atomic interactions}

Whereas with light, interactions among photons and with the environment are in most cases negligible, an important topic in dealing with ultracold gases is atomic interactions and atomic scattering. An understanding of atomic scattering processes and interactions is needed not only for understanding the interferometer operation, but also for evaporative cooling and the production of BEC.

Two interacting particles can be described by an interaction potential $U(R)$, where $R$ is the separation of the two particles $[13,14]$. We describe the incoming wavefunction of the relative motion of the particles via a superposition of partial waves, each having angular momentum $\ell$. In spherical coordinates, we can then write the time-independent Schrödinger equation with the Hamiltonian

$$
H=-\frac{\hbar^{2}}{2 \mu R^{2}} \frac{d}{d R}\left(R^{2} \frac{d}{d R}\right)+\frac{\hbar^{2} \ell(\ell+1)}{2 \mu R^{2}}+U(R)
$$

where $\mu$ is the reduced mass of the interacting particles. The total scattering cross section is then found to be

$$
\sigma=\frac{4 \pi}{k^{2}} \sum_{\ell=0}^{\infty}(2 \ell+1) \sin ^{2} \delta_{\ell}
$$

where the wavevector $k=\sqrt{2 \mu E} / \hbar$ and $\delta_{\ell}$ is the phase shift between incoming and outgoing wavefunction for large $R$.

In a simple case, the effective interaction potential can be approximated by a central poten- 
tial combined with the centrifugal term from the Hamiltonian (1.4) to be [13]

$$
U_{\text {eff }}(R)=-\frac{C_{n}}{R^{n}}+\frac{\hbar^{2} \ell(\ell+1)}{2 \mu R^{2}}
$$

where for two $S$-state atoms (as in our case), the interaction is a van der Waals interaction with $n=6$. For cold atoms (i.e. low energy), the centrifugal barrier prevents all but the $\ell=0$ partial waves from scattering. For sufficiently low energies, all scattering can then be limited to one partial wave, and is referred to as $s$-wave scattering.

For low energies, we then see from Eq. (1.5) that only the phase shift $\delta_{0}$ is important. For low energies $k \rightarrow 0$, the phase shift $\delta_{0}$ behaves as $-k a$, where $a$ is the scattering length [15]. It is then defined as [13]

$$
a=-\lim _{k \rightarrow 0} \frac{\delta_{0}}{k}
$$

An equivalent interpretation of the scattering length is given by Leggett [16]. For the case $k \rightarrow 0$ and large $R$, the s-wave solution to the time-independent Schrödinger equation has the general form

$$
\Psi \propto \frac{\sin [k(R-a)]}{R} .
$$

At $R \approx 0$, the behavior of the wavefunction is complicated, due to the influence of the interaction potential. The scattering length is thus found when comparing the unperturbed wavefunctions with the scattering wavefunctions at long range. The intersection of the shifted (perturbed) wavefunction with the $R$-axis is just the scattering length. From Eq. (1.8) we see that for $a>0$, the wavefunction is repelled from the origin (repulsive interactions), where as for $a<0$ it is attracted (attractive interactions). By making small changes to the inner part of the scattering potential, the scattering length can therefore be changed by a large amount. Inserting Eq. (1.7) into Eq. (1.5) gives the total cross section as $4 \pi a^{2}$, and accounting for the indistiguishability of bosons [16] adds a factor of 2, giving us the constant cross section

$$
\sigma=8 \pi a^{2}
$$


in the low energy limit. For ${ }^{87} \mathrm{Rb}, a=5.77 \mathrm{~nm}$.

We now turn to the interaction potential in Eq. (1.4). For a cold, dilute gas, we can approximate the interaction potential by a delta function as the effective interaction $[4,16]$

$$
U(\mathbf{R})=\frac{4 \pi \hbar^{2} a}{m} \delta(\mathbf{R})
$$

We can insert this effective potential into the Hamiltonian for the motion of an atom in an external potential $U_{\text {ext }}$. We find the Gross-Pitaevskii equation (GPE), which describes the multiparticle wavefunction of a condensate with mean-field interactions $[4,13,16]$

$$
i \hbar \frac{\partial}{\partial t} \Psi(\mathbf{R}, t)=\left(-\frac{\hbar^{2} \nabla^{2}}{2 m}+U_{\mathrm{ext}}(\mathbf{R})+\frac{4 \pi \hbar^{2} a}{m}|\Psi(\mathbf{R}, t)|^{2}\right) \Psi(\mathbf{R}, t) .
$$

We can see that this equation makes sense because the interaction term $U$ will be proportional to the local density of atoms, which itself is proportional to $|\Psi|^{2}$.

\subsection{Atom interferometers}

The concept of interference has been known for over 200 years, and describes the fact that two or more waves (e.g. sound waves or beams of light) overlap and interact in such a way that the intensity of all the waves together is more or less than the sum of all the intensities of the individual waves [17]. This effect can be experienced in person if two speakers are set up facing each other. Moving around in between, the sound is heard louder in some places, quieter in others. A graphical depiction of why this happens can be seen in Fig. 1.2, As cases (e) and (f) illustrate, a condition for interference to occur is that all the waves oscillate in unison.

One of the most frequently seen varieties of interferometry is when light from a single source, such as a laser beam, is split up into two arms, which are later recombined. After the recombination, the intensity of the final beam will vary depending on the difference in conditions between the two arms. Examples of these different conditions could be differences in the lengths of the 

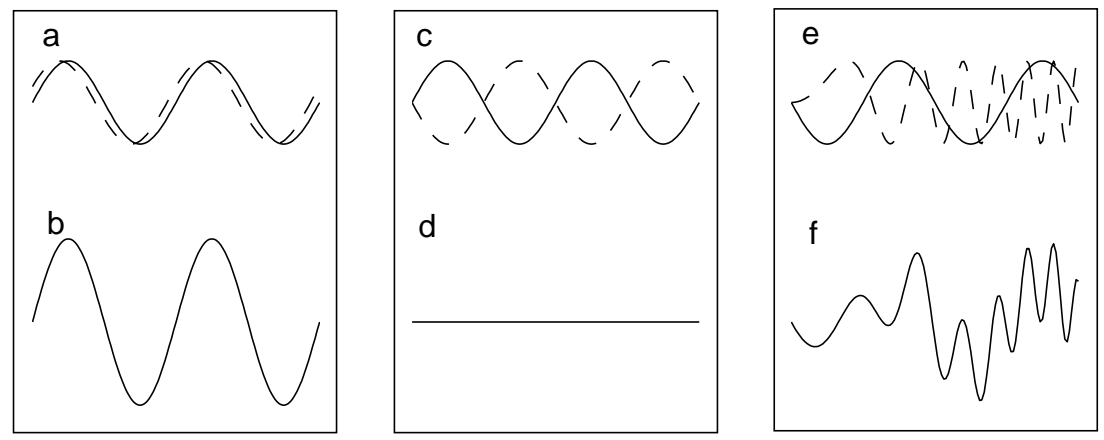

Figure 1.2: A depiction of interference. (a) shows two waves oscillating in unison, (b) shows the interference from these two waves, leading to a single wave with a larger amplitude. (c) and (d) are similar, only this time the two waves cancel each other out. (e) and (f) show what happens when two waves that do not oscillate in unison interfere, in this case the interference effect is less clear than in the previous two cases.

arms or a piece of glass in one arm but not the other.

In order to observe a stable interference pattern, there must be a well-defined phase relation between the interfering waves. An important concept in this respect is coherence. Usually, two types of coherence are differentiated, though they are related. Spatial coherence measures the distance over which the phase of a traveling wave remains constant. Temporal coherence on the other hand denotes the time over which the phase remains constant. Coherence can therefore be used as a measure of how good the interference is - for example, it is easy to see an interference pattern when using a laser, which is a highly coherent source of light, whereas observing interference with a lightbulb is rather difficult.

For the coherence of atomic wavepackets, we can draw an analogy to the interference of optical wavepackets, such as those from a pulsed laser. For a pulsed laser with bandwidth $\Delta v$, the coherence time $t_{C}$ can be approximated by

$$
t_{C} \approx 1 / \Delta v
$$

The coherence length is then simply the distance over which the pulse travels during this time, 
or $l_{C}=c t_{C}$. In an interferometer, this means that if the path length difference is longer than the coherence length, interference cannot be observed. For lasers, this coherence length can be hundreds of meters (or up to the pulse length), whereas for less coherent sources, such as a lightbulb, the coherence length is on the order of a wavelength.

Interferometry has found a multitude of applications. One of the first and most important was an experiment by Michelson and Morley in 1887 to disprove the idea of an "ether" permeating space [18]. This was a critical result leading to Einstein's theory of relativity, with far-reaching consequences. Modern applications are in astronomy, telecommunications, navigation, optical coatings, and holography.

Although the interference of light can be described well with this theory of overlapping waves, other experiments in the early 20th century led to the conclusion that light must have some particle properties as well. This realization was one of the events that triggered the development of quantum mechanics, the best physical theory that exists in science. In 1924, as quantum mechanics was being developed, Louis de Broglie suggested that particles such as atoms have some properties of waves as well [19], with a wavelength $\lambda_{\mathrm{DB}}$ dependent on their momentum $p$ as $\lambda_{\mathrm{DB}}=h / p$, where $h$ is Planck's constant. Shortly thereafter in 1927, Davisson and Germer showed the diffraction of electrons [20], and in 1930 Estermann and Stern measured the diffraction of helium atoms [21]. These experiments were the first to demonstrate the wave properties of atoms, confirming and helping to understand the predictions made by quantum mechanics.

Attempts at using matter waves for interferometry experiments were first successful in the 1950's using electron biprisms [22]. The main limitation of matter-wave interferometers was atom optics, i.e. the manipulation of atomic waves. Whereas light waves are easily manipulated using mirrors and beamsplitters, matter waves require special techniques. In the early 1990's, diffraction from material gratings and diffraction from standing light waves allowed atom interferometers to become feasible $[23,24]$.

The biggest advantage of atom interferometers over light interferometers is that atoms interact with external potentials much more strongly than photons do. Atoms interact readily with 


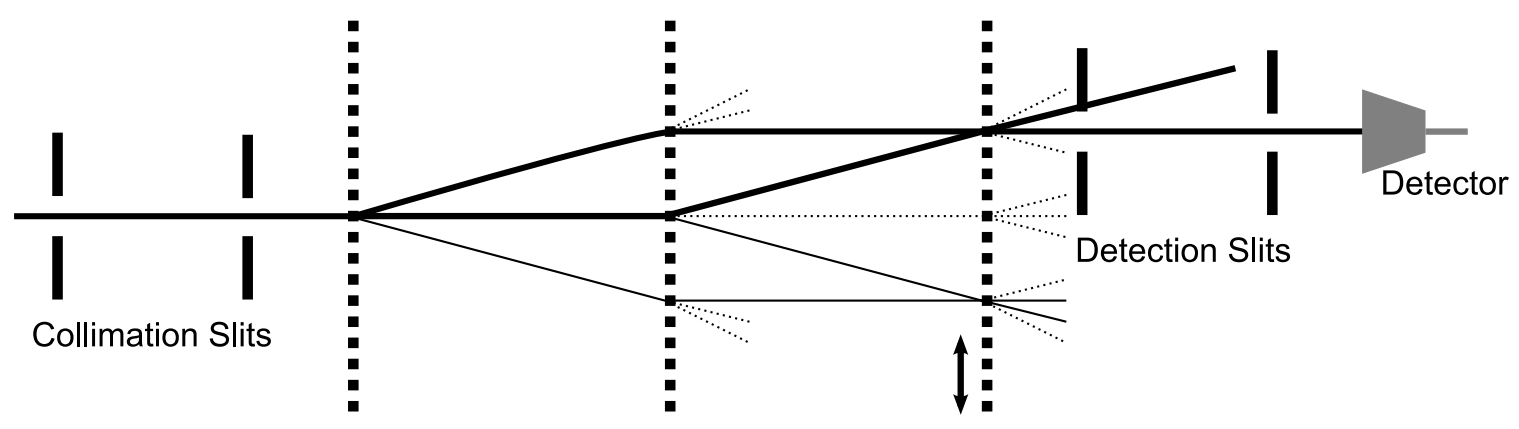

Figure 1.3: Schematic of a generic three-grating Mach-Zehnder interferometer. The waves interfering can be either light waves or matter waves, and the gratings can be either material or from a standing wave of light. The collimation slits define an incoming beam, and the detection slits choose one possible interferometer (thick lines) and output port. The detector measures the output signal. The third grating can be moved relative to the first two, changing the output signal.

electromagnetic fields and gravity, making them ideal for measuring these effects. The phase shift in a light interferometer with a path length difference $\Delta d$ in a medium with index of refraction $n$ is given by $\phi_{\text {light }}=2 \pi n \Delta d / \lambda$, whereas the phase shift in an atom interferometer with an energy difference $\Delta E$ applied for some interaction time $t$ is $\phi_{\text {atoms }}=\Delta E t / \hbar$. One application that has gathered a lot of interest is a measurement of the Sagnac effect $[25,26]$. If the arms of an interferometer enclose an area $A$, then the phase shift due to a rotation with frequency $\Omega$ is given by [27]

$$
\phi=\frac{4 \pi}{\lambda v} \Omega A,
$$

where $\lambda$ is the wavelength and $v$ is the velocity of the waves used in the interferometer. For visible light and middle-weight atoms, the sensitivity of atoms to this effect is about $10^{10}$ higher than for light. A gyroscope with this sensitivity would be able to precisely measure the rotation of the earth, allowing for the development of precise navigation devices. In fact, the best gyroscope on record is an atom interferometer [28].

While the increased sensitivity is promising, this advantage will not be fully realizable. For one, photon sources such as lasers have production rates several orders of magnitude higher 
than atomic beam sources, leading to a better signal-to-noise ratio of the interferometer output. Furthermore, limitations in atom optics mean that only a small range of momenta can be imparted on the atoms, compared to the arbitrary deflections used in light optics. Nonetheless, atom interferometers have proven useful for a variety of applications.

\subsection{Why use BEC for interferometry?}

After the first experimental realizations of BEC in 1995, it was not long before ideas for using condensates in atom interferometers emerged. In a condensate, a macroscopic matter wave is formed, meaning all the atoms are in the same state. Since condensates exhibit a large coherence length on the order of the condensate size of tens of $\mu \mathrm{m}$, condensates can be thought of as analogous to "lasers" if thermal atoms (with coherence lengths on the order of the thermal de Broglie wavelength, around $0.5 \mu \mathrm{m}$ near the condensation temperature) are the analog of "white light." Interference is therefore easily observed, and was first demonstrated by splitting a condensate into two parts and letting them interfere [29]. In the first implementations, the phase of the interference pattern was still random, though, making practical measurements infeasible.

Furthermore, since the atoms in a condensate are so cold and monochromatic, their velocity spread $\Delta v_{\mathrm{BEC}}$ is much smaller than that of thermal atoms. Since the atoms therefore propagate the same path, they have a stable phase relationship, leading to long temporal coherence. A low velocity spread also means that the atoms are more easily manipulated than an atomic beam. The arbitrary deflection angles achievable with cold atoms allow for more flexible geometries, such as large arm separations and enclosed areas. The relatively easy manipulation also means that translational states can be used to separate the arms of an interferometer. In thermal atom interferometers, the separation of arms is often achieved by transferring the atoms into a superposition of internal atomic states [28]. The output is then recovered by measuring the number of atoms in these states, meaning that the atoms are sensitive to the phase of the driving field. This introduces decoherence, limiting the interaction times. Due to the precise control possible 
when using condensates, separating the arms can be achieved using translational states.

However, a big disadvantage of atom interferometers is exacerbated when using condensates. Low production rates were mentioned as reducing the signal-to-noise ratio of atom interferometers versus light interferometers, and for condensates, production rates are even lower. Whereas typical atom beam interferometers have flux rates of $10^{9}$ particles per second, our BEC machine produces about $10^{4}$ atoms in the condensate each minute. Even the best BEC production rates are currently limited to around $10^{5}$ particles per second [30]. A reasonable goal to aim for would be to reach the flux of atomic fountain atom interferometers, which have achieved competitive measurements of the gravitational acceleration [31]. In these experiments, $5 \times 10^{6}$ atoms are involved for a total interaction time of $320 \mathrm{~ms}$, with an experimental cycle lasting for a total time of $1.3 \mathrm{~s}$.

A possible way to compensate for this is to use longer interaction times in the interferometer compared to thermal beam interferometers. We can estimate what interaction times are needed for condensate interferometers to be competitive with thermal atom interferometers by considering the phase shift $\phi=E T / \hbar$ for some energy difference $E$ between the two arms of the interferometer and an interaction time $T$. In the best case, the noise on the output signal $\Delta \phi$ will be limited by shot noise, and therefore $\Delta \phi \approx 1 / \sqrt{N}$. The sensitivity in energy in this case is simply given by $\Delta E \approx \hbar / T \sqrt{N}$. If we use the optimistic estimate of condensate production rates being $10^{-4}$ times those of thermal atoms, then the interaction times of condensate interferometers must be around 100 times longer than those of thermal atom interferometers. These are typically less than $10 \mathrm{~ms}$, so to be competitive, BEC interferometers must aim for interaction times of around one second.

While achieving longer interaction times is difficult, but doable, taking advantage of the easy manipulation of cold atoms leads to another advantage of condensate interferometers. With the ability to separate the arms of the interferometer over a distance larger than the width of the individual clouds, it is possible to individually access the packets. One could then imagine applying an electromagnetic field to one packet but not the other and measuring the resulting 
phase shift. In extracting an atomic property such as the polarizability from a measurement such as this, a condensate interferometer will be able to compete with thermal atom interferometers.

The advantage of spatially separated arms does not eliminate the need to have long interaction times, however, since the coherence times of the interferometer must be large enough to support separating the interferometer arms over large enough a distance. As described in a previous section of this introduction, atomic interactions can cause energy shifts depending on the atom number. Since the splitting operation is expected to have an uncertainty in the atom number on each side on the order of $\sqrt{N}$, a difference in atom number between the two packets will lead to phase noise. Furthermore, when separating the two packets, atoms in one packet can interact with atoms in the other, leading to a spatially varying phase across the packets. Due to these effects, we strive to perform the interferometry experiments in an environment with low atomic density. One way of achieving this is with a very weak confinement, as is realized in the waveguide design presented here.

\subsection{Outline of dissertation}

In this thesis, I will detail the experimental implementation of a guided-wave condensate interferometer and the first practical measurements performed with this device. The work presented herein builds on that presented in the theses of Reeves [32] and Garcia [33], and a portion of the presented matter can be found in more detail in those theses.

An introduction to the experimental setup is found in Chapter 2, First, the production of Bose-Einstein condensates is outlined, and then the waveguide in which the atoms are confined during the interferometer experiments is characterized. In Chapter 3 , the details of the experimental implementation of the interferometer are presented. First, the manipulation of atoms with an off-resonant standing-wave is described theoretically, before measurements of the achieved fidelities of the splitting and reflection operations are shown. Next, the achieved interference curves are described, along with the improvements made to the apparatus after the 
results of [33]. Finally, the limitations of the interferometer - largely due to confinement effects and possible noise sources are given. Chapter 4 presents the main new results of this thesis. The first practical measurement with a guided-wave interferometer was performed by measuring the phase shift due to the ac Stark shift from a beam of light incident on one arm of the interferometer but not the other. In the course of this measurement, an unusual line broadening effect was found, which was then investigated further. Finally, in Chapter 5 I give an outlook on a future experiment that is planned with our apparatus. A measurement of the static polarizability of ${ }^{87} \mathrm{Rb}$ should be possible with an accuracy exceeding that of a conventional atom interferometer.

The results presented in this thesis have largely been previously published in the following publications.

- J. M. Reeves, O. Garcia, B. Deissler, K. L. Baranowski, K. J. Hughes, and C. A. Sackett: "A time-orbiting potential trap for Bose-Einstein condensate interferometry." Phys. Rev. A 72, 051605(R) (2005)

- O. Garcia, B. Deissler, K. J. Hughes, J. M. Reeves and C. A. Sackett: “Bose-Einstein condensate interferometer with macroscopic arm separation.” Phys. Rev. A 74, 031601(R) (2006)

- K. J. Hughes, B. Deissler, J. H. T. Burke and C. A. Sackett: "High-fidelity manipulation of a Bose-Einstein condensate using an optical standing wave.” Phys. Rev. A 76, 035601 (2007)

- J. H. T. Burke, B. Deissler, K. J. Hughes and C. A. Sackett: “Confinement effects in a guidedwave atom interferometer with millimeter-scale arm separation." Submitted to New J. Phys.

- B. Deissler, K. J. Hughes, J. H. T. Burke and C. A. Sackett: “Measurement of the ac Stark shift with a guided matter-wave interferometer." Accepted by Phys. Rev. A 
Damit das Mögliche entsteht, muss immer wieder

das Unmögliche versucht werden.

Hermann Hesse

\section{Experimental apparatus}

The first steps in any of the experiments described later are to make a Bose-Einstein condensate, and then to load the condensate into a specially designed waveguide. In this chapter, I will describe our experimental apparatus to make condensates only briefly, as it has been previously described in detail in the dissertations of Reeves [32] and Garcia [33]. I will then explain why we conduct our experiments in a waveguide and describe the experimental implementation.

\subsection{Making BEC}

We perform all our experiments in a vacuum system consisting of two chambers linked by a thin tube. A schematic of the experimental setup can be seen in Fig. 2.1. We start by making a magneto-optical trap (MOT) in the MOT chamber, where the vacuum pressure is around $2 \times$ $10^{-9}$ Torr. The atoms are then transferred to a magnetic trap and moved to the science chamber, where the pressure is around $3 \times 10^{-11}$ Torr. There, the atoms are evaporatively cooled down to the condensation temperature.

\subsubsection{Magneto-optical trap}

The first step in making a Bose-Einstein condensate is to capture some atoms. We use ${ }^{87} \mathrm{Rb}$ atoms, which we release into the MOT chamber from a pair of getters through which we run $2-$ 


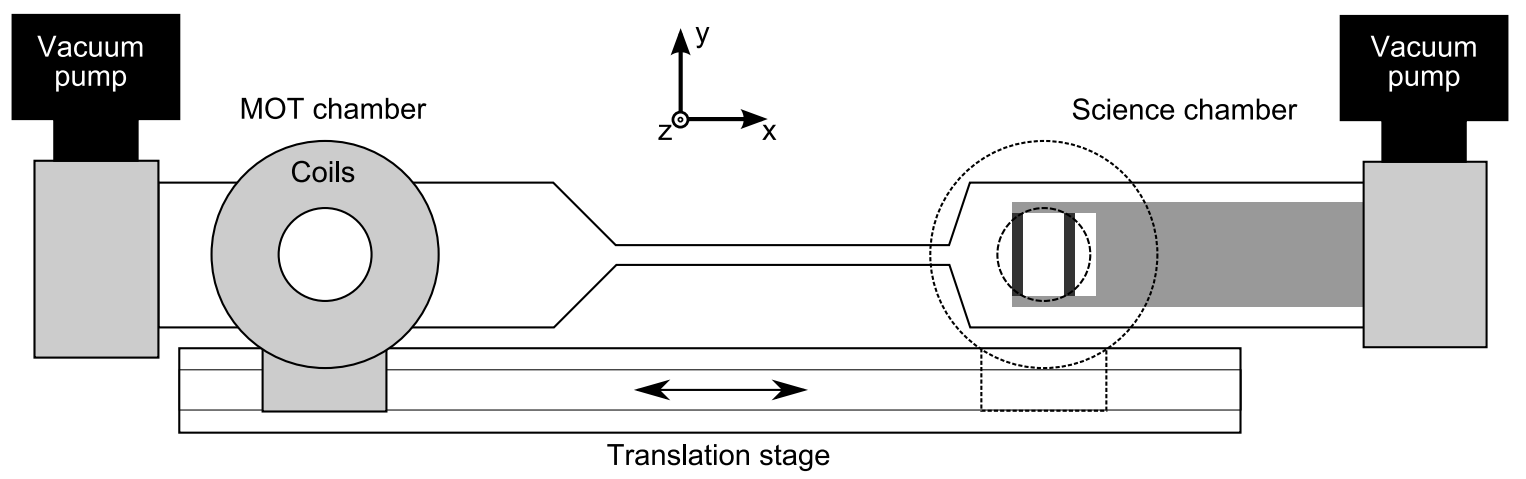

Figure 2.1: Schematic of the experimental setup. Two glass vacuum chambers are linked by a thin tube. In the MOT chamber, atoms are loaded into a MOT before being loaded into a quadrupole magnetic trap. The coils generating the quadrupole field are moved using a translation stage towards the science chamber, which has a better vacuum. The atoms are then transferred into a TOP trap, with a rotating bias field generated by the waveguide structure.

3 A of current. The atoms are then caught in a magneto-optical trap [13]. This consists of 3 pairs of perpendicular laser beams tuned slightly red of the $5^{2} S_{1 / 2}, F=2 \rightarrow 5^{2} P_{3 / 2}, F=3$ transition of ${ }^{87} \mathrm{Rb}$ as well as a smaller amount of light tuned to the $5^{2} S_{1 / 2}, F=1 \rightarrow 5^{2} P_{3 / 2}, F=2$ transition as "repump" light. The magnetic field is a quadrupole field generated by two coils in an antiHelmholtz configuration with a field gradient of $10 \mathrm{G} / \mathrm{cm}$. The MOT normally contains about $3 \times 10^{9}$ atoms at a temperature of around $800 \mu \mathrm{K}$. The phase space density $\rho=n \lambda_{\text {DB }}^{3}$ of the atoms in the MOT is around $10^{-9}$, so we are still several orders of magnitude away from achieving BEC.

In order to get a Bose-Einstein condensate, a near-resonant optical trap such as a MOT cannot be used alone. At the densities that are required, atoms at the edge of a cloud absorb a large fraction of the light, and atoms in the interior of the cloud are therefore not cooled. We must therefore evaporatively cool the atoms, either in a magnetic trap or an off-resonant optical trap [34]. In our experiment, we use a magnetic trap.

In order to load the atoms from a MOT into a magnetic trap, keeping the phase space density as high as possible, the atoms are first compressed in the MOT into a "compressed MOT" (СMOT). This is done by increasing the detuning of the trapping beams and decreasing the power of the repump light. Tuning the laser further off resonance has the effect of decreasing the scat- 
tering rate, i.e. the atoms will not be as optically dense. The increase in magnetic field gradient helps in trapping the atoms, so a higher density of atoms can be achieved. Finally, decreasing the repump power decreases the time the atoms spend in the $F=2$ state resonant with the trapping light [35]. With these steps, the density of atoms in the trap can be increased by reducing the radiation pressure [13]. However, the loading rate of the MOT is also decreased, so we want to apply the CMOT stage for as short a time as possible.

After the atoms have been compressed into a denser cloud in the CMOT, they are optically pumped into the desired state. In our case, we trap the $\left|F=2, m_{F}=2\right\rangle$ atoms in the $5 S_{1 / 2}$ ground state. After the CMOT stage with its decreased repump power, most of the atoms will be in the $F=$ 1 state. Therefore, a short pulse of repump light is applied, as well as a circularly polarized beam of light close to resonance with the $5^{2} S_{1 / 2}, F=2 \longrightarrow 5^{2} P_{3 / 2}, F^{\prime}=2$ transition. In a longitudinal magnetic bias field, the circularly polarized light is $\sigma^{+}$light (when the angular momentum of the light $\ell$ is parallel to the magnetic bias field $\mathbf{B}$ ), which drives $m \longrightarrow m^{\prime}=m+1$ transitions. After being excited, the atoms will reradiate light on one of the $m^{\prime} \longrightarrow m=m^{\prime}, m^{\prime} \pm 1$ transitions. So after a few of these absorption-emission cycles, the atom will find itself in the highest $m$ state (here: $m_{F}=2$ ) and the atom will no longer be able to scatter the light. It is then trapped in the $\left|F=2, m_{F}=2\right\rangle$ ground state.

When turning on the magnetic trap, the size of the atoms in the trap needs to be matched to the size of the cloud after the optical pumping stage. If this is not the case, the size of the cloud undergoes oscillations, leading to heating in the trap. Matching the cloud size can be done by choosing the magnetic field gradient of the magnetic trap in a suitable manner. After initially catching the atoms in the magnetic trap, we can then adiabatically ramp up the magnetic field gradient to the highest value. A table of the transfer procedure is given in Table 2.1, We trap approximately $2 \times 10^{9}$ atoms in the magnetic trap at a temperature of around $900 \mu \mathrm{K}$. 


\begin{tabular}{lcccc}
\hline \hline Stage & Time & Laser detuning & Magnetic gradient & Pumping bias field \\
\hline MOT & $10 \mathrm{~s}$ & $-22 \mathrm{MHz}$ & $10 \mathrm{G} / \mathrm{cm}$ & off \\
CMOT & $30 \mathrm{~ms}$ & $-40 \mathrm{MHz}$ & $10 \mathrm{G} / \mathrm{cm}$ & off \\
Optical pumping & $1 \mathrm{~ms}$ & $-32 \mathrm{MHz}$ & $0 \mathrm{G} / \mathrm{cm}$ & on \\
Magnetic catch & $50 \mathrm{~ms}$ & - & $124 \mathrm{G} / \mathrm{cm}$ & off \\
Magnetic trap ramp & $500 \mathrm{~ms}$ & - & $100 \longrightarrow 387 \mathrm{G} / \mathrm{cm}$ & off \\
\hline \hline
\end{tabular}

Table 2.1: Parameters for transferring atoms from the MOT into the magnetic trap

\subsubsection{Magnetic trap}

Magnetic traps take advantage of the magnetic moments of neutral atoms and the forces exerted on these by inhomogeneous magnetic fields. The energy of an atomic level with angular momentum $\mathbf{F}$ and magnetic quantum number $m_{F}$ in a magnetic field of strength $B$ is [36]

$$
E\left(m_{F}\right)=g \mu_{B} m_{F} B \equiv \mu B
$$

where $g$ is the gyromagnetic moment and $\mu_{B}$ the Bohr magneton. This shows that the potential that an atom sees will be proportional to the strength of the magnetic field. If we use this to trap and cool atoms, the depth of the trap will be on the order of $T=\mu\left(B_{\max }-B_{\min }\right) / k_{B}$, where $T \ll 1 \mathrm{~K}$ for magnetic fields that are conveniently generated. The atoms must therefore be precooled to $\mu \mathrm{K}$ temperatures by the MOT in order to be loaded into a magnetic trap for further cooling. From Eq. (2.1), we see that if $g m_{F}>0$, we have a weak-field seeking state that needs a minimum in $B$ in order to be trapped, while for $g m_{F}<0$, we have a strong-field seeking state, being trapped at a maximum in $B$. It can be shown that no local maxima of $B$ are possible, so only weak-field seeking states can be trapped [37]. We initially load atoms into a quadrupole trap, then after an initial step of evaporative cooling, the atoms are transferred to a time-orbiting potential (TOP) trap. 


\subsubsection{Quadrupole trap}

The quadrupole trap consists of two identical coaxial coils carrying currents in opposite directions (anti-Helmholtz coils) as in the case of the MOT coils. A simple configuration is an axially symmetric trap, which gives a linear potential with

$$
\hat{\mathbf{x}} \cdot \frac{d \mathbf{B}}{d x}=\hat{\mathbf{y}} \cdot \frac{d \mathbf{B}}{d y}=-2 \hat{\mathbf{z}} \cdot \frac{d \mathbf{B}}{d z}
$$

where the condition $\nabla \cdot B=0$ from Maxwell's equations produces the factor of 2 in the $\hat{\mathbf{z}}$ term. The coordinates are defined in Fig. 2.1 The quadrupole trap is generated by a magnetic field

$$
\mathbf{B}(\mathbf{r})=B_{x}^{\prime}(2 z \hat{\mathbf{z}}-x \hat{\mathbf{x}}-y \hat{\mathbf{y}})
$$

which gives a potential

$$
U=\mu|\mathbf{B}|=\mu B_{x}^{\prime} \sqrt{4 z^{2}+x^{2}+y^{2}} .
$$

The quadrupole trap has one major problem. Atoms can undergo a spin flip at a zero point of the magnetic field, a so called Majorana spin flip, and thus fall out of the trap [38]. In a quadrupole trap, these losses are especially important because the zero of the magnetic field is in the center of the trap, where the density of atoms is the highest, and becomes all the more important at lower temperatures, when the density in the center of the trap increases. The lifetime of a cloud with a full width at half max $\sigma_{\text {FWHM }}$ resulting from these losses is found empirically to be

$$
\tau=\frac{1}{4} \alpha \sigma_{\mathrm{FWHM}}^{2}
$$

where $\alpha=3.7(7) \times 10^{4} \mathrm{~s} / \mathrm{cm}^{2}$ for ${ }^{87} \mathrm{Rb}$ [39]. In the experiment, we actually fit the density profile to a Gaussian and measure its $1 / e$-width $w$. Converting the FWHM radius $\sigma_{\mathrm{FWHM}}$ to this width gives

$$
\tau=80 \frac{\mathrm{s}}{\mathrm{mm}^{2}} \cdot w^{2}
$$




\subsubsection{TOP trap}

Though the implementation of a quadrupople trap is extremely simple, losses from Majorana spin flips will limit its usefulness at lower temperatures. Adding a spatially uniform time-independent bias field to this arrangement does not eliminate the zero point, but simply shifts it, so a more elaborate setup is needed. What is desired is, in effect, a potential with a minimum that is non-zero. This can be achieved by adding a time-dependent bias field

$$
\mathbf{B}_{\mathbf{0}}=B_{0}(\hat{\mathbf{x}} \cos \Omega t+\hat{\mathbf{z}} \sin \Omega t)
$$

which is added to the quadrupole field

$$
\mathbf{B}_{\mathbf{q}}=B_{x}^{\prime}(2 z \hat{\mathbf{z}}-x \hat{\mathbf{x}}-y \hat{\mathbf{y}})
$$

to form a time-orbiting potential (TOP) trap [39].

The magnitude of the combined field is then

$$
|\mathbf{B}|=\left[B_{0}^{2}+B_{x}^{\prime 2}\left(x^{2}+y^{2}+4 z^{2}\right)-2 B_{0} B_{x}^{\prime} x \cos \Omega t+4 B_{0} B_{x}^{\prime} z \sin \Omega t\right]^{1 / 2} .
$$

From this equation, we can see that the zero of the magnetic field moves in an ellipse with minor radius $r_{D}=B_{0} / 2 B_{x}^{\prime}$, the radius of the so-called circle of death.

If the oscillation frequency $\Omega$ of the bias field is chosen such that it is smaller than the Larmor frequency $\omega_{L}=\mu B / \hbar \approx 10 \mathrm{MHz}$, the atomic spins can adiabatically follow the field. Choosing $\Omega$ to be larger than the frequency of atomic motion (about $10 \mathrm{~Hz}$ ) means that the atoms will not be able to physically follow the zero of the field. They therefore experience a time-averaged potential. Expanding the square root to second order and taking the time average then gives

$$
\langle|\mathbf{B}|\rangle=B_{0}+\frac{B_{x}^{\prime 2}}{B_{0}}\left(\frac{1}{4} x^{2}+\frac{1}{2} y^{2}+z^{2}\right)
$$


which generates a harmonic potential with a minimum of $\mu B_{0}$. This also gives us the trap depth of the TOP trap. Inserting the radius of the circle of death into Eq. (2.10) we get $U=\mu\left\langle\left|\mathbf{B}\left(r_{D}\right)\right|\right\rangle=$ $\frac{1}{4} \mu\left|B_{0}\right|$, since atoms which reach the circle of death are removed from the trap. In our trap, the maximum trap depth is only about $350 \mu \mathrm{K}$ at a bias field of $20.5 \mathrm{G}$. We can find the oscillation frequencies in the trap $\omega_{i}$ using $U=\mu\langle|\mathbf{B}|\rangle$ and Eq. (2.10) to get

$$
\omega_{x}^{2}=\frac{1}{2} \frac{\mu B_{x}^{\prime 2}}{m B_{0}}, \quad \omega_{y}^{2}=\frac{\mu B_{x}^{\prime 2}}{m B_{0}}, \quad \omega_{z}^{2}=2 \frac{\mu B_{x}^{\prime 2}}{m B_{0}}
$$

The TOP trap is the trap in which we perform the final stages of evaporative cooling and will make the BEC.

\subsubsection{Evaporative cooling}

Cooling the atoms down to the condensation temperature now requires evaporative cooling [40]. This works the same way a cup of coffee cools - the hottest atoms (those with the most energy) are forced to leave. After rethermalizing, the atoms that are left behind are on average cooler than before the cooling. In the atomic clouds used in our experiment, the atomic density is relatively low, leading to low interatomic scattering rates. Since the rethermalization needed happens through this type of scattering, evaporation takes place on longer timescales of around $30 \mathrm{~s}$, and the lifetimes in the trap must be long enough to support this. In the MOT chamber, the lifetime in the quadrupole trap is limited by collisions with hot background atoms to around $4 \mathrm{~s}$. With the quadrupole trap loaded, the quadrupole coils are therefore moved using a translation stage over a distance of about $0.5 \mathrm{~m}$ to the science chamber, where a better vacuum gives lifetimes of around $80 \mathrm{~s}$. During the transfer, most atoms remain in the trap and heating is small. We observe $1.5 \times 10^{9}$ atoms at $1 \mathrm{mK}$ in the magnetic trap on the science chamber side. Also in the science chamber is the waveguide setup, from which we use the rotating bias field to generate the TOP trap.

In our case, the hottest atoms are forced out of the trap using a radio frequency (rf) electro- 

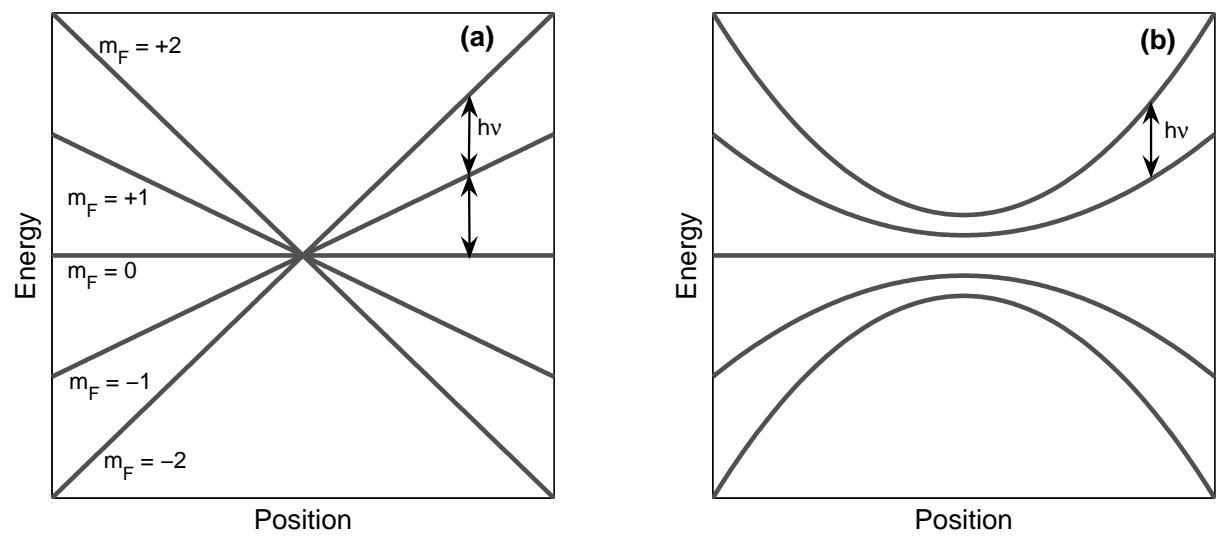

Figure 2.2: Schematic of evaporative cooling. Atoms that have high enough energy to travel to the area in the trap where the rf electromagnetic field is resonant can transition from a trapped to an anti-trapped state and fall out of the trap. (a) Hyperfine levels in the quadrupole trap. (b) Hyperfine levels in the TOP trap.

magnetic field. A schematic of how this works can be seen in Figure 2.2. The atoms are initially in the $m_{F}=2$ hyperfine state. An atom with a high enough energy will be able to move to the side of the trap, where the rf field is resonant with the $m_{F}=2 \rightarrow m_{F}=1$ transition. Falling into this state and subsequently the $m_{F}=0,-1,-2$ states, the atom will be in an untrapped or anti-trapped state and fall out of the trap. The remaining atoms can then rethermalize to a lower temperature.

The frequency of the rf field is swept down during the course of the evaporation, starting with a slow sweep and becoming progressively faster. In order for the evaporation process to be successful, the loss rate of the atoms must be much smaller than the rethermalization rate which is determined by the atomic scattering rate. We determine the evaporative loss rate empirically. In addition to the desired losses that cool the atoms, there are also unwanted losses from collisions with the background vapor, as well as three-body collisions, in which three particles collide and form a diatomic molecule. The binding energy of the molecule is then released into the relative kinetic energy of the molecule and the remaining atom. The total loss rate from the background 

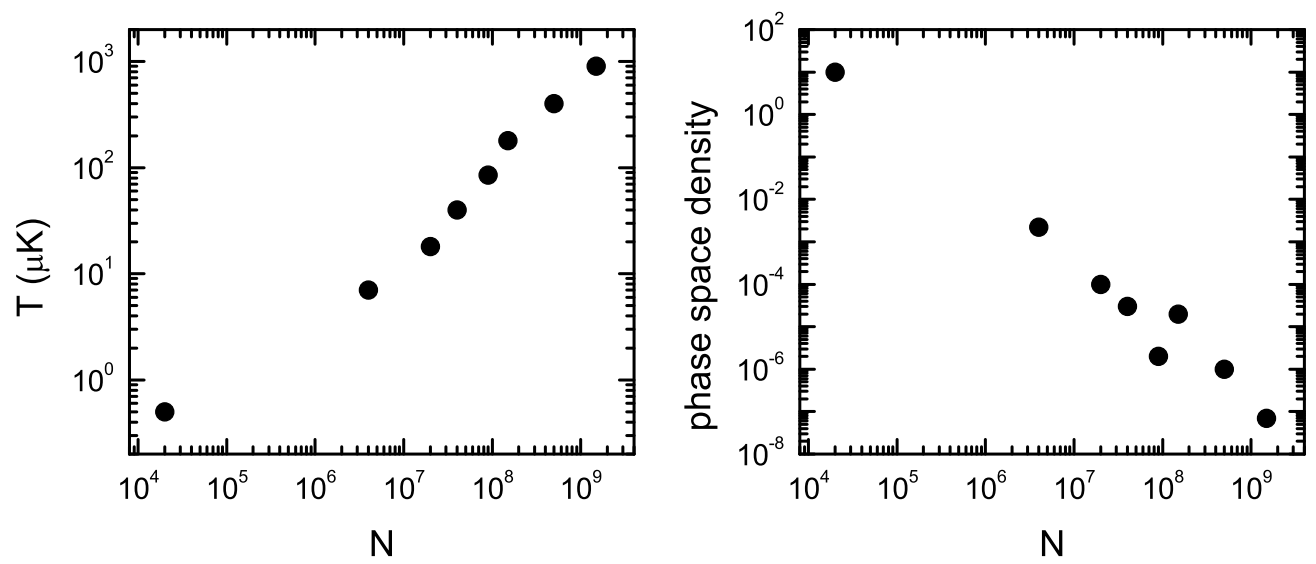

Figure 2.3: Progression of evaporative cooling. The temperature decreases and the phase space density increases as hot atoms are removed from the trap. At $N \approx 10^{8}$, the atoms are transferred from the quadrupole trap to the TOP trap, resulting in a loss of phase space density of around an order of magnitude. This affects the size of the final condensates, which consist of about $3 \times 10^{4}$ atoms.

as well as three-body collision losses is

$$
R_{L}=R_{\text {lifetime }}+R_{3-\text { body }}
$$

where $R_{\text {lifetime }} \approx 1 / 80 s^{-1}$ and $R_{3 \text {-body }}=G_{3} n^{2}$ for atomic density $n$. A measured value of $G_{3}$ is $1.8 \times 10^{-29} \mathrm{~cm}^{6} / \mathrm{s}[41]$.

The frequency sweep of the rf field is programmed as an exponential ramp

$$
v(t)=\left(v_{\mathrm{start}}-v_{0}\right) e^{-t / \tau}+v_{0}
$$

where $\tau$ is the time constant of the sweep and the bottom frequency $v_{0}$ is given by the bottom of our TOP trap as $v_{0}=\mu B_{0} / h$.

Our evaporation consists of three separate steps: We initially evaporate using an rf field in the quadru-pole trap. This gets us to a temperature of around $180 \mu \mathrm{K}$, cold enough to transfer the atoms into the TOP trap. Next, we ramp down the bias field, which decreases the radius of 


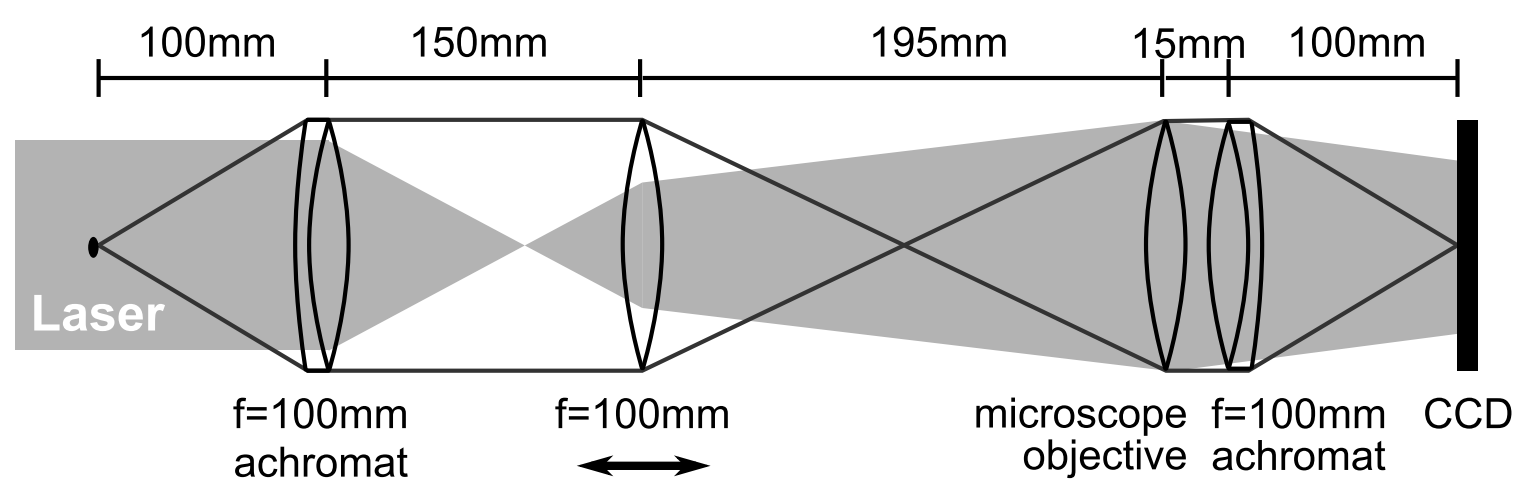

Figure 2.4: Schematic of the imaging system. The black outline shows the ray diagram for the image of the atoms, whereas the gray area shows the path of the laser. Note that the laser beam is focused between the first and second lenses. The second lens is placed on a translation stage to allow focusing of the imaging system. The microscope objective can have a magnification of $2 \mathrm{x}, 5 \mathrm{x}$, or 10x.

the circle of death and also acts as a step of evaporation. Finally, we again use rf evaporation, this time in the TOP trap, in order to achieve Bose-Einstein condensation. We observe condensation with around $3 \times 10^{4}$ atoms at a critical temperature of around $200 \mathrm{nK}$. The relatively low atom number can be explained by the loss in phase space density of around one order of magnitude when we transfer the atoms from the quadrupole trap to the TOP trap, as seen in Fig.2.3.

\subsubsection{Imaging}

Most of the data collected from the experiment is gathered using absorption imaging with a CCD camera. From a picture of a cloud of atoms, we can extract atom numbers, temperatures, and later the interferometer output. Having a well-designed imaging system is therefore paramount for getting good results in our experiments. Since the size of the condensates we are imaging is on the order of a few tens of $\mu \mathrm{m}$, the imaging system must have a large magnification and high resolution. A schematic of the system we use can be seen in Fig.2.4. This system has a measured imaging resolution of $6.6 \mu \mathrm{m}$. The magnification can be varied by changing the microscope objective. 
In absorption imaging, the atoms are illuminated with a short pulse of a weak near-resonant laser beam [42]. The atoms absorb the light and reemit into all directions, causing a shadow of the atomic density profile in the beam. This shadow is what is imaged. It is important to note that this type of imaging is a destructive imaging technique, i.e. the cloud of atoms are lost after imaging. The atoms are released from the trap before imaging to allow the density to decrease in order to avoid saturation of the images, and also to avoid Zeeman shifts of the energy levels. Furthermore, the atoms which absorb and emit a photon undergo an incoherent process and are therefore lost from the condensate. A single image therefore corresponds to one implementation of the experiment.

To get a processed absorption image, we use three raw images. A "background image" is taken without the probe beam on, which captures the ambient light and the CCD offset. Illuminating the atoms with the probe beam gives the "atoms image," whereas probing with the same laser intensity and probe time without the atoms present gives the "no atoms image." The absorption profile can then be obtained by

$$
S(x, y)=\frac{\text { atoms image }- \text { background image }}{\text { no atoms image }- \text { background image }} .
$$

Typical intensities of the probe beam are a few $\mu \mathrm{W} / \mathrm{cm}^{-2}$, and typical probe times are around $50 \mu \mathrm{m}$.

From the measured absorption profile, the number of atoms can be extracted. Calculations of temperature and scattering rate can be found in Refs. [32,33].

Imaging along the $z$-axis, the absorption profile is given by Beer's law as

$$
S(x, y)=e^{-\alpha(x, y)} .
$$

The absorption coefficient $\alpha$ can then be related to the spatial density of the cloud $n$ and the 
light scattering cross section $\sigma_{\mathrm{L}}$ as

$$
\alpha(x, y)=\sigma_{\mathrm{L}} \int n(x, y, z) d z
$$

The general form of an equilibrium density distribution of non-degenerate atoms (i.e. the atoms are above the critical temperature) in a potential $V(\mathbf{r})$ is given by

$$
n(\mathbf{r})=n_{0} e^{-V(\mathbf{r}) / k_{B} T}
$$

where $n_{0}$ is the peak density, $k_{B}$ is Boltzmann's constant and $T$ is the temperature of the sample. For the imaging described in this thesis, we always approximate the density distribution by a Gaussian distribution

$$
n(\mathbf{r})=n_{0} \exp \left(-\frac{x^{2}}{w_{x}^{2}}-\frac{y^{2}}{w_{y}^{2}}-\frac{z^{2}}{w_{z}^{2}}\right) .
$$

Using the normalization of the density distribution to the total number of atoms $N=\int n d \mathbf{r}$, we can find the peak density

$$
n_{0}=\frac{N}{\pi^{3 / 2} w_{x} w_{y} w_{z}}
$$

The integral in Eq. (2.16) can then be calculated as

$$
\int n d z=\frac{N}{\pi w_{x} w_{y}} \exp \left(-\frac{x^{2}}{w_{x}^{2}}-\frac{y^{2}}{w_{y}^{2}}\right) .
$$

Next, we calculate the light scattering cross section $\sigma_{\mathrm{L}}$. Without broadening, the cross section is given as

$$
\sigma_{\mathrm{L}}=\frac{\sigma_{0} \Gamma^{2}}{\Gamma^{2}+4 \Delta^{2}}
$$

where $\Gamma=2 \pi \times 6.065 \mathrm{MHz}$ is the natural linewidth of the $5 S_{1 / 2} \rightarrow 5 P_{3 / 2}$ transition, $\Delta$ is the laser detuning, and $\sigma_{0}=3 \lambda^{2} / 2 \pi$ for $\sigma^{+}$-polarized light or $\sigma_{0}=7 / 15 \times 3 \lambda^{2} / 2 \pi$ for $\pi$-polarized light (the prefactor comes from averaging the coupling strengths for the allowed transitions). In reality, we often find that the linewidth is broadened to $\Gamma^{\prime}$. This broadening does not come from Doppler 
broadening, which would only increase the linewidth by $0.4 \mathrm{MHz}$ for a $1 \mathrm{mK}$ cloud. The broadening is possibly related to the linewidth broadening we have recently observed for cold clouds and condensates at higher optical densities and laser intensities, as described in Sec.4.2.3. From the conservation of the oscillator strength $\int \sigma d \omega$, we can then calculate the reduced cross section $\sigma_{1}$ with $\sigma_{1} \Gamma^{\prime}=\sigma_{0} \Gamma$. The total cross section is therefore

$$
\sigma_{\mathrm{L}}=\frac{\sigma_{1} \Gamma^{\prime 2}}{\Gamma^{\prime 2}+4 \Delta^{2}}=\frac{\sigma_{0} \Gamma \Gamma^{\prime}}{\Gamma^{\prime 2}+4 \Delta^{2}}
$$

Since we are not certain as to what causes this broadening, it it unclear if the assumption that oscillator strengths are conserved is valid. Nevertheless, this provides us with an estimate for the number of atoms. Inserting the calculated values for $\sigma_{\mathrm{L}}$ and $\int n d z$ into Eq. (2.16) and solving for $N$, we find for $\pi$-polarized light

$$
N=\frac{10 \pi^{2}}{7} \frac{\Gamma^{2}+4 \Delta^{2}}{\lambda^{2} \Gamma \Gamma^{\prime}} \alpha_{0} w_{x} w_{y}
$$

We fit the absorption profile to the exponential of a Gaussian function, from which we extract the cloud widths $w_{x}$ and $w_{y}$, as well as the peak absorption $\alpha_{0}$, and from a separate linewidth measurement, $\Gamma^{\prime}$ is known.

\subsection{Waveguide}

Once we have achieved Bose-Einstein condensation, we are almost ready to begin the actual interferometry experiment. As I will describe in this section, we first transfer the atoms from the TOP trap described above to a weakly confining waveguide.

As described in the introduction, one of the limitations of using Bose-Einstein condensates is the low particle flux that can be achieved, and as a consequence for interferometers, long interaction times in the interferometer must be used. In order to reach these long coherence times, atomic interactions must be kept to a minimum. The energy shift from atomic interactions re- 
sults in an additional phase in each packet of the interferometer, which results in a decrease of the observable coherence.

One possibility of avoiding the detrimental effects of atomic interactions is not to use a confining potential. In this case, interference would be measured either by letting two or more packets fall under the influence of gravity, or by using an atomic beam $[43,44]$. In both cases, a large apparatus is needed, either to allow for enough space in the vertical direction, or to allow the atomic beam to propagate. Of course, in the case of a free-space interferometer, stability effects of the guide potential are not a problem, as in the guided-wave case. However, guided atoms allow for long interaction times as well as more flexible geometries, such as larger arm separations and enclosed areas $[45,46]$. One of the advantages of the waveguide described in this section is its stability and minimization of noise, and so we have pursued the guided-wave case.

A way to reduce interactions is to reduce the atomic density, e.g. in a very weakly confining trap. The trap cannot be made arbitrarily weak, however, since the atoms must still be supported against gravity. Simply using a gradient of the magnetic field in one direction to cancel out the potential gradient from gravity is not possible, as it can be shown that a minimum confinement strength is needed in this case [47]. We can, however, combine a very weakly confining harmonic trap with a linear gradient that supports the atoms against gravity.

Furthermore, an interferometer will be sensitive to fluctuating ambient electric and magnetic fields. Most of this noise arises from ac power lines or thermal drifts, so it tends to have low frequencies. In an oscillating field such as the one in a TOP trap, atomic spins adiabatically follow the field. Low-frequency noise will therefore tend to cancel out since the spins see an energy shift with alternating signs. We therefore constructed a waveguide with a weak confining potential and support against gravity with a time-orbiting potential.

TOP traps usually consist of a static quadrupole field combined with a rotating bias field. In this configuration, the zero of the field rotates around the atoms at the center of the trap. In order to get a trap with weak harmonic confinement as well as a support against gravity, we can imagine designing a trap in which the zero oscillates above the atoms at all times, so that they are 
attracted upwards. This can be achieved by not only oscillating the bias field, but the quadrupole field as well.

\subsubsection{Waveguide frequencies}

We already have a rotating bias field in place from the TOP trap, so in the case of the waveguide we add an oscillating quadrupole field. The bias field is given by Eq. (2.7) as

$$
\mathbf{B}_{\mathbf{0}}=B_{0}(\hat{\mathbf{x}} \sin \Omega t+\hat{\mathbf{z}} \cos \Omega t)
$$

to which we add an oscillating quadrupole field

$$
\mathbf{B}_{\mathbf{1}}=B_{1}^{\prime}(x \hat{\mathbf{x}}-z \hat{\mathbf{z}}) \cos \Omega t
$$

The magnitude of these two fields added together can then be written as

$$
\begin{aligned}
|\mathbf{B}| & =\left[\left(B_{1}^{\prime} x \cos \Omega t+B_{0} \sin \Omega t\right)^{2}+\left(-B_{1}^{\prime} z \cos \Omega t+B_{0} \cos \Omega t\right)^{2}\right]^{1 / 2} \\
& =B_{0}\left[1+\left(x^{2}+z^{2}\right) \frac{B_{1}^{\prime 2}}{B_{0}^{2}} \cos ^{2} \Omega t+\frac{2 B_{1}^{\prime}}{B_{0}}\left(x \cos \Omega t \sin \Omega t-z \cos ^{2} \Omega t\right)\right]^{1 / 2} .
\end{aligned}
$$

The square root can be expanded to second order in $B_{1}^{\prime} / B_{0}$, and then the time average is taken to get

$$
\langle|\mathbf{B}|\rangle=B_{0}+\frac{B_{1}^{\prime 2}}{16 B_{0}}\left(3 x^{2}+z^{2}\right)-\frac{1}{2} B_{1}^{\prime} z
$$

To support the atoms against gravity, the gradient of potential energy from the magnetic fields must be equal to the gradient from gravity, i.e. $\mu B_{1}^{\prime} z / 2=m g z$ or

$$
B_{1}^{\prime}=\frac{2 m g}{\mu}
$$

which is around $30 \mathrm{G} / \mathrm{cm}$ for the $|2,2\rangle$ state of ${ }^{87} \mathrm{Rb}$. We get the trap frequencies from this by 


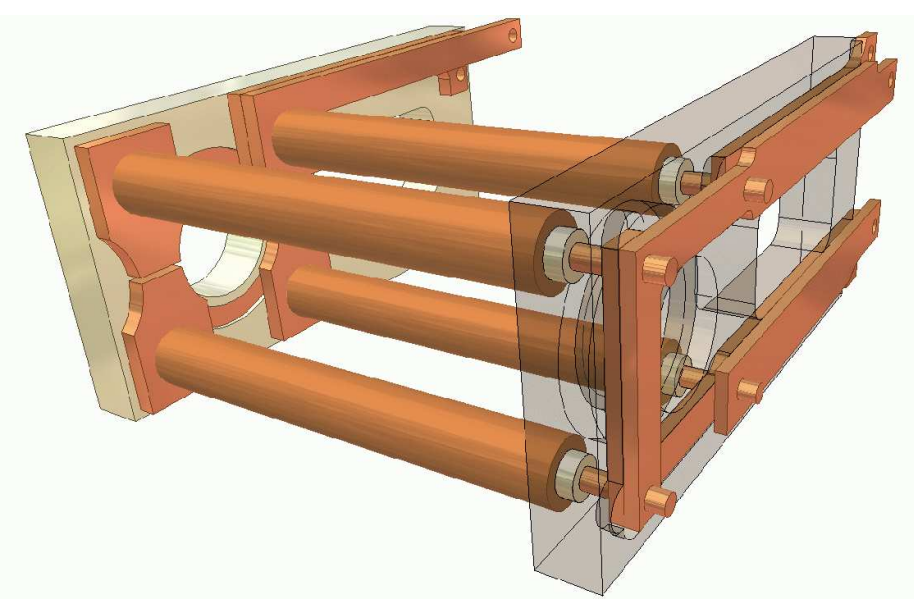

Figure 2.5: Scale drawing of the waveguide structure. The main fields are generated by the four horizontal rods, each of which is a coaxial pair. The rods are held by two boron nitride blocks, which also support the leads and circuit connections. The right block has been depicted as transparent in order to display the arrangement of the conductors. The rod centers form a square $15 \mathrm{~mm}$ on a side, and the blocks are spaced $5 \mathrm{~cm}$ apart.

comparing to a harmonic potential $U=\frac{1}{2} m\left(\omega_{x}^{2} x^{2}+\omega_{z}^{2} z^{2}\right)$ and find

$$
\omega_{x}=\sqrt{\frac{3 m g^{2}}{2 \mu B_{0}}}, \quad \omega_{z}=\frac{\omega_{x}}{\sqrt{3}}=\sqrt{\frac{m g^{2}}{2 \mu B_{0}}} .
$$

\subsubsection{Experimental setup}

The waveguide fields are generated by a 4-rod machined copper structure forming a $1.5 \times 1.5 \times$ $5 \mathrm{~cm}$ block which is situated within the vacuum chamber. The structure is seen in Fig. 2.5. Each of the four rods is actually a coaxial pair. The outer four rods are connected together to generate the waveguide quadrupole field, while the inner conductors form two pairs used to generate the two components of the rotating bias field. The coaxial rods are separated by alumina insulators. The rods are supported by a boron nitride block, which in turn is mounted on $30 \mathrm{~cm}$ long leads which connect to a vacuum flange with feedthroughs to provide the currents. More detail on the waveguide structure can be found in Ref. [32].

The current stability in the waveguide is of utmost importance, since fluctuations of the 
waveguide fields will introduce fluctuations of the phase that degrade the interferometer performance. An actively stabilized circuit using a commercial audio amplifier therefore supplies up to $100 \mathrm{~A}_{\mathrm{pp}}$ for each of the bias fields and the waveguide quadrupole field. The fractional current noise achieved with this is better than one part in $10^{5}$. Details of this circuit are given in Ref. [48]. The rotation frequency of the fields is chosen as $11.9 \mathrm{kHz}$. This frequency must be less than the Larmor precession rate of $\omega_{L}=\mu B / \hbar \approx 10 \mathrm{MHz}$, so that the atomic spins can follow adiabatically, but must be more than the frequency of atomic motion, about $10 \mathrm{~Hz}$. The frequency of $11.9 \mathrm{kHz}$ was chosen to be in between these two values, and is near a minimum of the noise spectrum of ambient noise in our laboratory, since we are most sensitive to noise at the rotation frequency of the fields.

\subsubsection{Limitations}

The results of the previous sections suggest that there is no confinement in the axial ( $y$-) direction. This is not the case, however, due to the finite size of the guide structure and the effects of the leads at the ends. Even though these were designed to minimize axial confinement, they do contribute a small amount of axial confinement. A complete description can be found in Ref. [32]. Briefly, any axial component of the fields from the leads will cause the waveguide quadrupole field to become

$$
\mathbf{B}_{1}=(a x \hat{\mathbf{x}}-c y \hat{\mathbf{y}}-b z \hat{\mathbf{z}}) \cos \Omega t
$$

with $c=b-a$. Including variations in the bias field, the total average field amplitude can be approximated by

$$
\langle|\mathbf{B}|\rangle=B_{0}-\frac{1}{2} b z+\left(\frac{3}{16 B_{0}} a^{2}+\alpha B_{0}\right) x^{2}+\left(\frac{1}{4 B_{0}} c^{2}+\gamma B_{0}\right) y^{2}+\left(\frac{1}{16 B_{0}} b^{2}+\beta B_{0}\right) z^{2},
$$




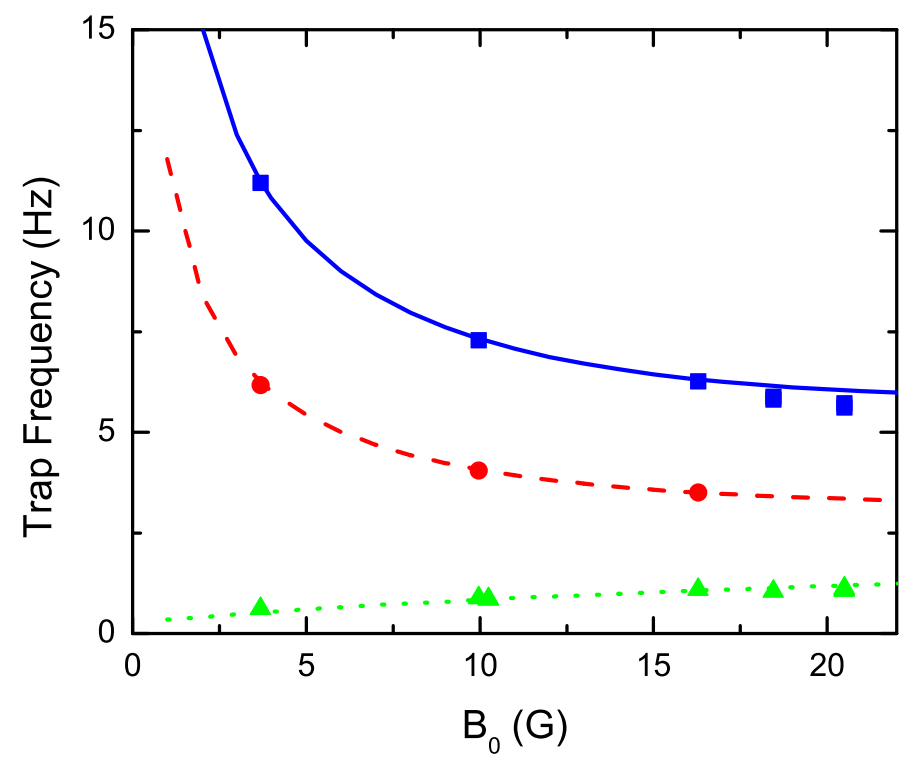

Figure 2.6: Comparison of the measured with the predicted waveguide frequencies. The solid curve gives the frequency in the $x$-direction, the dashed curve in the $z$-direction, and the dotted curve in the $y$-direction. The prediction is from Ref. [32].

which yields frequencies

$$
\begin{aligned}
& \omega_{x}=\left[\frac{2 \mu}{m}\left(\frac{3}{16 B_{0}} a^{2}+\alpha B_{0}\right)\right]^{1 / 2} \\
& \omega_{y}=\left[\frac{2 \mu}{m}\left(\frac{3}{4 B_{0}} c^{2}+\gamma B_{0}\right)\right]^{1 / 2} \\
& \omega_{z}=\left[\frac{2 \mu}{m}\left(\frac{3}{16 B_{0}} b^{2}+\beta B_{0}\right)\right]^{1 / 2} .
\end{aligned}
$$

Here, $a, b$, and $c$ are from Eq. (2.30), and $\alpha, \beta$, and $\gamma$ come from variations in the bias fields. Measuring the trap oscillation frequencies and performing a multivariable fit, we find $|a| / I_{q}=$ $0.734 \mathrm{G} /(\mathrm{A} \mathrm{cm}),|b| / I_{q}=0.709 \mathrm{G} /(\mathrm{A} \mathrm{cm}), \alpha=0.17 \mathrm{~cm}^{-2}, \beta=0.05 \mathrm{~cm}^{-2}$, and $\gamma=0.02 \mathrm{~cm}^{-2}$. A graph of the oscillation frequencies for different bias field strengths can be found in Fig. 22.6, We have measured drifts of the frequency in the axial direction $\omega_{y}$ of up to $5 \%$ over time. This could come from fluctuations in external fields or variations in the guide structure. The lowest confinement is present at a bias field of $20.5 \mathrm{G}$ and has frequencies $\left(\omega_{x}, \omega_{y}, \omega_{z}\right)=2 \pi \times(6,1.2,3.3) \mathrm{Hz}$. 


\subsubsection{Loading the waveguide}

After the production of the condensate in the TOP trap, the atoms are transferred to the waveguide. This happens by performing a series of ramps of the pertinent magnetic fields, in the course of which the external quadrupole field is ramped off, the waveguide quadrupole field is ramped on, and the waveguide bias field is ramped to its final value. We choose the waveguide quadrupole strength such that the atoms are kept in the same position vertically that they had been in the TOP trap. This means that the linear gradient in Eq. 2.27) generated by the waveguide fields is just big enough to support the atoms against gravity.

During the transfer, the condensate spreads out due to the weaker confining potential, therefore becoming less dense, as desired. Due to the weak confinement, extremely low temperatures are possible. For the weakest possible trap and $N=1600$ atoms, the atomic temperature is $850 \mathrm{pK}$, which is to the best of our knowledge the lowest temperature ever observed for Rubidium. We note also that we do not observe any loss of atoms during the transfer into the waveguide.

The most important consideration for the transfer of the condensate into the waveguide is the minimization of oscillations after completion. As we will see later, any residual velocity that the atoms have will lead to a degradation of the optical manipulations used in the interferometer sequence. One of the ways of achieving this is to make the transfer as adiabatic as possible. This implies ramping slowly enough that the atoms can always adiabatically follow the minimum of the instantaneous potential. We find that making the last step of the ramp of the external quadrupole field an exponential ramp to nearly zero greatly reduces subsequent oscillations. The entire sequence of field ramps takes about $7 \mathrm{~s}$ to complete. Furthermore, it is important to align the centers of the waveguide and the TOP trap. Any displacement will make it more difficult to achieve the desired adiabaticity. Since the position of the waveguide is fixed, the TOP trap position is adjusted by moving the external quadrupole field. In the $x$-direction, the final position of the coils along the track is simply adjusted, while in the $y$-direction (along the waveguide axis), the position of the track itself is changed. Most difficult is the $z$-direction, for 


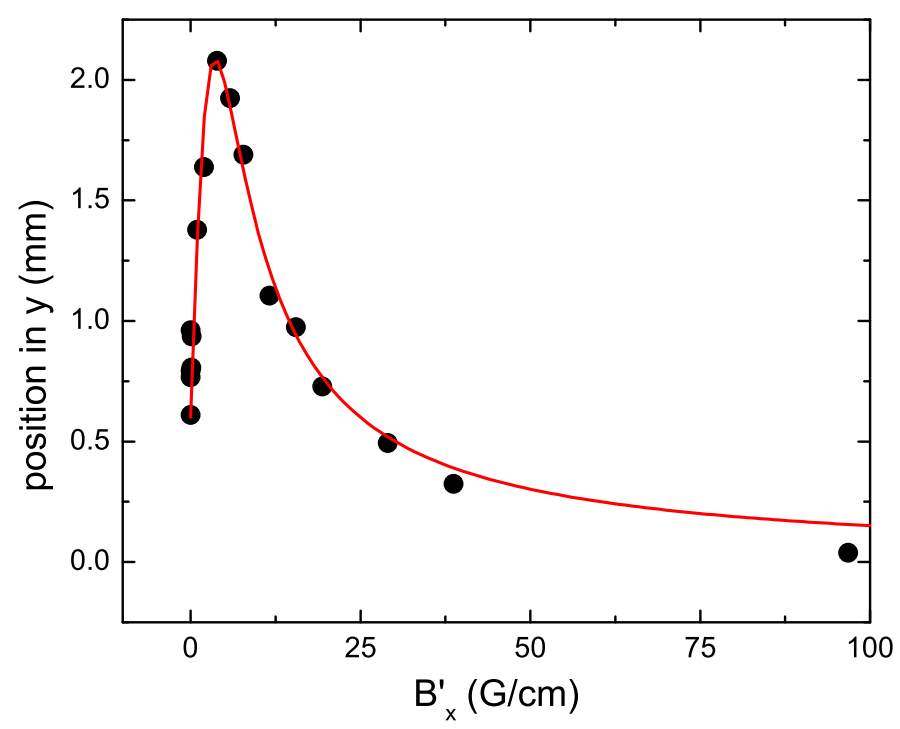

Figure 2.7: Atom position while ramping down the external bias field during the waveguide loading sequence. The dots are the data and the solid curve the theoretical prediction for an offset of $0.6 \mathrm{~mm}$ and a constant bias field of $1.5 \mathrm{G}$.

which the separation of the coils must be changed.

In addition, we found that even with very good alignment of the centers and slow ramps, the atoms still exhibited a small residual velocity. A measurement of cloud position during the loading process showed a sharp deviation from the center shortly before the external quadrupole field is completely ramped off, as seen in Fig. 2.7. We attribute this to a stray external bias field with a component along the $y$-direction. The shift in position can be seen in a model when including a constant bias field

$$
\mathbf{B}_{\mathbf{b}}=B_{b} \hat{\mathbf{y}}
$$

along with the external quadrupole (2.3), the waveguide bias (2.7), and the waveguide quadrupole (2.30). As before, we can expand the magnitude of the combined field to first order and take the time average. The relevant components with a $y$-dependence are then given by

$$
\left\langle\left|\mathbf{B}_{\mathrm{tot}}\right|_{y}\right\rangle=\frac{c^{2}}{4 B_{0}} y^{2}+\frac{B_{b}^{2}}{2 B_{0}}-\frac{B_{b} B_{x}^{\prime}}{B_{0}} y+\frac{B_{x}^{\prime 2}}{2 B_{0}} y^{2} .
$$


However, this includes only the contribution by the waveguide quadrupole field to the confinement along the guide, not the waveguide bias field. Also, the minimum of the waveguide and the external quadrupole trap may not line up, but be displaced by some distance $y_{0}$. To take account of this, we can replace the waveguide confinement term $c^{2} y^{2} / 4 B_{0}$ by $m \omega_{y}^{2}\left(y-y_{0}\right)^{2} / 2 \mu$, where $\omega_{y}$ is the measured trapping frequency along the waveguide. The minimum of the field is then found as

$$
y_{\text {min }}=\left(\frac{B_{b} B_{x}^{\prime}}{B_{0}}+\frac{m \omega_{y}^{2}}{\mu} y_{0}\right) /\left(\frac{B_{x}^{\prime 2}}{B_{0}}+\frac{m \omega_{y}^{2}}{\mu}\right) .
$$

As seen in Fig.2.7 the atoms follow the minimum of the total potential, which is shifted depending on the strength of the external quadrupole field. At first, the strong confinement from the external quadrupole trap dominates, and the zero of the external quadrupole is displaced by $B_{b} / B_{x}^{\prime}$ relative to the center of the quadrupole coils. As the external quadrupole is decreased, the potential minimum moves farther away from its initial position. However, once the confinement from the external quadrupole is on the order of the confinement from the waveguide, the atoms follow the combined minimum of the external field and waveguide. This returns the atoms towards the center of the waveguide, where they end up when no external field is applied. The minimum is therefore only shifted shortly before the external quadrupole field is completely off, causing an increased residual velocity in the final waveguide. This can be compensated for by adding a separate bias coil to cancel this constant bias field and adjusting the current in the bias coil to minimize this deviation of the minimum. We then observe residual velocities of less than $0.2 \mathrm{~mm} / \mathrm{s}$ after the loading procedure.

Another characteristic to note is that even though the physical waveguide structure is level, the wave-guide minimum is not perfectly horizontal, but tilted at a small angle. This needs to be considered later when aligning the standing-wave laser beam used for the interferometer operations to the waveguide axis.

Finally, we observe that the waveguide minimum is at different positions in the $y$-direction at different waveguide bias fields. The change in this direction is on the order of a few $\mathrm{mm}$ between the tightest and weakest confining potentials we use regularly. This could possibly come from 
the effect of gravity on a not perfectly level waveguide, as explained above. Another explanation is that the minimum of the waveguide quadrupole field is not in the same position along the $y$ axis as the minimum of the bias field, causing the combined minimum to vary as the bias field strength is changed. 
Two fixed ideas can no more exist together in the

moral world than two bodies can occupy one and

the same place in the physical world.

Alexander Pushkin

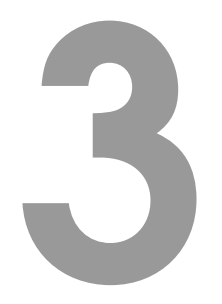

\section{Interferometer}

With the achievement of Bose-Einstein condensation and the implementation of a waveguide, we now have the prerequisite for condensate interferometry experiments. The interferometer scheme used in the following experiments most closely resembles that of a Michelson interferometer. This requires implementing a splitting operation (in analogy to a beamsplitter) and a reflection operation (in analogy to a mirror). In the experiment, these manipulations of the atoms are performed with an off-resonant standing-wave laser beam. In this chapter, these operations will be described, after which the experimental realization is shown and the limitations encountered are explained.

The simplest interferometer scheme that we can think of implementing starts by splitting the Bose-Einstein condensate into two packets traveling in opposite directions with a velocity $v_{0}$. After some amount of time $T / 2$, the two packets are reflected and propagate for another time $T / 2$. At this time, the splitting operation is performed again, but having the opposite effect as before, namely taking the moving atoms and bringing them back to rest. Depending on the relative phase of the two packets in the interferometer before this recombination, some fraction of the atoms will remain at rest and the rest will continue moving away with $v_{0}$. The output of the interferometer can then be measured by imaging the system and extracting the ratio of number of atoms at rest versus the total number of atoms. 


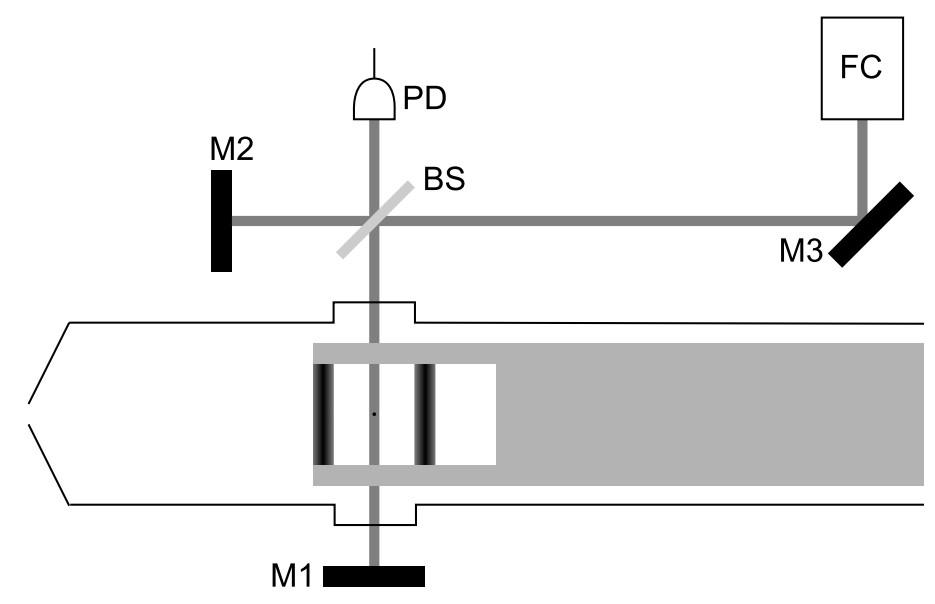

Figure 3.1: Setup of the standing-wave laser beam. The beam comes out of a fiber coupler (FC), is reflected by a beamsplitter (BS) and is retroreflected by the Bragg mirror M1. BS, M1 and mirror M2 act as a Michelson interferometer, the output of which is observed on a photodiode (PD). With this, the stability of the Bragg mirror can be observed.

\subsection{Manipulating atomic motion with standing wave lasers}

The manipulation of matter-waves is a necessary task for atom interferometers, being analogous to the beamsplitters and mirrors in optical interferometers. Many atomic beam interferometers use material gratings, similar to the gratings used in optical interferometers [24]. However, due to the fact that we use a guiding potential and a scheme in which the atoms are at rest initially, this will not work. Instead, we use an optical manipulation scheme of the atoms. An off-resonance standing-wave laser beam generates a periodic potential for the atoms, which can be thought of analogous to a material grating for light waves. This technique has been used to deflect atomic beams [49-51] and has found widespread use in atomic beam interferometers. Cold atom interferometers [52-56] and other experiments requiring the precise manipulation of atomic motion [57-59] use a similar technique, in which the off-resonant standing wave is applied for a certain amount of time to a collection of atoms which is initially at rest.

Here, a theoretical description of the optical manipulation scheme used in the experiment is developed, after which the high-fidelity results of the manipulation of cold atoms is shown. 


\subsubsection{Theory}

Since the atoms in a Bose-Einstein condensate are coherent and all have practically the same velocity, we can approximate the condensate as a plane wave. This allows us to calculate its time evolution with the Schrödinger equation using the translational states of the wavepacket(s). A standing wave of light shifts the energy levels of the atoms by an amount proportional to the intensity of the light [13]. This effect, called the light shift or ac Stark shift, causes the atoms to experience a periodic potential. In the presence of a periodic potential such as that generated by the off-resonant standing wave and neglecting atomic interactions and the guiding potential, the one-dimensional Schrödinger equation can be written as

$$
i \hbar \frac{\partial \Psi}{\partial t}=\left[-\frac{\hbar^{2}}{2 m} \frac{d^{2}}{d y^{2}}+\hbar \beta \cos (2 k y+\alpha)\right]
$$

where $\beta$ is proportional to the amplitude of the standing wave and $\alpha$ is the standing-wave phase.

At low intensities, we consider only the coupling of initial momenta $p_{0}$ to states with momentum $p_{0} \pm 2 \hbar k$. The coupling to higher orders is small enough to be neglected [60]. Assuming the atoms are initially at rest, $p_{0}=0$, we can therefore work in a basis of translational states $\{|0\rangle,|+2\rangle,|-2\rangle\}$, which correspond to packets with momenta $\{0,+2 \hbar k,-2 \hbar k\}$.

In reality, these states correspond to atomic wavepackets, where each wavepacket has a spread of momenta limited by the length of the packet $\Delta p=\hbar / 2 L$. The states are therefore no

longer simply plane waves, but a product $\psi_{0}(y) e^{ \pm 2 i k y}$ of the spatial wavefunction $\psi_{0}$ of each packet and a plane wave $e^{ \pm 2 i k y}$.

\subsubsection{Two-level system}

We will see later that this problem can be simplified to a two-level system. We therefore consider a generic two-level system with a ground state $|g\rangle$ and excited state $|e\rangle$ separated by energy $\hbar \omega_{0}$ interacting with the electromagnetic field of a monochromatic laser beam with Rabi frequency 
$\Omega$ and optical frequency $\omega$. The wavefunction of the two-level system in this case is in general a superposition of ground and excited states

$$
\Psi=c_{g}|g\rangle+c_{e}|e\rangle
$$

with coefficients $c_{g}$ and $c_{e}$ such that $\left|c_{g}\right|^{2}+\left|c_{e}\right|^{2}=1$. The probability of finding the system in the ground (excited) state is then given by $\left|c_{g}\right|^{2}\left(\left|c_{e}\right|^{2}\right)$. The Hamiltonian for this system is given in the $\left\{\left|c_{g}\right\rangle,\left|c_{e}\right\rangle\right\}$ basis by [36]

$$
H=\left(\begin{array}{cc}
0 & \hbar \Omega \cos \omega t \\
\hbar \Omega \cos \omega t & \hbar \omega_{0}
\end{array}\right) .
$$

Applying the rotating wave approximation and defining $d(t) \equiv c_{e}(t) e^{i \omega t}$, we find the effective Hamiltonian in the $\left\{\left|c_{g}\right\rangle,|d\rangle\right\}$ basis

$$
H_{\mathrm{eff}}=\hbar\left(\begin{array}{cc}
0 & \Omega / 2 \\
\Omega / 2 & -\Delta
\end{array}\right)
$$

where $\Delta=\omega-\omega_{0}$ is the detuning of the laser beam from the transition frequency.

The time evolution of the two-level system can then be expressed as $\Psi(t)=U \Psi(0)$, where $U$ is the time-evolution operator $[33,61]$

$$
U=e^{i \Delta t / 2}\left(\begin{array}{cc}
\cos \frac{X t}{2}-i \frac{\Delta}{X} \sin \frac{X t}{2} & -i \frac{\Omega}{X} \sin \frac{X t}{2} \\
-i \frac{\Omega}{X} \sin \frac{X t}{2} & \cos \frac{X t}{2}+i \frac{\Delta}{X} \sin \frac{X t}{2}
\end{array}\right)
$$

Here, $X=\sqrt{\Omega^{2}+\Delta^{2}}$ is the generalized Rabi frequency.

In the case of an additional phase $\alpha$ in the original Hamiltonian, i.e.

$$
H=\left(\begin{array}{cc}
0 & \hbar \Omega \cos (\omega t+\alpha) \\
\hbar \Omega \cos (\omega t+\alpha) & \hbar \omega_{0}
\end{array}\right),
$$


the time evolution operator is calculated in analogous fashion and is found to be

$$
U_{\alpha}=e^{i \Delta t / 2}\left(\begin{array}{cc}
\cos \frac{X t}{2}-i \frac{\Delta}{X} \sin \frac{X t}{2} & -i e^{i \alpha} \frac{\Omega}{X} \sin \frac{X t}{2} \\
-i e^{-i \alpha} \frac{\Omega}{X} \sin \frac{X t}{2} & \cos \frac{X t}{2}+i \frac{\Delta}{X} \sin \frac{X t}{2}
\end{array}\right)
$$

\subsubsection{Split}

We can now consider the effect of the potential generated by an off-resonant standing-wave laser beam with wavenumber $k=\omega / c$ on the atoms.

Our goal is to transfer atoms that are at rest (i.e. in the momentum state $|0\rangle$ ) into two equally populated packets moving apart from each other with equal and opposite momentum $\pm 2 \hbar k$. We label these momentum states $|+2\rangle$ and $|-2\rangle$. The potential of the standing wave can be written as in Eq. (3.1) as

$$
U_{\mathrm{sw}}=\hbar \beta \cos (2 k y+\alpha)
$$

In terms of the momentum states $\{|0\rangle,|+2\rangle,|-2\rangle\}$, the Hamiltonian 3.1 can be written in matrix form by calculating the matrix elements

$$
\begin{aligned}
\langle+2|H| 0\rangle & =\frac{\hbar \beta}{2} e^{i \alpha} \\
\langle-2|H| 0\rangle & =\frac{\hbar \beta}{2} e^{-i \alpha} \\
\langle+2|H|-2\rangle & =0 \\
\langle+2|H|+2\rangle & =4 \hbar \omega_{r},
\end{aligned}
$$

to find

$$
H=\hbar\left(\begin{array}{ccc}
0 & \frac{\beta}{2} e^{-i \alpha} & \frac{\beta}{2} e^{+i \alpha} \\
\frac{\beta}{2} e^{+i \alpha} & 4 \omega_{r} & 0 \\
\frac{\beta}{2} e^{-i \alpha} & 0 & 4 \omega_{r}
\end{array}\right)
$$


Here we have defined the recoil frequency

$$
\omega_{r}=\frac{\hbar k^{2}}{2 m}=2.36 \times 10^{4} \mathrm{~s}^{-1}
$$

where $m$ is the mass of ${ }^{87} \mathrm{Rb}$.

We can now change bases to a basis $|0\rangle_{\alpha},|+\rangle_{\alpha},|-\rangle_{\alpha}$ in which one of the states is not coupled to the other two states. The problem is then again treated as a two-level system. A basis eigenvector $|-\rangle_{\alpha}$ as well as an orthogonal vector $|+\rangle_{\alpha}$ (not an eigenvector) can be found as

$$
|-\rangle_{\alpha}=\frac{1}{\sqrt{2}}\left(\begin{array}{c}
0 \\
e^{i \alpha} \\
-e^{-i \alpha}
\end{array}\right), \quad|+\rangle_{\alpha}=\frac{1}{\sqrt{2}}\left(\begin{array}{c}
0 \\
e^{i \alpha} \\
e^{-i \alpha}
\end{array}\right)
$$

In this basis, the Hamiltonian takes on the desired form in which the $|-\rangle_{\alpha}$ state is decoupled from the other two states.

$$
H=\hbar\left(\begin{array}{ccc}
0 & \frac{\beta}{\sqrt{2}} & 0 \\
\frac{\beta}{\sqrt{2}} & 4 \omega_{r} & 0 \\
0 & 0 & 4 \omega_{r}
\end{array}\right)
$$

We see that the antisymmetric state $|-\rangle_{\alpha}$ is decoupled, and that the $\left\{|0\rangle_{\alpha},|+\rangle_{\alpha}\right\}$ states form a two-level system as described in Sec. 3.1.1.1 with $\Omega=\sqrt{2} \beta$ and $\Delta=-4 \omega_{r}$. For the initial split pulse, the phase of the standing wave does not matter and we can assume $\alpha=0$, in which case the basis vectors are given by the rest state $|0\rangle$, a symmetric state $|+\rangle=\frac{1}{\sqrt{2}}(|+2\rangle+|-2\rangle)$, and an antisymmetric state $|-\rangle=\frac{1}{\sqrt{2}}(|+2\rangle-|-2\rangle)$.

The two-level system is well known, and the time evolution operator is known from Eq. (3.5). A good method of visualization is using the Bloch sphere for the time evolution $[13,62]$. In our case, the Bloch vector is given by $\mathbf{\Omega}=\left(\sqrt{2} \beta, 0,-4 \omega_{r}\right)$, and the ground and excited states are $|0\rangle$ and $|+\rangle$, respectively.

The goal is to transfer all the atoms from the $|0\rangle$ state into the $|+\rangle$ state. We cannot achieve 

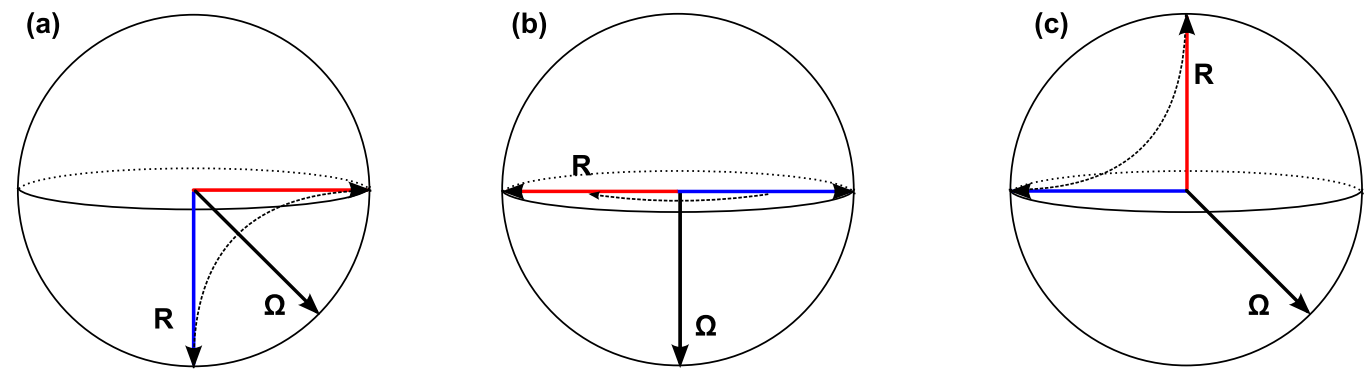

Figure 3.2: Illustration of the Bloch vector precession during the optimized double pulse sequence. (a) During the first pulse, the Bloch vector $\mathbf{R}$ precesses about the Rabi vector $\boldsymbol{\Omega}$ until it reaches the equator of the Bloch sphere. (b) Without the laser beam on, the Bloch vector rotates for half a period along the equator. (c) The second pulse brings the Bloch vector up to the north pole, meaning full population in the $|+\rangle$ state.

this using just a single pulse, since the detuning is fixed to a non-zero $\Delta=-4 \omega_{r}$. However, it is possible to get all atoms into the $|+\rangle$ state by using two pulses, a scheme first worked out by Wu et al. [60]. The first pulse is a $\pi / 2$-pulse, transferring the population into an equal superposition of $|0\rangle$ and $|+\rangle$ states (on the equator of the Bloch sphere). This superposition is then left to propagate freely for half a rotation on the Bloch sphere, after which we apply another $\pi / 2$-pulse to transfer the whole population into the $|+\rangle$ state. We can now calculate the duration and intensity of these pulses as well as the time between pulses.

We use two pulses of equal length $t_{s}$ and intensity $\beta_{s}$, separated by a time $t_{w}$. In a single pulse, we want the state $|0\rangle$ to be transferred to the superposition $\frac{1}{\sqrt{2}}(|0\rangle+|+\rangle)$. By comparing with Eq. (3.5), we see that this is the case for $X t=\pi$ and $\frac{|\Delta|}{X}=\frac{\Omega}{X}=\frac{1}{\sqrt{2}}$, which gives $\Omega=|\Delta|$, $X=\sqrt{2}|\Delta|$, and $t_{s}=\frac{\pi}{\sqrt{2}|\Delta|}$. Inserting these values into the time-evolution operator from Eq. (3.5), we find

$$
U_{s}=-\frac{i}{\sqrt{2}} e^{-i \pi /(2 \sqrt{2})}\left(\begin{array}{cc}
-1 & 1 \\
1 & 1
\end{array}\right) .
$$

For the $180^{\circ}$ precession, we take $\Omega=0$ and find $|\Delta| t_{w}=\pi$, which gives

$$
U_{w}=\left(\begin{array}{cc}
1 & 0 \\
0 & -1
\end{array}\right)
$$


The total time evolution operator is then

$$
U_{\text {split }}=U_{s} U_{w} U_{s}=e^{-i \pi / \sqrt{2}}\left(\begin{array}{ll}
0 & 1 \\
1 & 0
\end{array}\right)
$$

which transfers the atoms from the $|0\rangle$ to the $|+\rangle$ state as desired.

So, in summary, the split pulse parameters are

$$
\begin{aligned}
\beta_{s} & =2 \sqrt{2} \omega_{r} \\
t_{s} & =\frac{\pi}{4 \sqrt{2} \omega_{r}}=23.6 \mu \mathrm{s} \\
t_{w} & =\frac{\pi}{4 \omega_{r}}=33 \mu \mathrm{s},
\end{aligned}
$$

which are modified slightly when contributions from $4 \hbar k$ states are included numerically.

In order to find the experimental intensities, we need to compare the measured intensity of the laser beam to the Rabi frequency we found in the above calculation. First of all, we can measure only the intensity of a single traveling wave, not of a standing wave. The electric field amplitude of a standing wave is twice that of a traveling wave, so the peak intensity of a standing wave is four times that of a traveling wave. We call the measured traveling wave intensity $I_{0}$. For the standing wave we then get

$$
I=4 I_{0} \cos ^{2}(k y)=2 I_{0}(1+\cos (2 k y))
$$

The definition for the saturation intensity relates Rabi frequency and intensity as [13]

$$
\frac{I}{I_{\mathrm{sat}}}=2 \frac{\Omega^{2}}{\Gamma^{2}}
$$

which we can insert into the light shift for large detunings to get

$$
\Delta E_{g}=\frac{\hbar \Omega^{2}}{4 \Delta}=\frac{\hbar \Gamma^{2}}{4 \Delta I_{\mathrm{sat}}} I_{0} \cos (2 k y)
$$


By comparing to Eq. (3.1), we can relate $\beta_{s}$ to the standing-wave intensity and detuning as

$$
\beta_{s}=\frac{\Gamma^{2}}{4 \Delta I_{\mathrm{sat}}} I_{0}
$$

For example, for a detuning of $\Delta=2 \pi \times 12.8 \mathrm{GHz}$, we then find $I_{0}=54 \mathrm{~mW} / \mathrm{cm}^{2}$, where the saturation intensity is $I_{\mathrm{sat}}=3.576 \mathrm{~mW} / \mathrm{cm}^{2}$ for isotropic polarization.

\subsubsection{Reflect}

In addition to a splitting operation, we also need a reflection operation for the interferometer. During the reflection pulse, the atom packets should be turned around, i.e. atoms with momentum $+2 \hbar k$ should have momentum $-2 \hbar k$ after this pulse. In the $\{|0\rangle,|+\rangle,|-\rangle\}$ basis, this corresponds to the state $(|+\rangle+|-\rangle) / \sqrt{2}$ changing into $(|+\rangle-|-\rangle) / \sqrt{2}$. The description as a two-level system is therefore insufficient in this case and we need to consider the $|-\rangle$ state as well. The full time evolution operator in this basis for the interaction of the atoms with a standing wave is

$$
U_{r}=e^{-4 i \omega_{r} t / 2}\left(\begin{array}{ccc}
\cos \frac{X t}{2}+i \frac{4 \omega_{r}}{X} \sin \frac{X t}{2} & -i \frac{\Omega}{X} \sin \frac{X t}{2} & 0 \\
-i \frac{\Omega}{X} \sin \frac{X t}{2} & \cos \frac{X t}{2}-i \frac{4 \omega_{r}}{X} \sin \frac{X t}{2} & 0 \\
0 & 0 & e^{-4 i \omega_{r} t / 2}
\end{array}\right)
$$

So for the reflection, we start with an initial state $\Psi_{i}=1 / \sqrt{2}(0,1,1)$, which evolves into the final state

$$
\Psi_{f}=U_{r} \Psi_{i}=\frac{1}{\sqrt{2}} e^{-2 i \omega_{r} t}\left(\begin{array}{c}
-i \frac{\Omega}{X} \sin \frac{X t}{2} \\
\cos \frac{X t}{2}-i \frac{4 \omega_{r}}{X} \sin \frac{X t}{2} \\
e^{-2 i \omega_{r} t}
\end{array}\right)
$$


The desired state after the reflection is $\Psi_{f}=(0,1,-1)$. This gives the first condition $X t / 2=m \pi$, from which follows $\cos \frac{X t}{2}=(-1)^{m}$, and the final state takes on the form

$$
\Psi_{f}=\frac{1}{\sqrt{2}} e^{-2 i \omega_{r} t}\left(\begin{array}{c}
0 \\
(-1)^{m} \\
e^{-2 i \omega_{r} t}
\end{array}\right)
$$

So in order to get the right form of $\Psi_{f}$, we need $e^{-2 i \omega_{r} t}=-(-1)^{m}=(-1)^{(m \pm 1)}$, as well as $4 \omega_{r}<X$. The simplest solution for these two conditions is $-2 \omega_{r} t=-\pi$ and $X t / 2=2 \pi$. Then the duration of the reflection pulse will be

$$
t_{r}=\frac{\pi}{2 \omega_{r}}=67 \mu \mathrm{s}
$$

and the standing-wave potential amplitude will be

$$
\beta_{r}=2 \sqrt{6} \omega_{r}
$$

which gives an intensity $I_{0}=94 \mathrm{~mW} / \mathrm{cm}^{2}$ for a detuning $\Delta=2 \pi \times 12.8 \mathrm{GHz}$.

\subsubsection{Recombine}

To recombine the atoms, we use the splitting pulse again. The splitting pulse couples the atoms in the $|+\rangle$ state to the $|0\rangle$ state, so if the atoms have not accumulated any phase during their propagation, they will all come back to rest after recombination. In order to measure an interference curve, we need to vary the output of the interferometer in a controlled way. This can be achieved in a similar fashion as in the Mach-Zehnder interferometer depicted in Fig. 1.3 namely by changing the relative phase of the standing wave. We can now look at the recombination pulse as a function of the standing-wave phase $\alpha$ between the splitting and recombination pulses. In 
this case, the standing wave is given by

$$
I=4 I_{0} \sin ^{2} k(y-D)=2 I_{0}(1-\cos 2 k(y-D))
$$

where $D$ is the distance from the mirror to the atoms. This gives us, in analogy to Eq. (3.1), $U_{\mathrm{sw}}=\hbar \beta \cos (2 k y+\alpha)$, so that the phase is given by

$$
\alpha=-2 k D \text {. }
$$

The Hamiltonian of the three-level system in the $\{|0\rangle,|+2\rangle,|-2\rangle\}$ basis is then given generally by Eq. (3.10).

As in Sec. 3.1.1.2, we can change to a basis in which the antisymmetric state $|-\rangle_{\alpha}$ is not coupled to the other two states. The basis vectors in this case are given by Eq. (3.12), and so we can again treat the problem as a two-level system.

During the propagation in the interferometer, the two packets might experience different environments while separated, and will therefore pick up a differential phase $\phi$. Including this differential phase causes the state $|+\rangle$ to become

$$
|\phi\rangle=\frac{1}{\sqrt{2}}\left[e^{i \phi / 2}|+2\rangle+e^{-i \phi / 2}|-2\rangle\right]
$$

We must now express this state in the new basis with the included standing-wave phase $\alpha$ :

$$
\begin{aligned}
|\phi\rangle_{\alpha} & =|+\rangle_{\alpha \alpha}\langle+\mid \phi\rangle+|-\rangle_{\alpha \alpha}\langle-\mid \phi\rangle \\
& =\cos \left(\frac{\phi}{2}-\alpha\right)|+\rangle_{\alpha}+i \sin \left(\frac{\phi}{2}-\alpha\right)|-\rangle_{\alpha}
\end{aligned}
$$

The recombination pulse (same parameters as the splitting pulse) converts this state to

$$
\left|\Psi_{f}\right\rangle=\cos \left(\frac{\phi}{2}-\alpha\right)|0\rangle_{\alpha}+i\left(\frac{\phi}{2}-\alpha\right) \alpha|-\rangle_{\alpha}\left(-e^{-i \pi / \sqrt{2}}\right)
$$


where the factor $-e^{-i \pi / \sqrt{2}}$ comes from the full time propagation operator $U_{\text {split }}=U_{s} U_{w} U_{s}$. The fraction of atoms that comes back to rest is then

$$
\frac{N_{0}}{N}=\left|\left\langle 0 \mid \Psi_{f}\right\rangle\right|^{2}=\cos ^{2}\left(\frac{\phi}{2}-\alpha\right)
$$

\subsubsection{Fidelity measurements}

The splitting and reflecting operations described above can easily be experimentally verified in our apparatus. Bose-Einstein condensates are well-suited for studying interactions of atoms with a standing-wave laser beam due to the fact that all the atoms are essentially moving at the same velocity. Previous studies with atomic beams have been limited by the velocity spread in the beam and the resulting degradation of the operation efficiency [23,24].

We require accurate splitting and reflecting operations in our interferometer. For example, if the splitting operation is inefficient, atoms are left at rest and will subsequently interact with the atoms that are recombined at the end of the interferometer sequence, leading to phase errors and a reduction in visibility. Also, imperfect splitting and reflecting operations lead to a loss of atoms from the wavepackets and therefore a reduced signal-to-noise ratio. In order to verify that our operations work experimentally, we test their dependence on the intensity of the Bragg beam and on the initial velocity of the atoms.

These experiments were performed in a waveguide with frequencies $\left(\omega_{x}, \omega_{y}, \omega_{z}\right)=2 \pi \times$ $(7.4,0.8,4.3) \mathrm{Hz}$. The standing-wave laser was oriented parallel to the $y$-axis (i.e. along the waveguide axis).

As described in Sec.2.2.4 the TOP trap and the waveguide are coincident and the residual velocity of the atoms in the waveguide was reduced to less than $0.2 \mathrm{~mm} / \mathrm{s}$. However, we can also use the loading process to make the atoms oscillate in the wave guide by offsetting the centers of the TOP trap and the waveguide, and abruptly turning off the external quadrupole field before the loading process is complete. This is one of the methods with which the trap frequencies in Sec. 2.2.1 were measured. By performing the splitting and reflection operations at different 
points in the oscillation, we can access different velocities of the atoms. Since both the splitting and reflection operations are much shorter than $1 \mathrm{~ms}$, the atomic velocity is essentially constant during these operations.

The standing-wave laser was tuned to $780.220 \mathrm{~nm}$, about $12.8 \mathrm{GHz}$ blue of the $5 S_{1 / 2} \rightarrow 5 P_{3 / 2}$ transition. At this detuning, the intensity of the split pulse leads to a scattering rate of about $16 \mathrm{~s}^{-1}$, so the number of photons scattered per atom during a pulse of less than $100 \mu \mathrm{s}$ is much less than one and can be neglected. We used an AOM to switch the beam on and off, and to adjust its intensity. An optical fiber was used for spatial filtering and spatial stability, with a maximum output of $12 \mathrm{~mW}$ and a beamwaist of $0.7 \mathrm{~mm}$. After performing the splitting and reflecting operations, the packets are allowed to evolve for around $30 \mathrm{~ms}$, during which they spatially separate, before we image them using absorption imaging.

In general, an image taken after an operation will contain multiple orders $n$ of wavepackets, with velocities $v=v_{i}+n v_{0}$, where $v_{i}$ is the initial velocity and

$$
v_{0}=\frac{\hbar k}{m}=11.7 \frac{\mathrm{mm}}{\mathrm{s}}
$$

is the recoil velocity of the atoms. In this section, we denote the fraction of atoms in each packet as $N_{n}$.

The accuracy of the operation can then be expressed as the fidelity [63]

$$
F=\left|\left\langle\psi_{0} \mid \psi\right\rangle\right|^{2}
$$

where $\left|\psi_{0}\right\rangle$ is the desired state and $|\psi\rangle$ is the observed state. For example, for the split operation, we want $\left|\psi_{0}\right\rangle=|+\rangle$, so for

$$
|\psi\rangle=\sum_{n} c_{n}\left|v_{i}+n v_{0}\right\rangle
$$

the fidelity is

$$
F_{\text {split }}=\frac{1}{2}\left|c_{+1}+c_{-1}\right|^{2}=\frac{1}{2}\left(N_{+1}+N_{-1}+2 \operatorname{Re} c_{+1}^{*} c_{-1}\right) .
$$



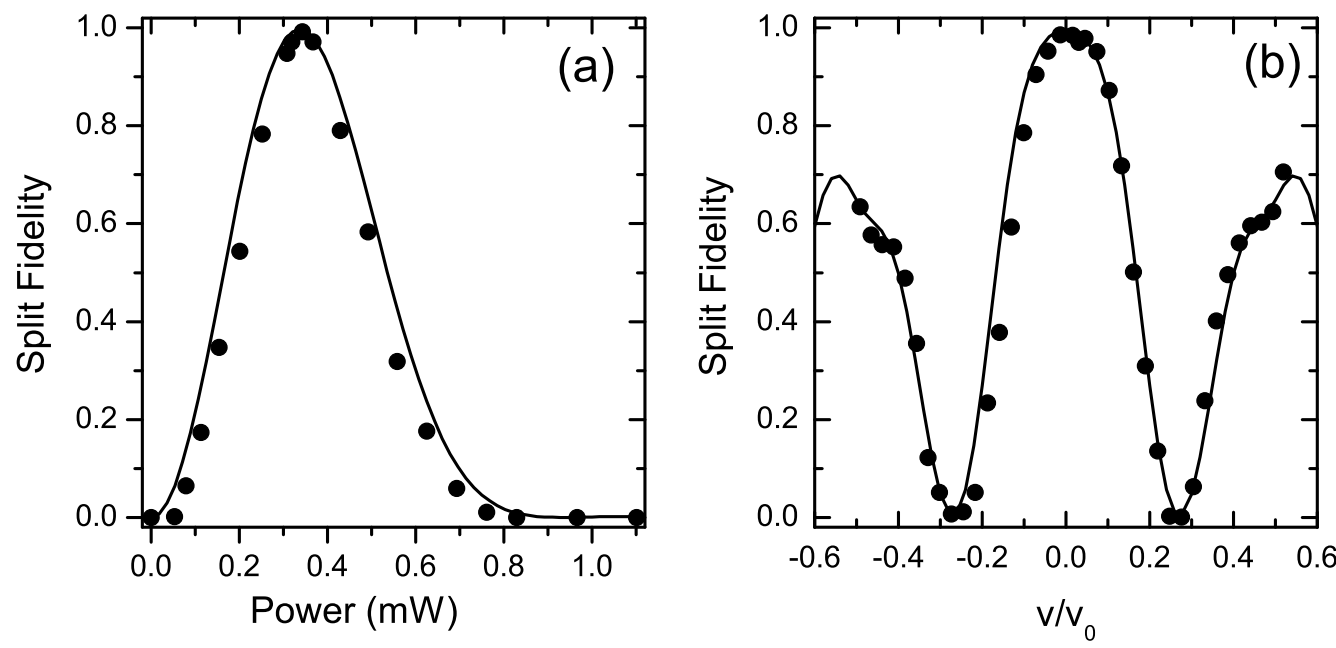

Figure 3.3: Fidelity of the splitting operation. Data points show the experimentally measured fidelity as a function of (a) the standing-wave laser power and (b) initial atomic velocity. The solid curves are theoretical calculations and include a calibration adjustment of 1.19 to relate the standing-wave intensity to the measured power. The pulse sequence consisted of a pulse with duration $24 \mu$ s, a $33 \mu$ s delay, and a second pulse identical to the first. The velocity width observed in (b) corresponds to a temperature of about $60 \mathrm{nK}$, illustrating the benefit of performing the experiment using condensates.

In general, this depends on the phase difference between $c_{+1}$ and $c_{-1}$, which is not observable with imaging. However, the interferometer operation does not depend on this phase as long as it is constant. We can therefore write the fidelity as

$$
F_{\text {split }}=\frac{1}{2}\left(N_{+1}+N_{-1}+2 \sqrt{N_{+1} N_{-1}}\right) .
$$

\subsubsection{Split}

For the splitting operation, the highest fidelity is found experimentally for atoms at rest and a power in the retroreflected standing-wave of $0.34 \mathrm{~mW}$. Fig. 3.3 shows the effects of varying the standing-wave power and initial atomic velocity around these values. Each data point shows one run of the experiment, and repeated measurements show fluctuations of around 0.01 in $N_{0}$. 
The optimum fidelity is found to be above 0.99 , and the experiment was found to exhibit this fidelity over a period of hours before an adjustment of the parameters was necessary.

The theoretical curve was calculated similar to Sec. 3.1.1.2 by numerically solving the Schrödinger equation with the optical potential given by Eq. (3.1) and generalizing the approach in that section to multiple orders using the Bloch expansion

$$
\psi(y, t)=\sum_{n} c_{n}(t) e^{i(2 n k+\kappa) y}
$$

where $\kappa=M v_{i} / \hbar$ [60]. The coefficients $c_{n}$ satisfy the coupled differential equations

$$
i \frac{d c_{n}}{d t}=\frac{\hbar}{2 m}(2 n k+\kappa)^{2}+\frac{\beta}{2}\left(c_{n-1}+c_{n+1}\right)
$$

In the calculation, orders up to $n= \pm 4$ were considered, where the populations in the \pm 4 states was negligible. The initial condition was for all the atoms to be in the $N_{0}$ state (i.e. $c_{0}=1$ ), and the fidelity was calculated as in Eq. (3.40).

To compare the experiment with the calculation, the experimentally measured intensity needs to be related to the standing-wave potential amplitude $\beta$, as described in Sec.3.1.1.2. Doing this, we find the optimal intensity to occur at $\beta_{\text {split }}=2.8 \omega_{r}$ or $P=0.28 \mathrm{~mW}$, which differs from the experimentally measured power by about $20 \%$. This discrepancy can be attributed to experimental errors in the intensity calibration, for example from slight misalignments of the beam relative to the atoms or from deviations of the beam profile from a Gaussian. The calculated curve therefore includes a calibration factor, which is chosen to match the peaks of the experimental and theoretical curves.

We also have the possibility to implement higher order splitting pulses, as described by Wu et al. [60], e.g. $|0\rangle \rightarrow\left(\left|+2 v_{0}\right\rangle+\left|-2 v_{0}\right\rangle\right) / \sqrt{2}$. The predicted parameters and fidelities are given in Table 3.1. The fidelities measured are 0.92 for $n=2$, and 0.8 for $n=3$. The low fidelity for the third order pulse can be attributed to the high sensitivity of the operation to pulse intensity and duration and the limited timing resolution of our AOMs, limited by their $100 \mathrm{~ns}$ switching times. 


\begin{tabular}{ccccc}
\hline \hline & \multicolumn{4}{c}{$n$} \\
& 1 & 2 & 3 & 4 \\
\hline$\beta / \omega_{r}$ & 2.83 & 13.4 & 33.9 & 57.6 \\
$\omega_{r} \tau_{1} / 2 \pi$ & 0.084 & 0.148 & 0.029 & 0.067 \\
$\omega_{r} \tau_{2} / 2 \pi$ & 0.143 & 0.112 & 0.088 & 0.045 \\
$\omega_{r} \tau_{3} / 2 \pi$ & 0.080 & 0.172 & 0.031 & 0.024 \\
$F$ & 1 & 0.991 & 0.966 & 0.917 \\
\hline \hline
\end{tabular}

Table 3.1: Parameters for higher order splitting operations from Ref. [60] using a numerical calculation. The sequence is the double pulse sequence described in Sec. 3.1.1.2 with pulses of duration $\tau_{1}$ and $\tau_{3}$ separated by a time $\tau_{2}$. F denotes the calculated split fidelity. For our experiment, the calculated values for $n=1$ are $\tau_{1}=22 \mu \mathrm{s}, \tau_{2}=38 \mu \mathrm{s}$, and $\tau_{3}=21 \mu \mathrm{s}$, in good agreement with the values obtained with the analytical calculation.

The fourth order pulse was not implemented due to the fact that the required laser power was more than the power that was available.

\subsubsection{Reflect}

A similar measurement as in the previous section was performed for the reflection operation, as seen in Fig. 3.4. Here, the desired end state is $N_{+2}$, since the initial velocity is close to $-v_{0}$. The measured maximum fidelity in this case was 0.94 , at a power of $0.5 \mathrm{~mW}$. Again, a calibration factor was used, and the ratio of powers of the standing-wave for the splitting and reflection operations was found to be 0.68 , in good agreement with the theoretical value for the amplitude of the standing-wave potential $\beta_{\text {reflect }}=4.4 \omega_{r}$.

As seen in Fig. 3.4 the maximum reflection fidelity is lower than the maximum splitting fidelity. This can be improved by using a longer and less intense reflection pulse. In Fig. 3.5, the pulse length was varied and $\beta$ varied to numerically optimize the fidelity at each point to give the theoretical curve. The second maximum was experimentally found at a pulse time of $t=140 \mu \mathrm{s}$ and $\beta=3.0 \omega_{r}$. A maximum fidelity of 0.98 was found for these parameters. By varying the initial velocity of the atoms as in Fig. 3.4, it can be seen that this higher fidelity reflection operation has an increased sensitivity to velocity. Therefore, the shorter pulse may be preferable when the 

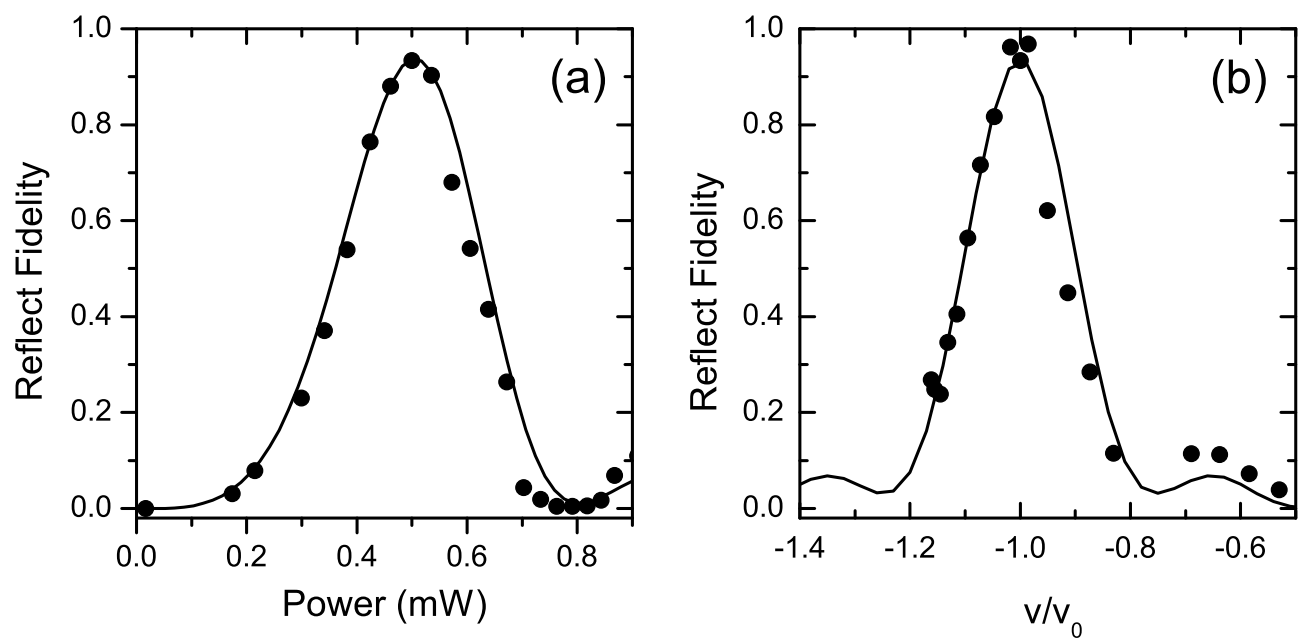

Figure 3.4: Fidelity of the reflection operation. Data points show the experimentally measured fidelity and solid curves are theoretical calculations. The operation consists of a single pulse with duration $76 \mu \mathrm{s}$.

initial velocity cannot be perfectly controlled.

\subsection{Experimental realization}

With working splitting, reflection, and recombination operations, we are ready to implement an interferometer with our device. The schematic of the actual interferometer sequence can be seen in Fig. 3.6. At $t=0$, we apply the splitting operation to transfer the atoms at rest into two packets moving apart along the waveguide axis with velocity $v_{0}$. The packets move apart for a time $T / 4$, where $T$ is the total time in the interferometer, and then the reflection operation is applied, i.e. the velocity of the packets is changed $v_{0} \leftrightarrow-v_{0}$. The atoms then propagate for a time $T / 2$, after which another reflection pulse is applied. The atoms finally propagate for $T / 4$ before the splitting pulse is applied again to recombine the packets. Two reflection operations are performed so that the two packets of atoms traverse nearly the same path and experience the same phase shifts from the guide potential. On the one hand, this cancels out any phase shifts from asymmetries in the guide potential or external field gradients, on the other hand, 

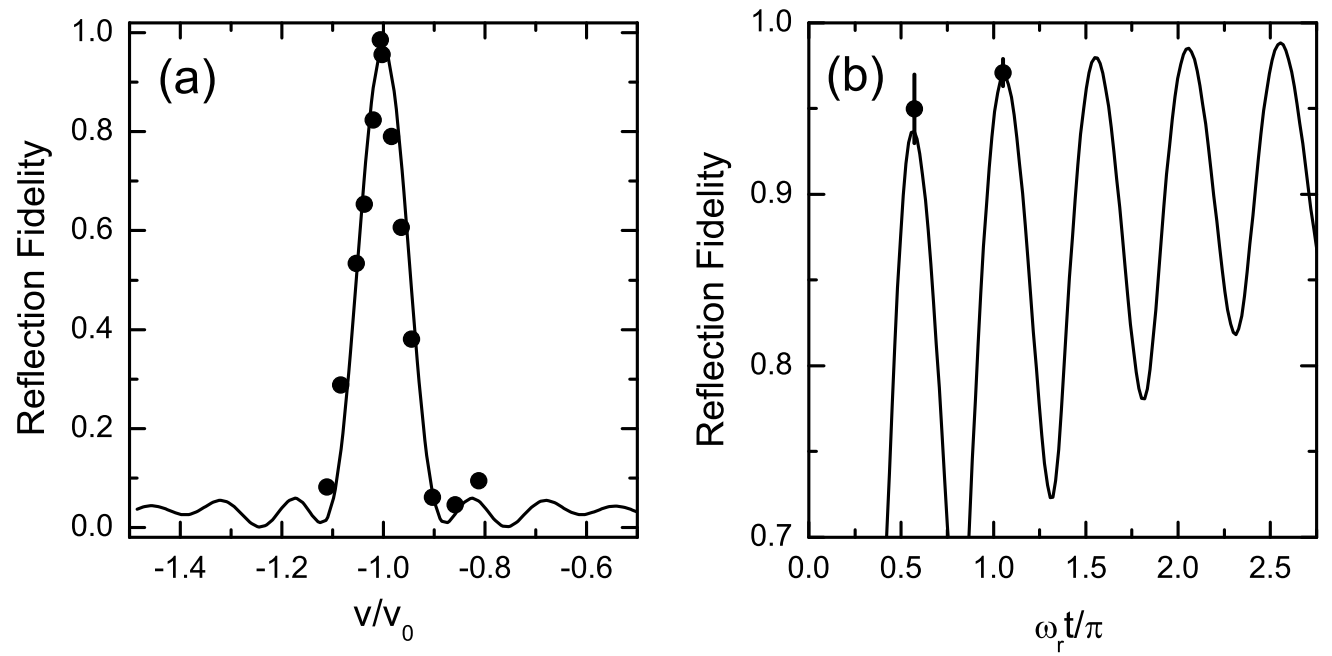

Figure 3.5: (a) Experimental results for a longer reflection pulse. Here $\omega_{r} t=3.3$ and the beam power is $0.37 \mathrm{~mW}$. (b) Optimized reflection fidelity vs. pulse duration. For each pulse duration $t$, the standing-wave amplitude $\beta$ was adjusted to provide the best reflection fidelity for atoms traveling at $v_{i}=v_{0}$. Data points show the experimental results of Fig. 3.4 and part (a) above. Error bars indicate the standard deviation of several measurements.

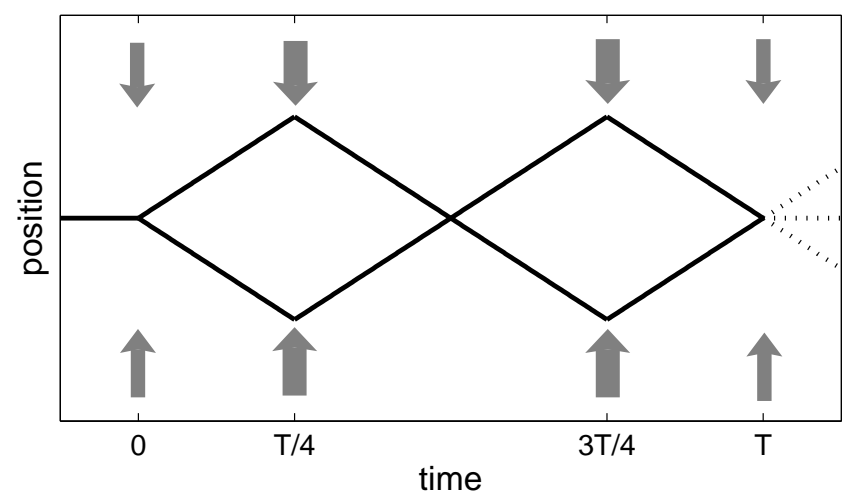

Figure 3.6: Trajectory of wavepackets in the interferometer. The condensate atoms begin nominally at rest. At $t=0$, an off-resonant laser beam (indicated by the arrows) splits the condensate into two packets traveling at $v_{0}= \pm 1.2 \mathrm{~cm} / \mathrm{s}$. At times $T / 4$ and $3 T / 4$, the laser is used to reverse the atoms' motion. At time $T$, a recombination pulse brings the atoms back to rest with a probability that depends on the interferometer phase. The moving atoms (if any) continue to propagate until the system is imaged to determine the output state. 
it also cancels out most of the phase gradients the packets acquire during the first half of the interferometer, as will be described in Sec. 3.3.3.

The geometry of this configuration most closely resembles a Michelson interferometer [17]. This geometry is most suited to the one-dimensional waveguide we use in our experiments and is relatively simple to implement. However, a Michelson interferometer does not enclose any area between the two arms, and so measurements of the Sagnac effect $[25,26]$ are not immediately possible with this device. If measuring rotations is desired, a possibility would be to oscillate the atoms in a direction perpendicular to the guide axis and apply the splitting and reflection operations at points suitable to enclosing an area. Another possibility would be to design a new waveguide in which the minimum of the waveguide is no longer linear, but a circle. These ideas might be implemented in the future, but a range of interesting measurements are already possible in the linear guide which we have at our disposal.

To measure the output of the interferometer, we let the packets of atoms separate for some time (usually around $40 \mathrm{~ms}$ ) after recombination. As given by Eq. (3.35), we expect the fraction of atoms at rest to depend on the differential phase $\phi$ between the two packets before recombination,

$$
\frac{N_{0}}{N}=\cos ^{2}\left(\frac{\phi}{2}-\alpha\right)
$$

We can measure this fraction by taking an absorption image of the three packets and by fitting each packet to a two-dimensional Gaussian function. The number of atoms in each packet can then be extracted by the procedure given in Sec. 2.1.4. While strictly speaking the density profile of the clouds of condensate atoms is more closely given by a Thomas-Fermi function [4], a Gaussian fit is a good approximation and is easier to implement. Furthermore, we measure ratios, so any systematic error in the absolute atom number in a packet from using the Gaussian fit function should cancel when taking the ratios $N_{0} / N$.

For the first experiments, we do not want the atoms to acquire a differential phase $\phi$ from experiencing different environments. This type of experiment would likely need some calibration, as the change of environment would come from an external field $[53,64]$. However, we still 


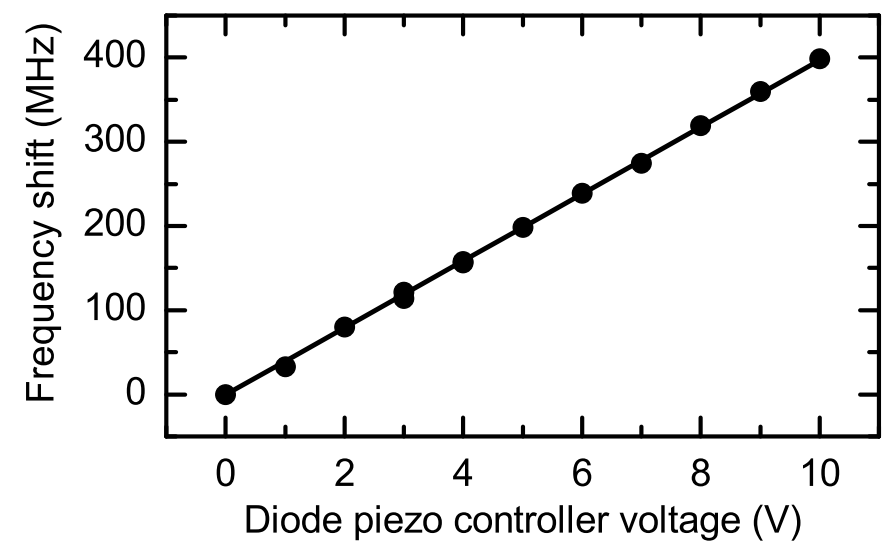

Figure 3.7: Calibration of the frequency shift of the standing-wave laser beam generated by a homebuilt diode laser. For a voltage applied to the grating piezo controller of the diode laser used to generate the standing wave, the frequency shift was measured. The frequency shift is seen to be proportional to the applied voltage.

want to observe the interferometer operation by varying the fraction of atoms that remain at rest $N_{0} / N$. As described in Sec. 3.1.1.4, we can do this by changing the phase of the standing wave $\alpha$ relative to the atoms between splitting and recombination pulses. From Eq. (3.30), we know that $\alpha=-2 k D$, where $k$ is the wavenumber of the standing wave and $D$ is the mirror position. A change in $\alpha$ can therefore be caused by a change of mirror position or a change in the wavenumber, i.e. a change in the frequency. Since the mirror position is fixed in our experiment a distance $D=22.5 \mathrm{~cm}$ away from the atoms, we shift the frequency to change the phase of the standing wave. The phase shift can then be expressed in terms of the frequency shift $\Delta v$ as

$$
\alpha=\frac{4 \pi D}{c} \Delta v
$$

A phase shift of $\pi / 2$, where no atoms remain at rest is therefore expected for a detuning shift of $167 \mathrm{MHz}$. In the experiment, the frequency is shifted by slightly changing the grating position of the diode laser used to generate the standing wave. The change of frequency takes about $2 \mathrm{~ms}$ to complete, and is approximately proportional to the applied change in voltage to the piezo controlling the grating position, as seen in Fig. 3.7 


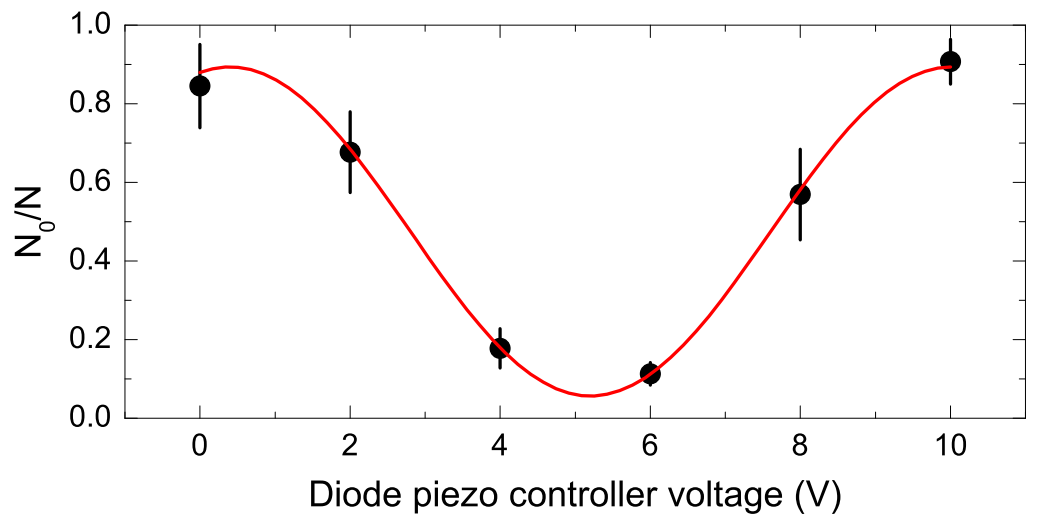

Figure 3.8: Interference curve for $T=40 \mathrm{~ms}$, taken by measuring the fraction of atoms that remain at rest after recombination $N_{0} / N$. For each value of the piezo controller voltage, which is proportional to the standing wave phase $\alpha$, several images were obtained and averaged to get $N_{0} / N$, where the error bars denote the standard deviation of the mean. The solid curve is a fit of the data.

For the following experiments, the waveguide frequencies were always $\left(\omega_{x}, \omega_{y}, \omega_{z}\right)=2 \pi \times$ $(6,1.2,3.3) \mathrm{Hz}$, and the waveguide was loaded with 2 to $3 \times 10^{4}$ atoms. The Bragg beam was detuned $7.8 \mathrm{GHz}$ blue of the $5 S_{1 / 2} \rightarrow 5 P_{3 / 2}$ transition for the measurement from 2006 , and $12.8 \mathrm{GHz}$ blue for the other, more recent measurements.

\subsubsection{Visibility curve}

By measuring the fraction of atoms that remain at rest for various standing-wave phases, an interference curve is generated, as seen in Fig. 3.9(a). This curve can be fit to a function of the form

$$
f(s)=y_{0}+A\left[\cos \left(2 \pi \frac{s-s_{0}}{P}\right)\right]
$$

where $y_{0}$ is the average value, expected to be close to $0.5, A$ is the amplitude, $P$ is the fit period, and $s_{0}$ is an offset. Two important parameters can be extracted from this fit: the visibility $V$ and the phase shift $\theta$.

The visibility describes the contrast of the interferometer and is therefore a measure of how 
good the coherence in the interferometer is preserved. In general, the visibility of an interference pattern with maximum value $I_{\max }$ and minimum value $I_{\min }$ is defined as [17]

$$
V=\frac{I_{\max }-I_{\min }}{I_{\max }+I_{\min }}
$$

For the fit function from Eq. (3.45), the visibility is therefore given by

$$
V=\frac{A}{y_{0}}
$$

The phase shift $\theta$ is a measure of the differential phase acquired by the two arms of the interferometer before recombination. For the case of no applied differential phase shift, we expect its value to be zero. However, we observe a scatter of this phase shift around zero, due to potential fluctuations and imperfections in fitting the data. From the fit, the phase shift can be extracted as

$$
\theta=\frac{2 \pi s_{0}}{P}
$$

The first measurement (from 2006) of the visibility for various total times $T$ of the interferometer can be seen in Fig. 3.9(b). As seen in the graph, the visibility is around 0.5 for short times and then falls off for times longer than $44 \mathrm{~ms}$. At the time, this was four times longer than the previous longest coherence time for a condensate interferometer observed by Wang et al. [53]. However, shortly afterwards, Jo et al. demonstrated a coherence time of $200 \mathrm{~ms}$ using a different interferometer scheme [65].

\subsubsection{Improvements}

After measuring this first visibility curve, various improvements were performed on the experiment which increased the visibility at short times and also increased the coherence time. 


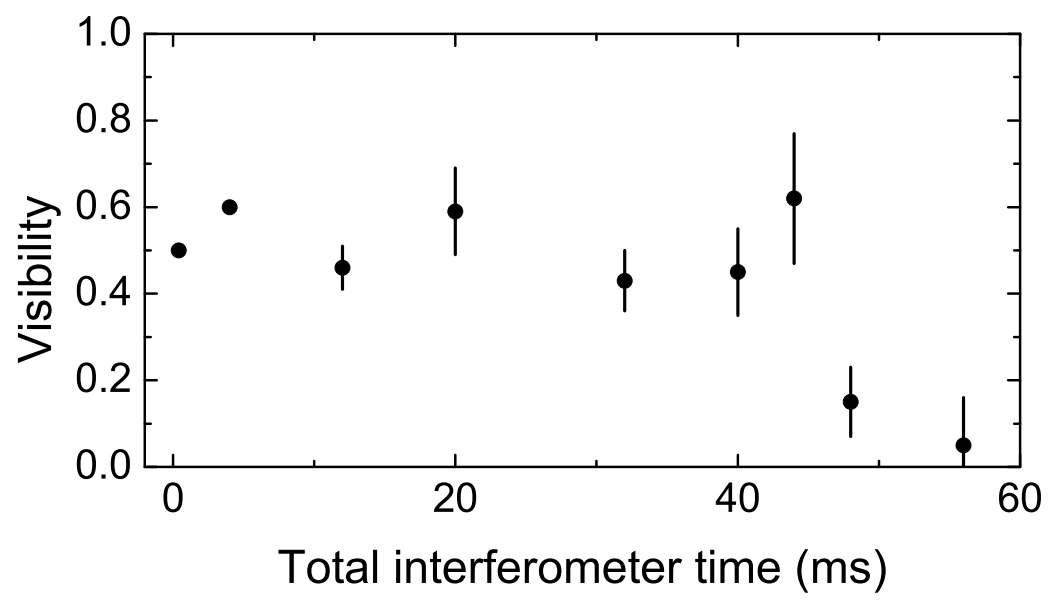

Figure 3.9: Visibility data from 2006 for the interferometer before the improvements described in Sec.3.2.2 were implemented. For a range of times, interference curves such as in Fig. 3.8were obtained and fit to obtain the visibility. At shorter times, it was not possible to change the laser wavelength fast enough, so a set of points at $\alpha=0$ only were used to estimate the visibility as $V \approx 2\left\langle N_{0} / N\right\rangle-1$.
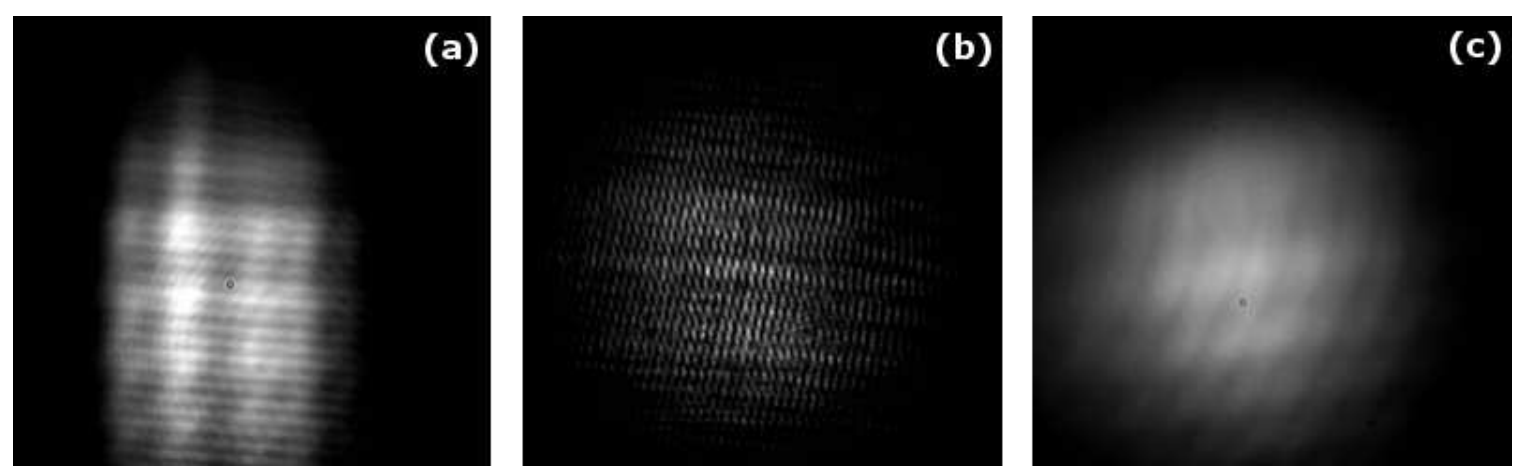

Figure 3.10: Images of the beam used to generate the standing wave (a) after passing through the glass vacuum chamber once and (b) after being retroreflected. The spatial interference pattern comes from etaloning from the glass surfaces of the chamber, which were not anti-reflection coated. (c) Image of the beam after retroreflection using a chamber with anti-reflection coated windows. The interference pattern is greatly reduced. 
Beam quality After the initial success in implementing the interferometer, the efficiency of the splitting and reflection operations decreased and were inconsistent from one run of the experiment to the next. Looking at the Bragg beam after passing through the vacuum chamber and being retroreflected, we saw a strong spatial interference pattern on the beam, as seen in Fig. 3.10 , This interference pattern comes from etaloning from the glass-air and glass-vacuum surfaces of the chamber, which were not anti-reflection coated. While the reflection from a single surface is only $4 \%$ [17], considering all possible reflections is enough to explain the striking interference pattern. In a spatially varying intensity pattern such as this one, the efficiency of the splitting and reflection operations is expected to vary as atoms are in different positions relative to the pattern, either due to slightly different positions of the atoms, or from a shift in position of the interference pattern. After replacing the chamber with a new one with anti-reflection coated windows, a great improvement in beam quality and also consistency of splitting and reflection operations was observed.

Atomic motion Another detriment to a well-working interferometer is residual atomic motion in the waveguide. As seen in Sec. 3.1.2.1 any velocity of the atoms at the time of the splitting pulse will decrease the fidelity of the operation, as well as the fidelities of the subsequent reflection and recombination operations. The visibility of the output will therefore be reduced. We found that most of the residual motion we observed came from loading the waveguide even after using the loading sequence described in Sec.2.2.4. During the loading sequence, the trap frequencies pass through $60 \mathrm{~Hz}$ for some value of the external and waveguide fields. At this point, noise from the power line at $60 \mathrm{~Hz}$ drives the atoms and they start oscillating. This oscillation continues with increased amplitude as the confinement frequency is further reduced.

Two possibilities exist for alleviating this problem. We could ramp the external and waveguide fields to such values that the confining frequencies are below $60 \mathrm{~Hz}$ in all directions during the final step of evaporation. Since the motion of the cloud looks like heating in the frame of the trap, evaporative cooling will then restrict the motion of the atoms. However, since the evapora- 


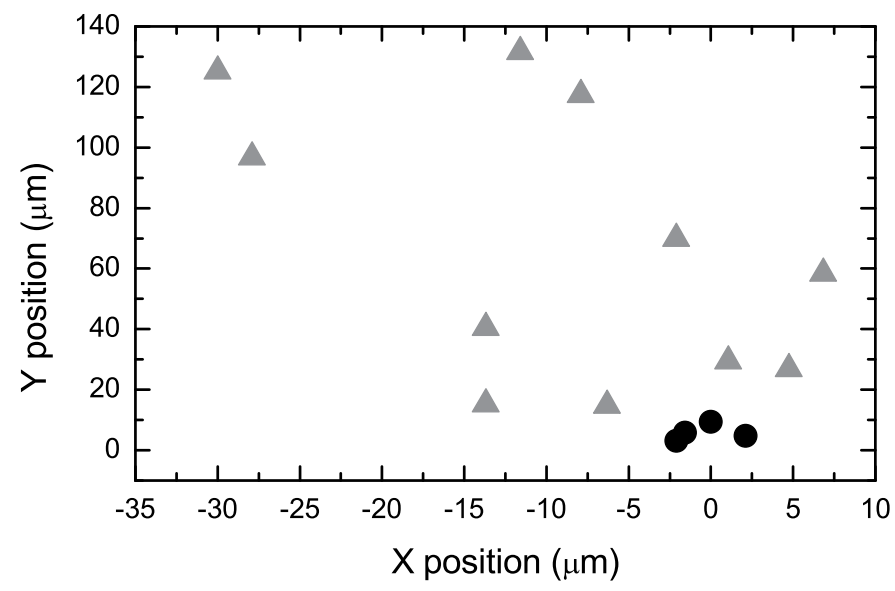

Figure 3.11: Position of the atoms in the waveguide for several runs of the experiment. Gray triangles show the spread of positions without a synchronization, black circles show the great improvement when synchronizing the waveguide loading procedure to $60 \mathrm{~Hz}$.

tion is performed in a weaker trap, the collision rate is reduced and so evaporation will proceed at a slower pace than in the stronger trap. We initially got such a sequence to work by ramping down the external quadrupole to $B_{x}^{\prime}=135 \mathrm{G} / \mathrm{cm}$ after loading the atoms into the TOP trap and then performing a longer evaporation. The largest TOP trap frequency in this configuration was $\omega_{z}=2 \pi \times 54 \mathrm{~Hz}$. The number of atoms was somewhat lower in this case. The next time we tried using this technique, we performed the first step of evaporation after loading the atoms into the TOP trap in a $155 \mathrm{G} / \mathrm{cm}$ external quadrupole and $4 \mathrm{G}$ waveguide bias field trap $\left(\omega_{z}=2 \pi \times 136 \mathrm{~Hz}\right)$. Before the final step of evaporation, either the external quadrupole was ramped to $58 \mathrm{G} / \mathrm{cm}\left(\omega_{z}=2 \pi \times 52 \mathrm{~Hz}\right)$ or the waveguide bias field was ramped to $20.5 \mathrm{G}$ with the external quadrupole at $135 \mathrm{G} / \mathrm{cm}\left(\omega_{z}=2 \pi \times 54 \mathrm{~Hz}\right)$. In this implementation, the number of atoms in the condensate was still somewhat reduced, but the oscillation of the atoms was not sufficiently suppressed.

Another possibility to minimize atomic motion is to synchronize the experiment to the $60 \mathrm{~Hz}$ given by the line voltage. This means that the atoms are still excited and oscillate, but that we access the same position of the oscillation every run of the experiment. We can then perform 
the interferometer operations at an extremum of the oscillation, where the packet velocity is near zero and splitting and reflection operations are consistently efficient. The effect of this synchronization can be seen in Fig. 3.11 .

Imaging An improvement of the imaging was achieved by upgrading the camera used for the absorption imaging to a model with a faster download time. As explained in Section 2.1.4 when imaging the atoms, we follow the picture of the atoms with a picture with the same settings, but without the atoms. The new camera allowed the time between taking the "atoms" and "no atoms" images to be reduced from $20 \mathrm{~s}$ to $2 \mathrm{~s}$, which significantly reduced the noise on the image.

Trap noise One of the most important features in our experiment is the waveguide. The stability of the fields in the waveguide is paramount to a successful interferometer operation, as fluctuations immediately translate into fluctuations of the phase of the atoms. The trap stability is controlled by feedback, as explained in Ref. [48]. However, the stability reported in Ref. [48] was measured for an isolated system. In the working interferometer, the amplitude of the waveguide fields is controlled by varying the voltage to the amplitude modulation input of the function generators used in the waveguide circuit. We found that this increased the noise floor on the waveguide bias fields from $-120 \mathrm{dBV} / \sqrt{\mathrm{Hz}}$ with the amplitude modulation off to $-90 \mathrm{dBV} / \sqrt{\mathrm{Hz}}$ with it on. We therefore built a circuit for amplitude modulation ourselves using an analog multiplier, which reduced the noise floor to $-105 \mathrm{dBV} / \sqrt{\mathrm{Hz}}$. A further improvement would be expected if we turned the amplitude modulation off after reaching the final value for the waveguide fields, however we found that this introduced a slight phase shift between the bias fields which varied from day to day, so we did not pursue this further.

With these improvements, we again performed the interferometer experiments described above, and measured the interferometer visibility as a function of time in the interferometer $T$, as seen in Fig. 3.12, At short times, we now observe almost perfect visibility, and the coherence time is extended by almost a factor of two to about $72 \mathrm{~ms}$. 


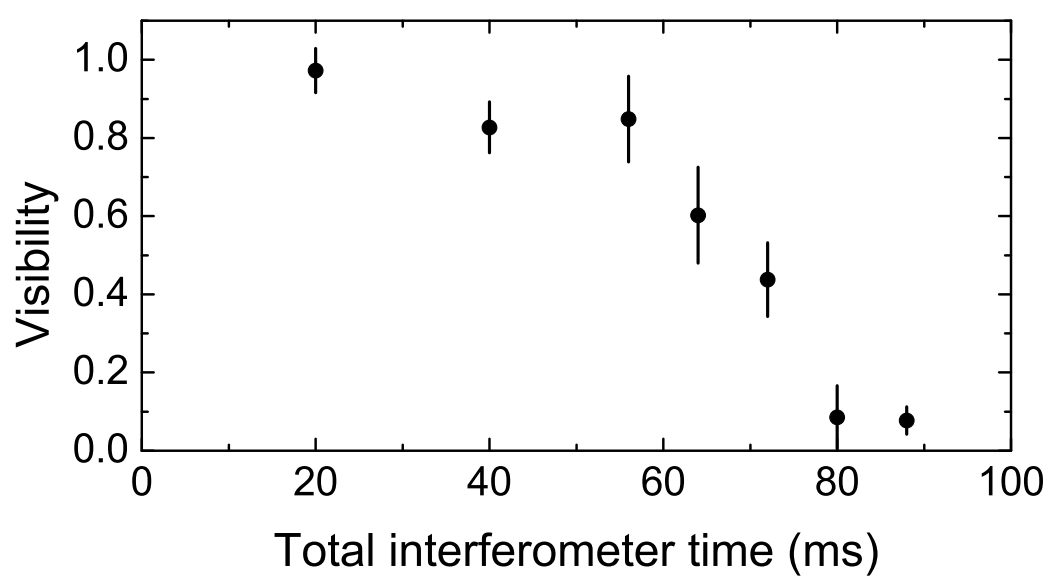

Figure 3.12: Visibility after implementation of the improvements described in the text. The visibility at short times is now almost perfect, and we observe coherence up to times of $72 \mathrm{~ms}$.

\subsubsection{Arm separation}

One of the major features of our interferometer is its unprecedented arm separation. Previously, atomic wave functions have been split over distances of up to $1.1 \mathrm{~mm}[31,66]$, though the atoms in these cases were located in clouds or a beam with a width of several $\mathrm{mm}$. The separate packets were therefore not accessible in the way the arms of a light interferometer are. Of course, some measurements such as gravity or rotations do not require a separation of the packets in the interferometer, and conventional atom interferometers have been highly effective in measuring these effects $[28,31,66,67]$. However, separated arms permit many additional uses. With tightly collimated atomic beams, separated arms were achieved and allowed precise measurements of atomic polarizabilities [44,68], phase shifts in atomic and molecular scattering [69], and atomsurface interactions [70].

With Bose-Einstein condensates, packet displacements of up to $100 \mu \mathrm{m}$ have been achieved $[53,71]$, though in this case the individual packets were even larger, which did not allow for individual access. A separation of distinct packets was achieved by splitting the atoms between two optical traps with a maximum spacing of only $13 \mu \mathrm{m}[64,72]$.

In our experiment, the maximum arm separation at which the atoms are still coherent is 


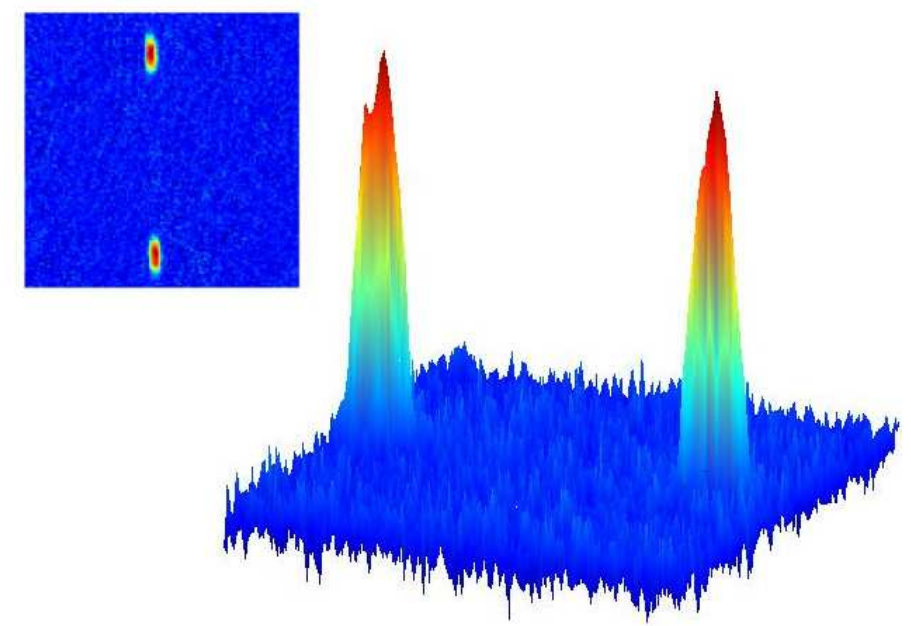

Figure 3.13: Separated packets in the interferometer, shown as both a three-dimensional image and a flat picture. The absorption image was take $18 \mathrm{~ms}$ after the splitting pulse and illustrates the maximum separation for an experiment with $T=72 \mathrm{~ms}$. Since the interference visibility is non-zero for this $T$, the atoms in this picture are in a quantum superposition of being in both peaks. The center-to-center separation of the two packets is $0.42 \mathrm{~mm}$.

found to be $0.42 \mathrm{~mm}$, at an interferometer time $T=72 \mathrm{~ms}$. A representation of this separation can be seen in Fig. 3.13. This separation, which corresponds to the thickness of about 4 sheets of paper, allows for a variety of applications.

One possibility would be to pass one arm into an optical cavity, where it would acquire a phase shift which depends on the field in the cavity. The number of photons in the cavity could then be measured in a non-destructive way, similar to the experiments of Nogues et al. [73]. Another application would be to bounce one arm of the interferometer off a material surface through quantum reflection $[74,75]$. Measuring the phase shift from the reflection would provide information about effects such as the Casimir-Polder force. Experiments such as these would require the atoms to either traverse separate paths, or for the surface to be removed before the second packet arrives, to allow for a differential phase shift between the two arms.

Finally, our interferometer is well-suited for measurements of polarizabilities of the condensate atoms, as will be described in Chapter 4. In the simplest case, we can shine a beam of light onto one packet and not the other, to measure ac polarizabilities. Furthermore, passing the 
packets between the plates of a parallel-plate capacitor and applying a static electric field to one packet allows a precise measurement of the dc polarizability.

\subsection{Limitations}

In the previous section, we saw that we can achieve long interference times up to $72 \mathrm{~ms}$ and large arm separations of almost $0.5 \mathrm{~mm}$ with our interferometer. However, we are still not at a point where our measurements can necessarily be competitive with those performed with thermal beam atom interferometers. In this section, I will describe several effects which limit our coherence times.

\subsubsection{Phase diffusion}

We first consider a fundamental limitation to the interferometer performance. In an interferometer such as ours, the splitting operation is necessarily limited to placing about half of the atoms in either of the $| \pm 2\rangle$ states. This slight asymmetry will cause slightly different self-interaction energies and as a result cause fluctuations in the interferometer output for various runs of the experiment, therefore reducing the visibility.

We can quantify this effect by considering the many-particle wavefunction in terms of the two states $|+2\rangle$ and $|-2\rangle$, which correspond to packets of atoms moving with momenta $\pm 2 \hbar k$. For $N$ total atoms and no acquired phase, the $N$-particle wavefunction is then given by

$$
|\chi\rangle=\left[\frac{1}{\sqrt{2}}(|+2\rangle+|-2\rangle)\right]^{\otimes N},
$$

where $\otimes$ is the Hilbert space product. It is advantageous to write the wavefunction in terms of the number states of the two packets. We denote a state with $j$ atoms in the $|+2\rangle$ state and $N-j$ 
atoms in the $|-2\rangle$ state as $|j, N-j\rangle$. The state $\chi$ can then be expressed as

$$
|\chi\rangle=\frac{1}{2^{N}} \sum_{j=0}^{N} \sqrt{\left(\begin{array}{c}
N \\
j
\end{array}\right)}|j, N-j\rangle
$$

The fact that the binomial coefficient appears with a square root is the consequence of changing to a basis of bosonic number states, which need to be properly symmetrized.

Considering interatomic interaction effects as described in Sec. 1.2, we can now consider phase diffusion. We assume that the energies $E$ of the two packets are the same for same number of particles, i.e. $E_{+2}(j)=E_{-2}(j)=E(j)$. The total energy can then be expanded around the expectation value $j=N / 2$ as

$$
E_{+2}(j)+E_{-2}(N-j) \approx \hbar\left[\omega_{0}+\xi(j-N / 2)^{2}\right]
$$

where

$$
\omega_{0}=\frac{2 E(N / 2)}{\hbar}, \quad \text { and } \quad \xi=\left.\frac{1}{\hbar} \frac{d^{2} E(j)}{d j^{2}}\right|_{j=N / 2} .
$$

For a condensate, the energies are given by $E(j)=\int \mu(j) d j$, where $\mu$ is the chemical potential, and therefore

$$
\xi=\left.\frac{1}{\hbar} \frac{d \mu(j)}{d j}\right|_{j=N / 2} .
$$

The evolution in time of the wavefunction (3.50) then follows

$$
|\chi, t\rangle=\frac{e^{-i \omega_{0} t}}{2^{N / 2}} \sum_{j=0}^{N} \sqrt{\left(\begin{array}{c}
N \\
j
\end{array}\right)} e^{-i \xi t(j-N / 2)^{2}}|j, N-j\rangle,
$$

which shows a scrambling of phases and therefore possible phase diffusion. Since we are measuring a phase $\eta$, from quantum mechanics we would expect to be measuring the value of a hermitian phase operator $\hat{\eta}$. Though many attempts at constructing phase operators have been attempted, the construction of such a phase operator is impossible [76]. However, an operator 
for the $\{|+2\rangle,|-2\rangle$ \} states can be usefully approximated, which has eigenstates

$$
|\eta\rangle=\frac{1}{\sqrt{N-1}} \sum_{j=0}^{N} e^{i j \eta}|j, N-j\rangle,
$$

with corresponding eigenvalues $\eta=2 \pi p /(N+1)$ for $p=-N / 2,-N / 2+1, \ldots, N / 2$.

For large $N$, a binomial distribution can be approximated by a Gaussian distribution, and the sum can be turned into an integral. The probability of measuring the many-particle state $|\chi, t\rangle$ to have some phase $\eta$ can now be calculated to be

$$
P(\eta)=|\langle\eta \mid \chi, t\rangle|^{2}=\sqrt{\frac{\pi}{2(\Delta \eta)^{2}}} \exp \left[-\frac{\eta^{2}}{2(\Delta \eta)^{2}}\right],
$$

where the width of the phase distribution is given by

$$
\Delta \eta=\sqrt{\frac{1}{N}+N \xi^{2} t^{2}}
$$

This means that we start with a width of $\Delta \eta=1 / \sqrt{N}$ initially. The width of the phase distribution then increases linearly in time with a rate $R=\sqrt{N} \xi$. We note that this diffusion process is linear in $t$, as opposed to most other diffusion processes which evolve as $\sqrt{t}$.

Javanainen and Wilkens perform a complete calculation of the phase diffusion rate in the Thomas-Fermi approximation and find [77]

$$
R=\left(\frac{72}{125}\right)^{1 / 5}\left(\frac{a}{a_{\mathrm{ho}}}\right)^{2 / 5} \frac{\omega_{\mathrm{ho}}}{N^{1 / 10}}
$$

where $\omega_{\mathrm{ho}}$ is the harmonic mean of the trapping frequencies of the potential and $a_{\mathrm{ho}}=\sqrt{\hbar / m \omega_{\mathrm{ho}}}$ is the characteristic harmonic oscillator length. While this calculation was performed for a splitting routine in which a delta-function potential barrier is erected to separate a harmonic potential into two sides, it gives us a good order of magnitude estimate for the effect of phase diffusion in our system. For the weakest confinement in our trap, we find $R \approx 0.35 \mathrm{~s}^{-1}$. It follows that the phase diffusion time is then approximately $\tau_{r} \approx 3 \mathrm{~s}$, or much longer than the interferometer 
times we realize in the presented experiments.

\subsubsection{Atomic interactions}

In addition to interactions within the packets, there is also an interaction phase between the two packets acquired whenever parts of the packets overlap. This effect is therefore most important at short interferometer times when the packets overlap for a large part of the interferometer sequence. To estimate the effect of these, we treat the interferometer as a one-dimensional system. We are not truly in the one-dimensional regime, but this provides a convenient approximation for what is a small effect. In this regime, we can write the one-dimensional GPE as

$$
i \hbar \frac{\partial}{\partial t} \Psi(y, t)=\left(-\frac{\hbar^{2}}{2 m} \frac{\partial^{2}}{\partial y^{2}}+U_{\mathrm{ext}}(\mathbf{r})+g_{1 \mathrm{D}}|\Psi(y, t)|^{2}\right) \Psi(y, t)
$$

with the one-dimensional coupling constant $g_{1 \mathrm{D}}[78]$.

In the Thomas-Fermi approximation, the one-dimensional density profile $n_{1 \mathrm{D}}$ is given by

$$
n_{1 \mathrm{D}}=\frac{\mu}{g_{1 \mathrm{D}}}\left(1-\frac{y^{2}}{L^{2}}\right) H(L-|y|)
$$

where $\mu$ is the chemical potential of the condensate, $L$ the half-width of the packets, and $H(x)$ is the Heaviside function. From the normalization condition $\int n_{1 \mathrm{D}} d y=N$, we find

$$
\frac{\mu}{g_{1 \mathrm{D}}}=\frac{3 N}{4 L}
$$

The interaction potential between the packets is given by $U_{\mathrm{int}}=2 g_{1 \mathrm{D}} n_{1 \mathrm{D}}$, where the factor of 2 comes from the exchange effect, assuming a delta function interaction potential, as in Eq. (1.10) [79]. However, after splitting, the number density is reduced by a factor of 2, leaving

$$
U_{\mathrm{int}}\left(\xi_{+}\right)=\mu\left(1-\frac{\xi_{-}^{2}}{L^{2}}\right) H\left(L-\left|\xi_{-}\right|\right)
$$


where $\xi_{ \pm}$are the coordinates within the two packets relative to the packet centers.

For short times, we can assume the velocity of the packets is constant, and the centers move apart on trajectories $y_{ \pm}(t)= \pm v_{0} t$, so that the position in one packet $\xi_{+}$coincides with the position $\xi_{-}-2 v_{0} t$ in the other packet. The phase difference due to interactions is then

$$
\phi_{\text {int }}(\xi)=\frac{1}{\hbar} \int_{0}^{T}\left[U_{\text {int }}\left(\xi-2 v_{0} t\right)-U_{\text {int }}\left(\xi+2 v_{0} t\right)\right] d t
$$

The phase gradient at the center of the cloud can then be written as

$$
\frac{d \phi_{\text {int }}}{d \xi}=\left\{\begin{array}{lll}
-8 k\left(\frac{\omega T}{2}\right)^{2} & \text { for } & T / 2<L / 2 v_{0} \equiv t_{s} \\
-8 k\left(\omega t_{s}\right)^{2} & \text { for } & T / 2>t_{s}
\end{array}\right.
$$

where $L$ is the length of our condensate. In our experiment, the centers of the packets separate at a time $t_{s} \approx 2.3 \mathrm{~ms}$. For longer times $\mathrm{T} / 2$, the interaction gradient is a constant at $-1.1 \mathrm{rad} / L$.

\subsubsection{Phase gradients}

The most important limitation of our experiment is the effect of the confining potential. Ideally, a waveguide has no confinement along the waveguide axis. However, as seen in Sec.2.2.3, due to the finite size of our waveguide structure, the potential has the form of an elongated harmonic trap, with a small amount of harmonic confinement along the waveguide axis. In our case, the trapping frequency in this direction ranges from $0.8-1.2 \mathrm{~Hz}$.

It can easily be seen that a nonuniform potential of this sort causes a spatially varying phase shift on the packets as they propagate in the potential. At recombination, different parts of the packets will therefore interfere with different phases, causing the visibility of the interference to be decreased. In this section, measurements of this phase gradient effect are shown and a simple theory to explain these effects is developed.

In the following experiments, $3 \times 10^{4}$ atoms in the condensate were held in the waveguide potential with confining frequencies $\left(\omega_{x}, \omega_{y}, \omega_{z}\right)=2 \pi \times(6,1.2,3.3) \mathrm{Hz}$. The atoms were manipu- 

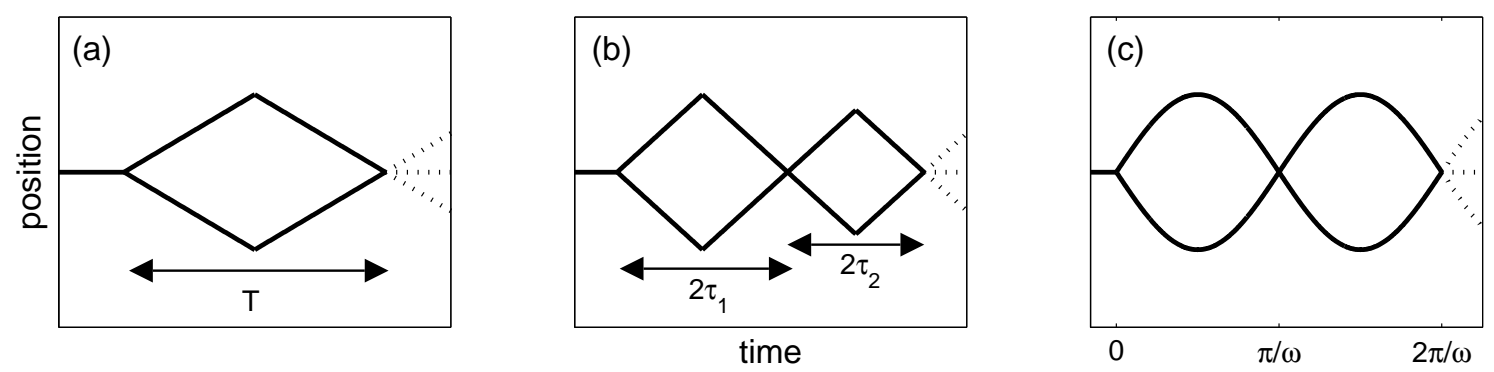

Figure 3.14: Wavepacket trajectories for various interferometer configurations. (a) Single-sided interferometer with leg duration $T / 2$. (b) Double-sided interferometer with leg duration $\tau_{1}$ for the first half of the interferometer and $\tau_{2}$ for the second. (c) Free-oscillation interferometer in a waveguide with harmonic confinement along the guide axis of frequency $\omega$. Note that the time and position scales vary considerably for the different sub-figures.

lated with the standing-wave laser beam, which was detuned $12 \mathrm{GHz}$ blue of the $5 S_{1 / 2}, F=2 \rightarrow$ $5 P_{3 / 2}, F=3$ transition.

We will consider various trajectories of the wavepackets. In Fig. 3.14(a), the simplest case of a single-sided interferometer is shown. In this case, after splitting, the packets are allowed to propagate for a time $T / 2$ before being reflected. After another time $T / 2$, they are recombined and the fraction of atoms at rest $N_{0} / N$ is determined by absorption imaging. After moving apart, the packets move up the confining potential. Since the packets have a finite length $L$ in the waveguide direction, the leading edge of the cloud experiences a different potential than the trailing edge. This means that it accumulates a larger phase, which continues to accumulate throughout its entire trajectory. A spatial gradient of the phase is therefore present on the packets when they are recombined.

The effects of the confining potential were first explored by Olshanii and Dunjko [79] in their interpretation of the results of Wang et al. [53]. Later, Horikoshi and Nakagawa implemented an interferometer with a similar geometry, of which the results obtained were consistent with the interpretation of phase gradients [56]. In both of these experiments however, atomic interactions played a major role. When different parts of the atomic packets interact for different times, as during separation, a phase gradient is also written on the packets. Stickney et al. showed that 
the phase gradients from the interaction and the confinement effects can be made to cancel in some cases, improving the interferometer performance [80].

In our experiment, interactions do not play as big a role as in the experiments described above, due to the relatively weaker confinement. For comparison, in our experiment, the gradient due to interactions at the center of the cloud is given by Eq. 3.64) as $-1.1 \mathrm{rad} / L$. In the experiment of Wang et al., the confining potential had $\omega=2 \pi \times 5 \mathrm{~Hz}$, leading to an interaction gradient of - $14.5 \mathrm{rad} / L$. In addition, our well-separated arms mean that the phase due to interactions stops growing after the arms have separated. Finally, we use a double-sided interferometer, as seen in Fig. 3.14(b) to cancel out the phase gradients in the second half of the interferometer sequence when the two separation times are equal.

We can also imagine achieving larger arm separations by not using any reflection operations, but instead letting the atoms oscillate freely in the confining potential for a full period, as seen in Fig. 3.14(c). In this case the gradient cancels precisely when the packets are aligned properly, but due to the long oscillation period of nearly one second, stability effects start to play a role, and we do not observe phase stability.

\subsubsection{Single-sided interferometer}

To calculate the phase gradients, we develop a simple semi-classical theory. Any internal dynamics of the packets are neglected, since these will occur on a time scale of the external confinement frequency $\omega$. Our experiments are limited by confinement effects to interferometer times $T$ so that $\omega T \ll 1$. We also assume that the atoms start at rest in the minimum of the trap. Adding an initial velocity can change the phase development, but in our experiment, this effect is small enough to neglect.

We now consider the motion of the two packets in a confining harmonic potential of the form

$$
U(y)=\frac{1}{2} m \omega^{2} y^{2},
$$


where $m$ is the atomic mass. The centers of mass of the two packets will be denoted as $y_{ \pm}(t)$, and the coordinates within the cloud relative to the center of mass as $\xi_{ \pm}$. The total phase of a packet accumulated in the interferometer is then

$$
\phi\left(\xi_{ \pm}\right)=\frac{1}{\hbar} \int_{0}^{T} U_{\mathrm{ext}}\left(y_{ \pm}+\xi_{ \pm}\right) d t=\frac{m \omega^{2}}{2 \hbar} \int_{0}^{T}\left[y_{ \pm}(t)+\xi_{ \pm}\right]^{2} d t
$$

After the initial splitting operation in the simple single-sided interferometer, one of the packets follows a trajectory

$$
y_{+}^{\mathrm{I}}(t)=\frac{v_{0}}{\omega} \sin \omega t . \quad(0<t<T / 2)
$$

After propagation for a time $T / 2$, the reflection operation is applied. It is important to note that the reflection operation is not a perfect reflection, but rather applies a momentum kick of $-2 v_{0}$ to the packet of atoms. The trajectory after this operation is therefore

$$
y_{+}^{\mathrm{II}}(t)=\frac{v_{0}}{\omega}\left[\cos \frac{\omega T}{2}-2\right] \sin \left(\omega t-\frac{\omega T}{2}\right)+\frac{v_{0}}{\omega} \sin \frac{\omega T}{2} \cos \left(\omega t-\frac{\omega T}{2}\right) . \quad(T / 2<t<T)
$$

The other packet follows a similar trajectory, but with $v_{0}$ replaced by $-v_{0}$ throughout.

As noted above, the reflection operation is not a true reflection but a 'momentum kick'. This has important consequences, as we will see later. As the packets move to higher potentials, they will slow down. The packet with initial velocity $v_{0}$ will therefore have velocity $v_{0}-\delta v$ with $\delta v \approx\left(\nu_{0} / 2\right)(\omega T / 2)^{2}$ at the time of the reflection operation. Due to the momentum kick, the velocity after the reflection operation will be $-v_{0}-\delta v$, faster than the nominal velocity of $-v_{0}$. The packets then accelerate further when they move back towards the center of the trap and reach velocity $-v_{0}-2 \delta v$ at the nominal time of recombination. The packets are therefore not overlapped precisely at the time $T$. A correction of this effect has been suggested by modifying the length of time between reflection and recombination [80]. For the experimental conditions in our singlesided interferometer, this error is only $0.7 \mu \mathrm{m}$ for $T=20 \mathrm{~ms}$. Since this is much smaller than the packet size of $110 \mu \mathrm{m}$, it can therefore be neglected and we can assume that the centers of the 
packets overlap: $y_{+}(T) \approx y_{-}(T) \approx 0$, so that $\xi_{+} \approx \xi_{-} \approx \xi$.

We can now calculate the phase on one packet of atoms at recombination time $T$ as

$$
\phi_{+}=\frac{m \omega^{2}}{2 \hbar}\left\{\int_{0}^{T / 2}\left[y_{+}^{\mathrm{I}}+\xi_{+}\right]^{2} d t+\int_{0}^{T / 2}\left[y_{+}^{\mathrm{II}}+\xi_{+}\right]^{2} d t\right\}
$$

When taking the difference of the phases of the two clouds, terms with even orders in $v_{0}$ will cancel, since the second cloud has $v_{0} \leftrightarrow-v_{0}$ everywhere. The resulting phase difference is then

$$
\Delta \phi(\xi)=\phi_{+}(\xi)-\phi_{-}(\xi)=4 k \xi\left(2 \cos \frac{\omega T}{2}-\cos \omega T-1\right)
$$

using $v_{0}=2 \hbar k / m$. For short times with $\omega T \ll 1$, we can estimate the phase difference as

$$
\Delta \phi \approx k \xi(\omega T)^{2}
$$

At a time $T=20 \mathrm{~ms}$, the gradient is $8.25 \mathrm{rad} / L$, compared to the gradient from interactions as calculated in the previous section of $-1.1 \mathrm{rad} / L$. The effect of interactions is therefore small, but not negligible.

Coherent state approach A different, but equivalent, approach to getting the phase gradient is to use coherent states [76]. This provides us with a check on the semi-classical calculation. In quantum mechanics, coherent states are the closest quantum states to the classical harmonic oscillator. A Bose-Einstein condensate is simply a collection of atoms which are all in the same quantum state, so a condensate oscillating in a quadratic potential can be described by a spatial distribution of coherent states. While this is an approximation, and ignores atomic interactions as well, it allows us to replicate the semi-classical result with a different technique.

A coherent state $|\alpha\rangle$ in our case has a mean position $y$ and mean momentum $p$ defined by

$$
\alpha=\sqrt{\frac{m \omega}{2 \hbar}}\left(y+\frac{i}{m \omega} p\right) \equiv a+i b .
$$


Any operation performed on the coherent state, such as the splitting and reflection operations, can be modeled by an application of the displacement operator $D(\alpha)=\exp \left[\alpha \hat{\mathbf{a}}^{+}-\alpha^{*} \hat{\mathbf{a}}\right]$. The application of the displacement operator with a purely imaginary component has the effect of imparting a velocity to the coherent state, i.e.

$$
D(i u)|\alpha\rangle=e^{i u \operatorname{Re} \alpha}|\alpha+i u\rangle
$$

which means that the splitting operation on an initial state $\left|\alpha_{1}\right\rangle$ will lead to a state

$$
|\Psi\rangle=\frac{1}{\sqrt{2}}\left(e^{i a u}\left|\alpha_{1}+i u\right\rangle+e^{-i a u}\left|\alpha_{1}-i u\right\rangle\right)
$$

where here $u=v_{0} \sqrt{m / 2 \hbar \omega}$.

The time evolution of a coherent state simply follows $\alpha(t)=\alpha(0) \exp (-i \omega t)$, so letting the packets propagate for a time $\tau=T / 2$ means

$$
|\Psi\rangle=\frac{1}{\sqrt{2}}\left(e^{i a u}\left|e^{-i \omega \tau}\left(\alpha_{1}+i u\right)\right\rangle+e^{-i a u}\left|e^{-i \omega \tau}\left(\alpha_{1}-i u\right)\right\rangle\right)
$$

The reflection operation is then modeled by application of the displacement operator $D(\mp 2 i u)$, after which the state is given by

$$
\left.|\Psi\rangle=\frac{1}{\sqrt{2}}\left(e^{i \phi_{1}} e^{i \phi_{2}}\left|e^{-i \omega \tau}\left(\alpha_{1}+i u\right)-2 i u\right\rangle+\mid \text { packet with }-u\right\rangle\right)
$$

where $\phi_{1}=a u$ and $\phi_{2}=-2 u(a \cos \omega \tau+(b+u) \cos \omega \tau)$. The second packet is the same as the first, but with $u \leftrightarrow-u$ everywhere. After another time evolution for time $\tau$, the state is now

$$
\left.|\Psi\rangle=\frac{1}{\sqrt{2}}\left(e^{i \phi_{1}} e^{i \phi_{2}}\left|e^{-2 i \omega \tau}\left(\alpha_{1}+i u\right)-e^{-i \omega \tau} 2 i u\right\rangle+\mid \text { packet with }-u\right\rangle\right)
$$

The recombination pulse means another application of the displacement operator $D( \pm i u)$. We will consider only those packets with velocities near zero, as those will give us $N_{0} / N$. The fraction 
of the atoms with these velocities are given by the state

$$
\left.\left|\Psi_{0}\right\rangle=\frac{1}{2}\left(e^{i \phi_{1}} e^{i \phi_{2}} e^{i \phi_{3}}\left|e^{-2 i \omega \tau}\left(\alpha_{1}+i u\right)-e^{-i \omega \tau} 2 i u+i u\right\rangle+\mid \text { packet with }-u\right\rangle\right)
$$

with $\phi_{3}=u(a \cos 2 \omega \tau+(b+u) \sin 2 \omega \tau-2 u \sin \omega \tau)$. The total phase of the packet is then given by

$\phi_{+}=\phi_{1}+\phi_{2}+\phi_{3}=u[a(1-2 \cos \omega \tau+2 \cos \omega \tau)+b(-2 \sin \omega \tau+2 \sin \omega \tau)+u(-4 \sin \omega \tau+\sin 2 \omega \tau)]$,

and the total state can be written as

$$
\left|\Psi_{0}\right\rangle=\frac{1}{2}\left(e^{i \phi_{+}}|p+r\rangle+e^{i \phi_{-}}|p-r\rangle\right)
$$

where $p=\exp (-2 i \omega \tau) \alpha_{1}$ and $r=i u\left(e^{-2 i \omega \tau}-2 e^{-i \omega \tau}+1\right)$. The phase $\phi_{-}$is the same as $\phi_{+}$, but with $u \leftrightarrow-u$ everywhere. The fraction of atoms that comes back to rest after recombination is then given by $N_{0} / N=\left\langle\Psi_{0} \mid \Psi_{0}\right\rangle$. In general for two coherent states $|\alpha\rangle$ and $|\beta\rangle$, the inner product is given by $\langle\alpha \mid \beta\rangle=\exp \left(-\frac{1}{2}|\beta-\alpha|^{2}\right) \exp \left(i \operatorname{Im} \beta^{*} \alpha\right)$, where the $|\beta-\alpha|^{2}$ term gives the imperfect overlap between the coherent states [76]. In our case, we can assume perfect overlap. The fraction of atoms that comes back to rest is then

$$
\frac{N_{0}}{N}=\frac{1}{4}\left[2+2 \cos \left(\phi_{+}-\phi_{-}+2 \operatorname{Im} p^{*} r\right)\right]
$$

The total phase is therefore given by

$$
\begin{aligned}
\phi & =\phi_{+}-\phi_{-}+2 \operatorname{Im} p^{*} r \\
& =4 u[a(1-2 \cos \omega \tau+\cos 2 \omega \tau)+b(\sin 2 \omega \tau-2 \sin \omega \tau)] \\
& =4 k\left[y(1-2 \cos \omega \tau+\cos 2 \omega \tau)+\frac{v}{\omega}(\sin 2 \omega \tau-2 \sin \omega \tau)\right] .
\end{aligned}
$$

This is the same result as Eq. (3.70) for $y=\xi$, this time including the effects of an initial velocity. 


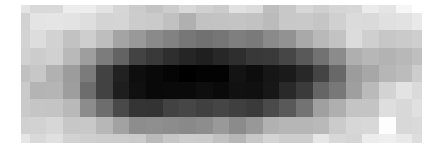

(a)

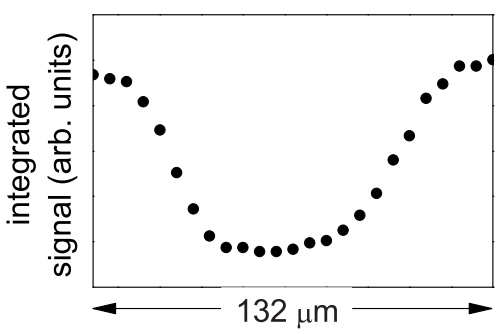

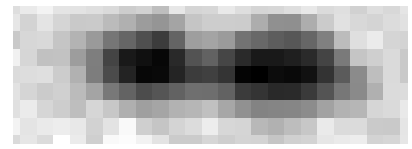

(b)

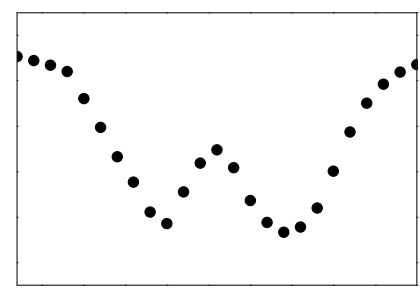

position along waveguide

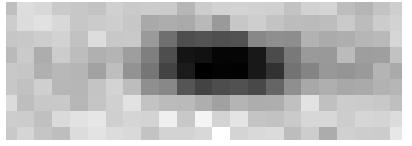

(c)

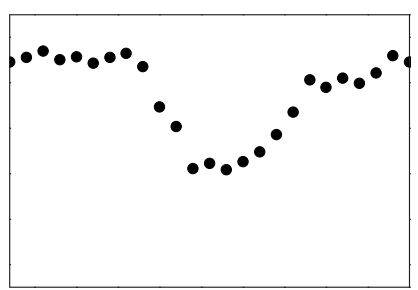

Figure 3.15: Absorption images of wavepackets in the interferometer. (a) The condensate distribution before the interferometer operation. (b) A packet obtained at the output of the interferometer, showing the spatial modulation from the effect of a phase gradient. (c) Another output packet, showing the effect of shifting the overall phase of the interferometer by $\pi$.

Experimentally, the fraction of atoms that remains at rest after recombination is given by $[1+$ $\cos (\theta+\phi)] / 2$, where $\theta$ is an overall applied phase, and $\phi$ is the acquired phase from interactions and confinement effects. Since the phase $\phi$ depends on the position within the packets $\xi$, the spatial density profile after recombination will be modulated. We can observe this modulation by taking absorption images of the clouds after recombination, as in Fig.3.15. Fig.3.15(a) shows the image of a cloud in the waveguide before any interferometer operations, and therefore without any phase gradients. Figs. 3.15(b) and (c) show two packets after recombination, with the phase differing by $\pi$. The spatial modulation of the density profile is clearly seen.

To analyze the effects of phase gradients, we integrate the absorption images in the direction transverse to the interferometer axis, thus gaining a one-dimensional density profile as seen in the figure. This profile is then fit to a modulated Thomas-Fermi profile. We fit 4 images of 3 packets each with a common gradient $G$ and fixed length $L$ to a function

$$
A(y)=A_{0}+B \cos ^{2}\left[\frac{G\left(y-y_{0}\right)+\theta}{2}\right] \cdot \min \left[1-\left(\frac{y-y_{0}}{L}\right)^{2}, 0\right]
$$



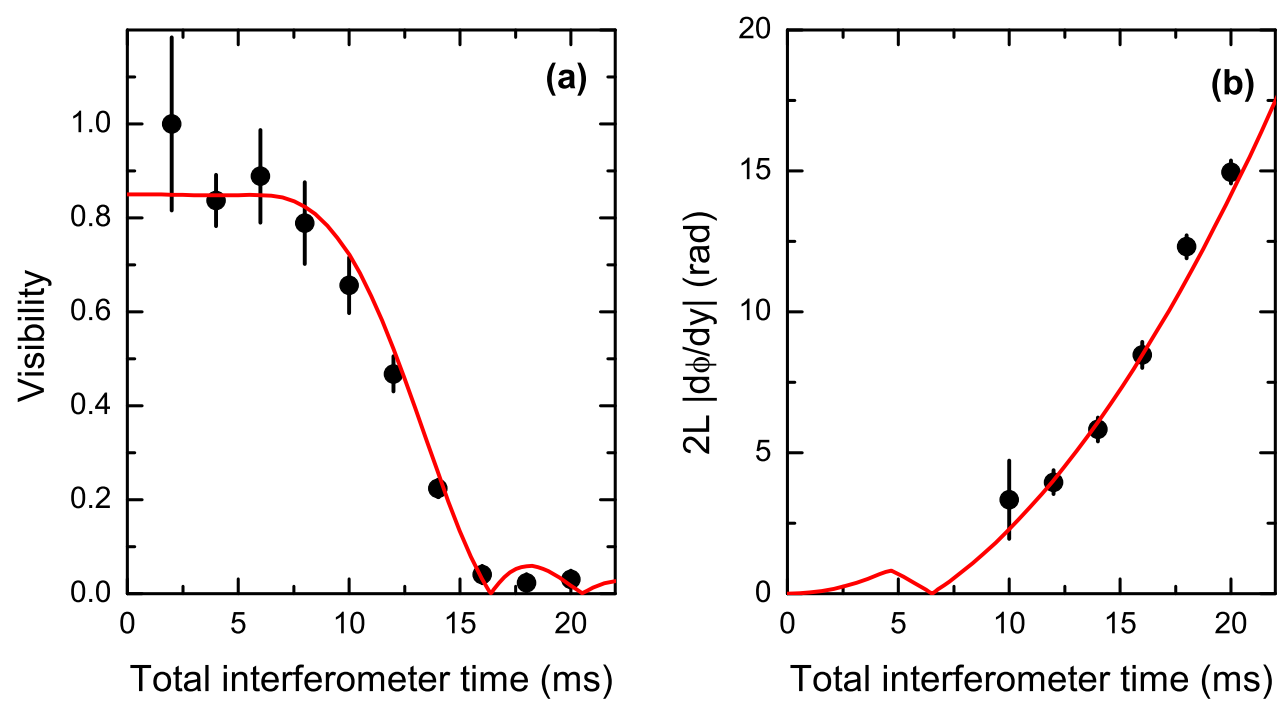

Figure 3.16: (a) Visibility of the single-sided interferometer as a function of total duration. Points show the measured values, while the curve shows the theoretical prediction. The prediction has been scaled by 0.85 to account for experimental imperfections from the splitting and reflection operations that are expected to be independent of duration. (b) Magnitude of the phase gradient for a single-sided interferometer of total duration $T$. Points show the experimental measurements, with error bars determined by the fitting procedure. The curve shows the results of the calculation described in the text, with the gradient scaled to the length of the packet $2 L$.

After taking a set of images in which the experimental conditions are kept the same but $\theta$ varied, we acquire the gradient $G=|d \phi / d \xi|$. For small gradients on the order of $1 / L$ or less, there is less spatial modulation, and therefore the fits become less reliable. Similarly, for large gradients, we are limited by the resolution of our imaging system and can no longer resolve the spatial modulation.

Fig. 3.16(b) shows the measured and calculated gradients as a function of the total interferometer time $T$. At short times, interaction effects contribute, causing the piecewise behavior of the calculated curve. The gradient from interactions varies across the cloud, here we use the gradient at the center of the cloud. The experimental data is seen to agree well with the calculation.

In general, to measure how well the interferometer works, we vary the applied phase $\theta$ and fit the resulting curve to a function $(1+V \cos \theta) / 2$. With phase gradients, we consider not only $\theta$ but 
also the phase $\phi$ from confinement and interaction effects. Using the one-dimensional density profile $n_{1 \mathrm{D}}$, we can calculate the fraction of atoms at rest after recombination as

$$
\begin{aligned}
\frac{N_{0}}{N} & =\frac{1}{2}\left[1+\int n_{1 \mathrm{D}}(\xi) \cos (\theta+\phi(\xi)) d \xi\right] \\
& =\frac{1}{2}\left[1+\int n_{1 \mathrm{D}}(\xi)(\cos \theta \cos \phi-\sin \theta \sin \phi) d \xi\right],
\end{aligned}
$$

where the second term will not give any contribution due to the density profile being symmetric in $\xi$. The visibility can then be found as

$$
V=\int n_{1 \mathrm{D}} \cos \phi(\xi) d \xi
$$

A measurement of the visibility of the interferometer, as well as the calculated visibility, can be seen in Fig. 3.16(a). Even at short times, we observe an imperfect visibility of around 0.85, and we scale the theoretical prediction by this factor. The calculated visibility is seen to agree well with the measured visibility. From the figure, one can see that the visibility drops to a value of 0.5 at $T \approx 12 \mathrm{~ms}$. This corresponds to a gradient of $4 \mathrm{rad}$ across the packets. At this time, the separation of the centers of the two packets is $120 \mu \mathrm{m}$, just barely larger than the full width of one of the clouds. The packets are therefore just separated, making a manipulation of the individual packets very difficult.

The possibility exists of operating at larger separations and therefore larger phase gradients. In this case, the fit parameter $\theta$ would be used as the output of the interferometer. However, this introduces a large amount of noise into the analysis, especially from the finite imaging resolution, as seen above. We therefore conclude that the performance of the single-sided interferometer is limited by confinement effects. 


\subsubsection{Double-sided interferometer}

We can cancel the phase gradient acquired in the one-sided interferometer by using a doublesided interferometer. In general, we can consider a double-sided, asymmetric interferometer with arm length $\tau_{1}$ in the first part of the interferometer followed by an arm length $\tau_{2}$ in the second part, as seen in Fig. 3.14(b). The total interferometer time is then given by $T=2 \tau_{1}+2 \tau_{2}$. In the symmetric case, $\tau_{1}=\tau_{2}=T / 4$.

The trajectories are given by Eq. (3.67) and (3.68) for $0<t<\tau_{1}$ and $\tau_{1}<t<2 \tau_{1}+\tau_{2}$, respectively. For $2 \tau_{1}+\tau_{2}<t<T$, the trajectory is

$$
\begin{aligned}
y_{+}^{\mathrm{III}}(t) & =\frac{v_{0}}{\omega}\left[-\sin \omega \tau_{1} \sin \omega\left(\tau_{1}+\tau_{2}\right)+\left[\cos \omega \tau_{1}-2\right] \cos \omega\left(\tau_{1}+\tau_{2}\right)+2\right] \sin \omega\left(t-2 \tau_{1}-\tau_{2}\right) \\
& +\frac{\nu_{0}}{\omega}\left[\sin \omega \tau_{1} \cos \omega\left(\tau_{1}+\tau_{2}\right)+\left[\cos \omega \tau_{1}-2\right] \sin \omega\left(\tau_{1}+\tau_{2}\right)\right] \cos \omega\left(t-2 \tau_{1}-\tau_{2}\right) .
\end{aligned}
$$

In the general asymmetric case, the phase difference can then be calculated as before to be

$$
\Delta \phi=4 k \xi\left\{\cos \left[2 \omega\left(\tau_{1}+\tau_{2}\right)\right]-2 \cos \left[\omega\left(\tau_{1}+2 \tau_{2}\right)\right]+2 \cos \omega \tau_{2}-1\right\}
$$

For short times $\omega T \ll 1$ and $\tau_{1}=\tau_{2}$, the phase difference can be estimated as

$$
\Delta \phi \approx k \xi(\omega T)^{4}
$$

which shows a suppression by a factor of $(\omega T)^{2}$ when compared to the single-sided case. The cancellation is not perfect due to the previously described velocity error $\delta v \propto(\omega T)^{2}$. The gradient due to interactions is similarly suppressed. For a symmetric interferometer with $\tau_{1}=\tau_{2}=\tau$ and $\tau>2.3 \mathrm{~ms}$, the gradient from interactions at the center of the packet can be calculated as $(\omega \tau)^{2}\left(m \omega^{2} L^{2} / \hbar v_{0}\right)$. At the longest times we achieve, $\tau \approx 20 \mathrm{~ms}$, the gradient is $0.025 \mathrm{rad} / L$, which is negligibly small.

The same result can be derived using the coherent state formalism described in the previous 

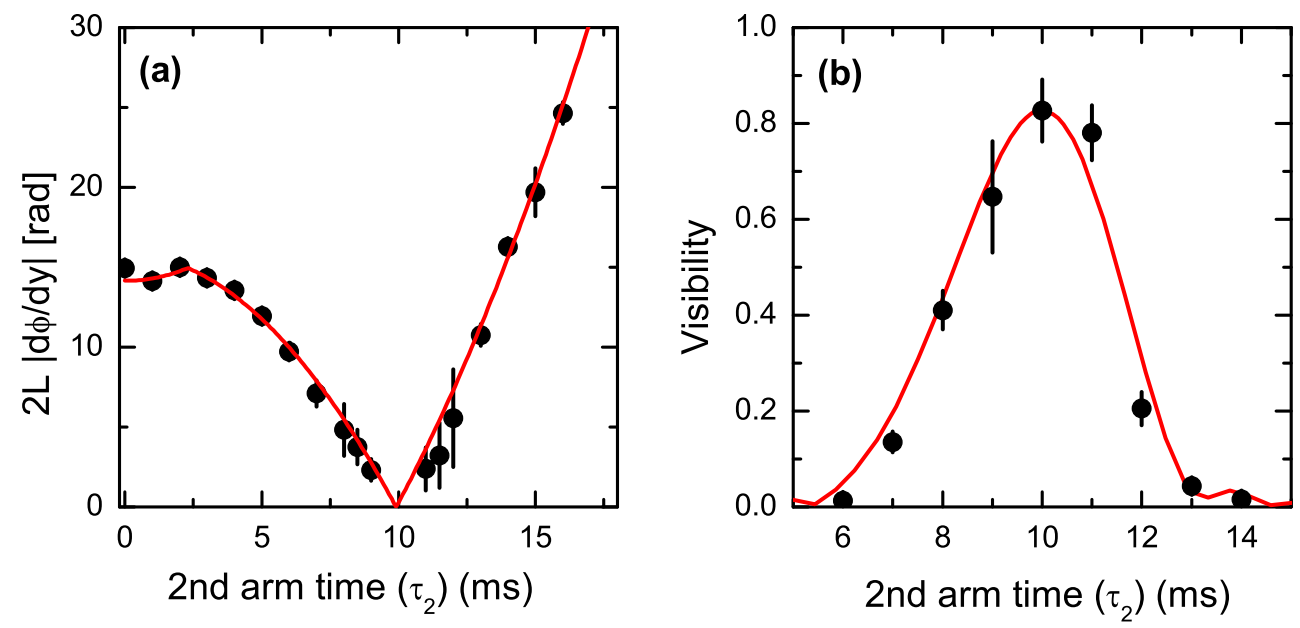

Figure 3.17: (a) Phase gradient observed in an asymmetric double-sided interferometer. The first half of the trajectory used leg time $\tau_{1}$ and the second half $\tau_{2}$. Here $\tau_{1}=10 \mathrm{~ms}$ and $\tau_{2}$ is varied. When $\tau_{2}=\tau_{1}$, the phase gradient is nearly eliminated. (b) Interferometer visibility as $\tau_{2}$ is varied. For both plots, data points show the experimental results and the curves show the model calculation. In (b), the calculation is scaled by 0.85 as in Fig. 3.16 .

section. Including the effect of an initial velocity for the double-sided symmetric interferometer with $T=4 \tau$ gives

$$
\Delta \phi=4 k\left[y_{1}(1-2 \cos \omega \tau+2 \cos 3 \omega \tau-\cos 4 \omega \tau)-\frac{\nu_{1}}{\omega}(2 \sin \omega \tau-2 \sin 3 \omega \tau+\sin 4 \omega \tau)\right],
$$

for an initial displacement $y_{1}$ and initial velocity $v_{1}$. Again, the coherent state calculation agrees with the semi-classical theory.

We can observe the cancellation effect by implementing an asymmetric interferometer with fixed $\tau_{1}=10 \mathrm{~ms}$ and varying $\tau_{2}$. In Fig. 3.17(a), the spatial density distribution is imaged and the gradient extracted, as before. It is seen that the gradient becomes so small as not to be measurable for the symmetric case $\tau_{1}=\tau_{2}$. Fig. 3.17(b) shows the corresponding peak in visibility for the symmetric case and the decay of interferometer performance at slight asymmetries. We note that this cancellation effect can be seen as analogous to the spin echo effect observed in magnetic resonance experiments [81]. 


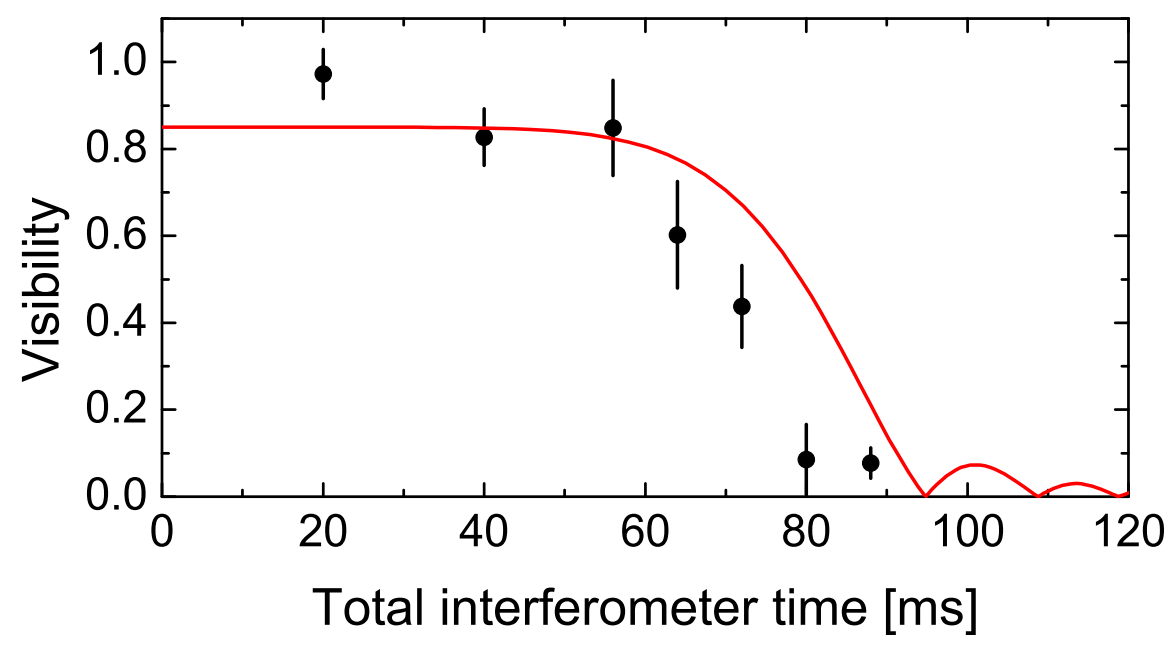

Figure 3.18: Visibility of the symmetric double-sided interferometer as a function of the total measurement time $T$. Data points show the experimental results and the curve shows the rigid packet prediction, scaled by 0.85 as in Fig. 3.16

In a symmetric double-sided interferometer, the cancellation of phase gradients therefore allows a significant improvement of measurement times in the interferometer. This is shown in Fig. 3.18, in which the calculated visibility is compared to the data shown previously in Fig. 3.12, We observe reasonably good agreement of the calculation with the experimental visibility. However, at longer times, the observed visibility consistently falls below the calculated values. At these longer times, neglecting the internal dynamics of the packets is obviously no longer justified.

An alternative to the approximation of a rigid packet is a model in which each atom undergoes free harmonic motion from its initial position in the packet. The packets therefore compress as they move up the confining potential. In the single-sided case, the two models give essentially the same result. In the double-sided case, the confinement phase is found to be

$$
\begin{aligned}
\Delta \phi=-k \xi[\cos 8 \omega \tau-2 \cos 7 \omega \tau+2 \cos 5 \omega \tau-2 \cos 3 \omega \tau+4 \omega \tau \sin 3 \omega \tau \\
+2 \cos \omega \tau-12 \omega \tau \sin \omega \tau-1]
\end{aligned}
$$


In the case of $\omega \tau \ll 1$, the gradient can be estimated as

$$
\frac{d \phi}{d \xi} \approx \frac{212}{3} k(\omega \tau)^{6}
$$

which is a higher order than the rigid packet model. However, the larger coefficient means that a coherence time of around $100 \mathrm{~ms}$ is found in this case, comparable to the rigid packet model.

In the rigid packet model, the repulsive interactions are assumed to be strong enough to keep the packet size fixed during the oscillation. At the other extreme, in the free oscillation model, interactions are neglected altogether. The reality probably lies somewhere in between these two cases. In addition, we do not take account of the three-dimensional nature of the system. In the transverse direction with tightest confinement, the oscillation period is $166 \mathrm{~ms}$, about twice the maximum interferometer time. Atomic interactions can couple motion in the transverse and longitudinal directions, causing changes in the length of the condensate on this timescale. Experimentally, we observe a change in packet size of around 15\%. This, of course, also affects the phase gradient across the cloud. A treatment of the internal dynamics would require a numerical solution of the three-dimensional Gross-Pitaevskii equation, and has not been performed here.

An indication that the simple theory developed here is not a sufficient description is that in the symmetric double-sided interferometer, the atom packets do not exhibit the spatial modulation with decreasing visibility as seen in Fig. 3.15. A possible explanation for this is that the reduction of visibility is caused by gradients of the phase in the transverse directions along with the longitudinal ones. We would not be able to resolve these in our experiment since we average over phase variations which are parallel to the probe beam in our absorption imaging setup.

Recently, a more complete analysis of the double-sided interferometer was performed by Stickney et al. [82]. In their analysis of our results, they attribute the loss in contrast to both the incomplete overlap of the packets and the phase gradient across the cloud. The combination of loss of contrast from these two sources could also be a possible explanation for the inability to 
see a spatial modulation in this case.

We have been able to rule out some further reasons for the decay. A misalignment of the standing-wave laser beam and the waveguide axis would cause the packets to be misaligned at recombination and not fully overlap, causing a reduction in interferometer visibility. We ruled out this possibility by imaging from all three directions and adjusting the alignment of the standingwave to make the atom packets overlap at recombination. Also, there is a possibility of overall phase noise, for example from vibrations in the standing-wave mirror. This would cause fluctuations in the interferometer output from shot to shot, which we do not observe. In fact, at an interferometer time of $T=100 \mathrm{~ms}$, the fluctuations in the fraction of atoms that remain at rest after recombination is only $1 \%$.

These results show that the double-sided interferometer offers a six-fold improvement in interferometer time over the single-sided interferometer, and a corresponding three-fold increase in arm separation. At these separations, the two packets are well separated and individually separated, offering the advantages described in Sec. 3.2.3. It should be noted that the double-sided interferometer scheme is not suitable for all measurements. For example, for a measurement of gravity, the double-sided interferometer would cancel the gravitational phase in the second half of the interferometer just like the confinement phase. However, a variety of other measurements are still possible, including the measurement of rotations with a loop geometry, or the measurement of effects that can be applied at a specified point in time. Measuring the ac or dc Stark effect belongs in the latter category.

\subsubsection{Free-oscillation interferometer}

Unfortunately, the same trick does not work to extend the interferometer time and arm separation even further. One could imagine realizing a '4-sided interferometer', in which the atoms are reflected four times before recombination. However, this results in a reduction of the phase gradient by only a factor of 4 , not by a power of $\omega T$, giving only a modest increase in the arm separation. At the same time, imperfections of the reflection operations become significant in 


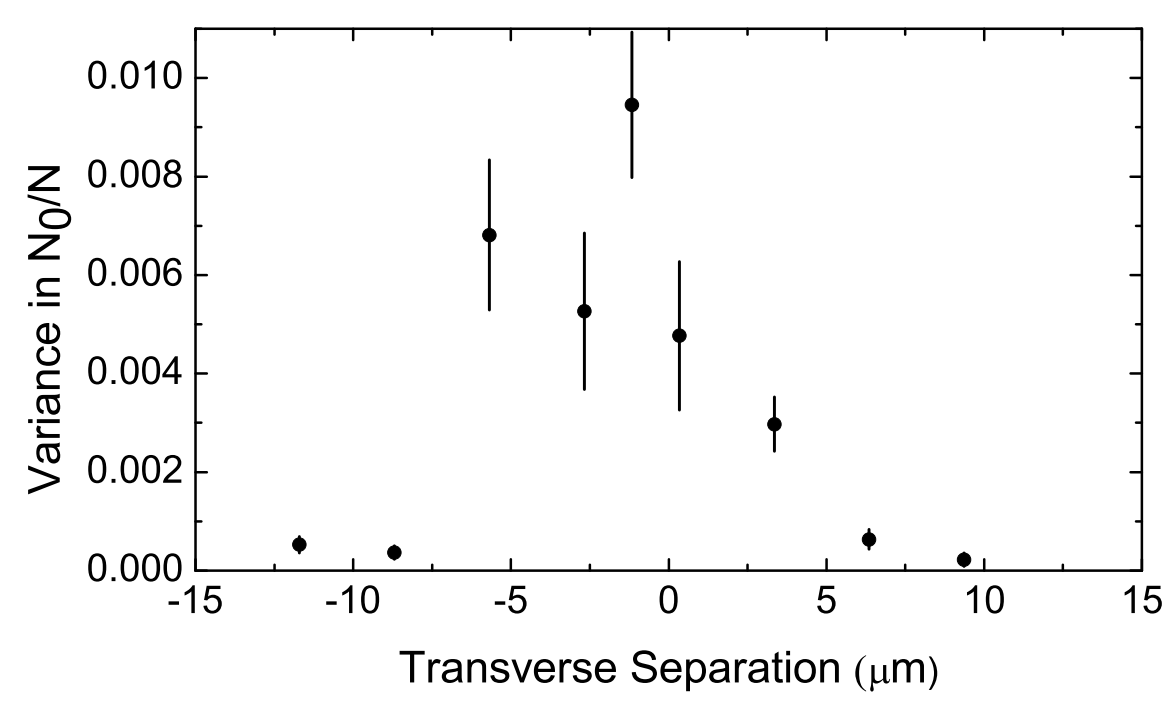

Figure 3.19: Interference for atoms freely oscillating in the waveguide. The measurement time is $0.9 \mathrm{~s}$, corresponding to a separation of $1.7 \mathrm{~mm}$. The phase of the interference is random, so the variance of the signal measured over several runs of the experiment is plotted against the transverse separation of the wavepackets at the time of the recombination pulse. The large increase in variance when the packets are overlapped is taken as a signal that interference is present. The error bars represent the error in the variance, estimated as $\Delta^{2} / N$.

this case. This geometry was therefore not studied in detail.

Thinking of the velocity error caused by the reflection operations caused us to pursue an interferometer without any reflection pulses. Instead, after splitting, the two packets complete a full oscillation in the confining potential before recombination. For an ideal harmonic potential, the trajectories of the packets are then precisely symmetric, and the phase gradients cancel exactly. However, the confining potential along the waveguide axis has an oscillation period of 0.9 s. At such long times, external noise sources can cause uncontrolled phase shifts of the packets. This means that we observe a random interferometer output.

Since we do not have a controlled interference signal, we instead use shot-to-shot fluctuations of the output as an indication of visibility. A similar technique was used in experiments by Segal et al. [83] and by Horikoshi and Nakagawa [84]. In both cases, fluctuations similar to ours were observed at long interferometer times. The arm separations obtained in this case were up 
to $0.5 \mathrm{~mm}$ and $180 \mu \mathrm{m}$, respectively. Horikoshi and Nakagawa were also able to operate this interferometer in a tighter confining trap with a shorter interferometer time and get a stable phase output. This indicates that low frequency noise such as that from vibrations of the table the experiment is mounted on are the cause of these fluctuations.

At such long oscillation times, the alignment of the standing-wave to the waveguide axis becomes critical in order to avoid the misalignment of the packets at the recombination time. We can also use the misalignment as a variable, as seen in Fig. 3.19. Here, we see a peak of the variance when the packets fully overlap, a clear indication of interference. We can quantify this by assuming an output of $(1+V \cos \theta) / 2$ of the interferometer with a random phase $\theta$. We can then relate the measured variance $\sigma^{2}$ to the visibility by $\sigma^{2}=V^{2} / 8$. This indicates an observed visibility of 0.3 at full overlap. The imperfect visibility could again be attributed to internal dynamics of the packets, which would cause a slight asymmetry in their trajectories. We note that we did not observe any interference after a half oscillation.

The maximum arm separation for the free-oscillation interferometer is $1.7 \mathrm{~mm}$. However, because of the random phase that is observed, there is no control over the output of the interferometer. We therefore cannot currently use this scheme to make practical measurements. It can be hoped that various stabilization methods will result in a controllable output, with which this interferometer would then offer an unprecedented interferometer time and arm separation.

In this section, we have seen that the performance of a guided matter-wave interferometer such as ours is largely limited by confinement effects at longer times. Since the confinement in the axial direction stems mainly from the effect of the current leads, as seen in Sec.2.2.3, an obvious solution would be to increase the length of the waveguide structure. An alternative method would be to introduce additional current elements which flatten out the waveguide potential in the axial direction, but add a small amount of confinement in the transverse directions. A calculation of the effect of such a configuration can be found in AppendixB. 


\subsubsection{Noise sources}

In the previous sections, some fundamental limitations to the interferometer performance were discussed. In addition to these fundamental limitations, there are also a range of possible sources of noise that contribute to a decreased interferometer performance. To compare the effect of these noise sources, we will always consider a symmetric, double-sided interferometer with $T=40 \mathrm{~ms}$ in the waveguide with confinement frequency $\omega_{y}=2 \pi \times 1.2 \mathrm{~Hz}$ along the guide axis.

\subsubsection{Trap field noise}

We can effectively eliminate the effects of fluctuating ambient fields on the interferometer by employing the time-orbiting potential technique described in Section 2.2 (for its limitations, see also Sec.3.3.4.3 below). However, if the fields of the TOP trap themselves fluctuate, this still introduces phase noise in the interferometer. Since these fields are generated electromagnetically, the main source of noise is the driving current. A characterization of the current noise is therefore important to understanding the stability of the interferometer.

Such a characterization has been carried out by Baranowski and Sackett [48], and here we follow the main points of their argument. We suppose that the energy of the atoms depends solely on the magnetic fields of the waveguide, i.e. $U=\mu B$, where $\mu$ is the magnetic moment of the atoms. Furthermore, any temporal variations in the fields are caused by current fluctuations, $B=\beta(y) I(t)$. We can then separate the current into a constant term and a fluctuating noise term $\eta(t)$ with $I(t)=\bar{I}[1+\eta(t)]$, where $\langle\eta(t)\rangle=0$ for the noise term.

Assuming the noise in the fields does not affect the trajectory of the atoms, we can develop $\beta$ into a Taylor series $\beta(y)=\sum \beta_{n} y^{n}$, where the even powers in the series do not contribute due to the symmetry of the trajectory. We then assume that the $n=1$ term will dominate and keep only this term, so that $\beta\left(y_{+}\right)-\beta\left(y_{-}\right) \approx 2 \beta_{1} y_{+}$. To simplify the notation, we will now redefine $\beta_{1}$ 
as $\beta_{1} \mu \bar{I}$. To leading order, the phase noise is then

$$
\delta \phi^{2}=\frac{4 \beta_{1}^{2}}{\hbar^{2}} H_{1} \int_{0}^{\infty} S(v) h_{1}(v) d v
$$

where $S(v)$ is the noise spectrum at a frequency $v$ (in $\mathrm{Hz}$ ), $H_{1}=v_{0}^{2} T^{3} / 48$ for a Bragg velocity $v_{0}=2 \hbar k / m$, and

$$
h_{1}(u)=\frac{3 T}{4 u^{4}}(\sin 2 u-2 \sin u)^{2},
$$

with $u=\pi v T / 2$ and the normalization $\int h_{1}(v) d v=1$.

The noise will typically have some noise floor and a sharp peak near the frequency of the line $v_{\text {line }}=60 \mathrm{~Hz}$. We can then write the noise spectrum as

$$
S(v)=S_{0}+\alpha \delta\left(v-v_{\text {line }}\right)
$$

which leads to phase noise

$$
\delta \phi^{2}=\frac{1}{3}\left(\frac{2 \beta_{1} v_{0} T}{4 \hbar}\right)^{2} T\left(\alpha h_{1}\left(v_{\text {line }}\right)+S_{0}\right) .
$$

We would expect $\beta_{1}=0$, since the atoms should be at a minimum of the potential. However, due to residual motion after loading the waveguide, they are not. At some initial position $y_{0}$, they experience a gradient $\beta_{1}=m \omega^{2} y_{0}$. When measuring the noise on the waveguide currents, we observe the noise floor at $-95 \mathrm{~dB} / \sqrt{\mathrm{Hz}}$ relative to the carrier and $60 \mathrm{~Hz}$ sidebands around the $11.9 \mathrm{kHz}$ peak at $-80 \mathrm{~dB}$ relative to the carrier. However, this is the square root of the power density. We therefore find $S_{0}=-190 \mathrm{~dB} / \mathrm{Hz}$ and $\alpha=-160 \mathrm{~dB}$. Using $v_{0}=2 \hbar k / m$, we then find the noise as

$$
\delta \phi=\sqrt{\frac{T}{3}} k y_{0} \omega^{2} T \sqrt{\alpha h_{1}\left(v_{\text {line }}\right)+S_{0}} .
$$

Inserting typical values of the initial position $y_{0} \approx 25 \mu \mathrm{m}$, we find $h_{1}\left(v_{\text {line }}\right)=1.7 \times 10^{-2} \mathrm{~T}$ and 


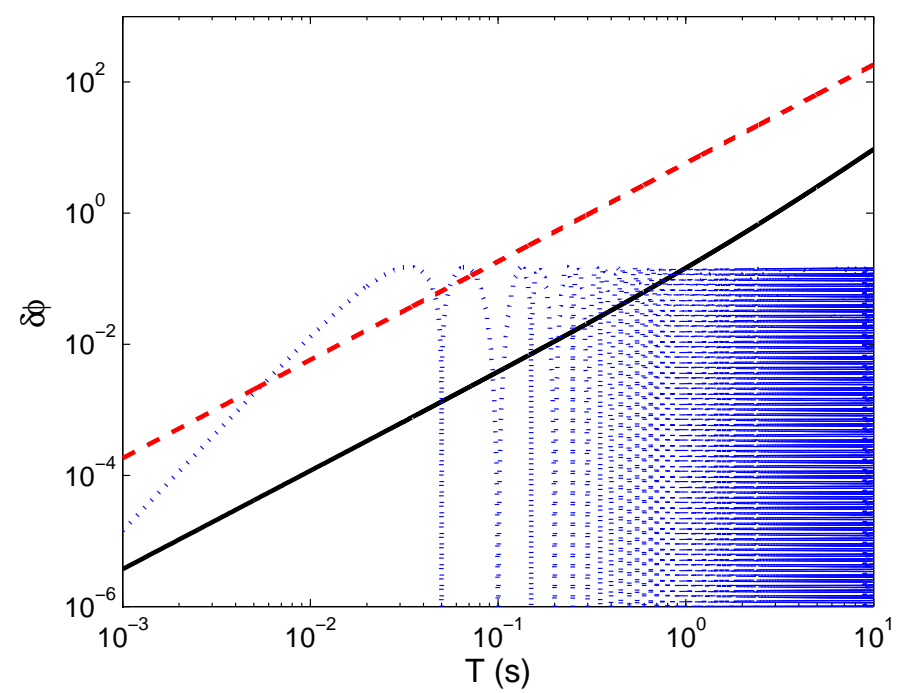

Figure 3.20: Comparison of the phase noise from various sources. The black solid line shows the effect of current noise using Eq. (3.96). The blue dotted line gives the effect of motion of the trap structure using Eq. (3.98), with $A=1 \mu \mathrm{m}$ and $v_{\mathrm{vib}}=40 \mathrm{~Hz}$. The red dashed line shows the effect of external field noise using Eq. (3.103), with $L=0.5 \mathrm{~m}$.

therefore a phase noise at $T=40 \mathrm{~ms}$ of

$$
\delta \phi=10^{-3} \mathrm{rad}
$$

The effect of current noise is therefore small enough to be negligible at these short times, and grows to unity for interferometer times of around $3 \mathrm{~s}$, as seen in Fig. 3.20 ,

\subsubsection{Motion of trap structure}

We can measure the motion of the trap structure by setting up an optical Fabry-Perot interferometer using a small mirror that is mounted on the trap structure. In this way, we measure an oscillation amplitude $A$ of a few $\mu \mathrm{m}$ with a frequency of around $50 \mathrm{~Hz}$. If the trap follows a trajectory $a(t)$, the atoms will see a varying potential $U(y, t)=m \omega^{2}(y-a)^{2} / 2 \approx U_{0}-m \omega^{2} a y$, which looks like a fluctuating gradient. We can therefore use the expression for phase noise from the previous section. At a frequency of $50 \mathrm{~Hz}, h_{1}$ turns out to be zero for $T=40 \mathrm{~ms}$, so let us assume 
a slightly smaller frequency of $40 \mathrm{~Hz}$. The phase noise is then given by

$$
\delta \phi=\frac{1}{\sqrt{3}} k A \omega^{2} T^{3 / 2} \sqrt{h_{1}\left(v_{\mathrm{vib}}\right)}
$$

This therefore results in a phase noise $\delta \phi \leq 0.5 \operatorname{rad}$ for $T=40 \mathrm{~ms}$.

\subsubsection{External field noise}

Noise from an external field $\mathbf{B}_{\mathbf{e}}$ will add to the trap potential and affect the atoms. Adding $\mathbf{B}_{\mathbf{e}}$ to the bias field given by Eq. 2.7) gives

$$
\left\langle\left|\mathbf{B}_{\mathrm{tot}}\right|\right\rangle \leq B_{0}+\frac{B_{e}^{2}}{2 B_{0}}
$$

A uniform field has no effect on the relative phase of the atoms, so consider a field with a gradient $B_{e}+B_{e}^{\prime} y$, which gives a potential gradient

$$
\frac{d U}{d y}=\frac{\mu B_{e} B_{e}^{\prime}}{B_{0}}
$$

With a gaussmeter, we can measure the spectrum of $B_{e}$, which we must then relate to the power spectrum $S(v)$. We will assume that the gradient of the field is given by $B_{e} / L$ for some length scale $L$, which should be more than a few $\mathrm{cm}$, since any possible sources are more than this distance away from the atoms. A measurement with a gaussmeter gives a noise background of $b_{2} \leq 1 \mathrm{mG} / \sqrt{\mathrm{Hz}}$, a peak at $60 \mathrm{~Hz}$ with amplitude $b_{1}=6 \mathrm{mG}$, and a static dc component $b_{0} \approx 1 \mathrm{G}$. The external field can then be expressed as

$$
B_{e}=b_{0}+b_{1} \cos \omega_{\text {line }} t+b_{2} n(t)
$$

where $n(t)$ is a white noise term with $\langle n(t) n(t+\tau)\rangle=\delta(\tau)$. The power spectrum for the squared 


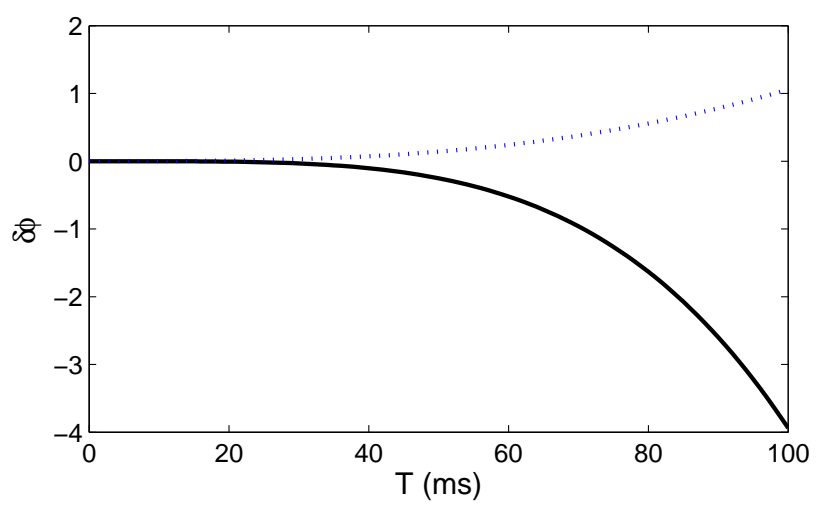

Figure 3.21: Comparison of the phase from residual motion of the atoms. The black solid line shows the effect of an initial displacement of $25 \mu \mathrm{m}$. The blue dotted line gives the effect of an initial velocity of $20 \mu \mathrm{m} / \mathrm{s}$.

field is then

$$
S_{e}(v)=b_{0}^{4} \delta(v)+4 b_{0}^{2} b_{1}^{2} \delta\left(v-v_{\text {line }}\right)+4 b_{0}^{2} b_{2}^{2}
$$

As before, the phase noise can then be calculated as

$$
\delta \phi^{2}=\frac{1}{3}\left(\frac{\mu v_{0} T b_{0}}{\hbar B_{0} L}\right)^{2} T\left[b_{1}^{2} h_{1}\left(v_{\text {line }}\right)+b_{2}^{2}\right]
$$

which gives a phase noise of $\delta \phi \approx 2.4 \mathrm{~cm} / L$ for the broadband term and $\delta \phi \approx 4 \mathrm{~mm} / L$ for the $60 \mathrm{~Hz}$ term. We therefore estimate $\delta \phi \leq 0.5 \mathrm{rad}$ for the contribution from external fields.

\subsubsection{Residual motion of atoms}

The effect of residual motion on the phase of the double-sided symmetric interferometer was calculated using the coherent state formalism, giving Eq. 3.89). Operating at an extremum of the oscillation in the guide should nominally give zero initial velocity, but we probably have fluctuations on the order of $0.1 \omega y_{1}=20 \mu \mathrm{m} / \mathrm{s}$. A typical value for the initial displacement at the extremum is $25 \mu \mathrm{m}$. The phase from the initial displacement is therefore $-0.1 \mathrm{rad}$ and the phase noise from a fluctuating initial velocity $0.07 \mathrm{rad}$ at $T=40 \mathrm{~ms}$, as can be seen in Fig. 3.21 , 
In the experiment, we do observe a constant phase shift which changes slowly over time, as mentioned in Chapter 4. Furthermore, we have observed a change in the phase offset of the interferometer curves for different interferometer times, which could arise from the phase shift from the initial displacement. In terms of phase fluctuations, we would be sensitive to fluctuations of the initial displacement, which varies by at least $10 \%$. The effect of residual motion will therefore limit interferometer performance for interferometer times longer than those which have already been achieved.

\subsubsection{Bragg beam noise}

As we saw earlier, changing the Bragg beam frequency changes the output signal of the interferometer as

$$
\frac{N_{0}}{N}=\frac{1}{2}[1+\cos (\phi+2 \alpha)]
$$

where $\phi$ is the differential phase and $\alpha=2 k D$ the standing-wave phase. The phase noise is then given by

$$
\delta \phi=2 \delta \alpha=4 D(\delta k)+4 k(\delta D)
$$

We monitor the Bragg beam with a Michelson interferometer, where the Bragg mirror is one of the mirrors of the interferometer, as seen in Fig. 3.1 The output of the interferometer is then given by

$$
I=\frac{I_{\max }}{2}(1+\sin 2 k L)
$$

where $L$ is the difference in length between the two arms of the interferometer. In our case $D=$ $22.5 \mathrm{~cm}$ and $L=35 \mathrm{~cm}$. Looking at the signal of the Michelson interferometer at $I_{\max } / 2$, the fluctuations are

$$
\delta I=I_{\max }(L \delta k+k \delta L)
$$


Since $L$ depends on the Bragg mirror as well as the other mirrors in the Michelson, we know that $\delta D \leq \delta L$, and therefore the phase noise is given by

$$
\delta \phi \leq 4 \frac{\delta I}{I_{\max }}
$$

We observe $\delta I / I_{\max } \leq 0.1$ on a timescale of around $40 \mathrm{~ms}$, giving a phase noise $\delta \phi \leq 0.4 \mathrm{rad}$.

\subsubsection{Table vibrations}

Fast vibrations of the optical table the experiment is performed on can also be thought of as a kind of Bragg beam noise. If the frequency of vibrations is larger than the trapping frequency, then the atoms themselves will not respond to this noise. However, the Bragg mirror is attached to the table and will therefore follow the vibrations. To the atoms, this then looks like a vibration of the Bragg mirror, with the same effect as in Sec. 3.3.4.5. The noise would not show up on the optical Michelson interferometer, though, since the other mirror and beamsplitter will be moving in the same way as the Bragg mirror.

A measurement of the table vibrations using geophones shows the largest contribution to the velocity noise at around $11 \mathrm{~Hz}$. The maximum velocity noise here is $2 \mu \mathrm{m} / \mathrm{s}$, giving a noise in $D$ of $\delta D=v / \omega=30 \mathrm{~nm}$. This corresponds to a phase noise of $\delta \phi=4 k \delta D=0.9 \mathrm{rad}$. This is the highest contribution to the phase noise.

Slow vibrations with a frequency less than the trapping frequency should not be a problem. The atoms experience slow vibrations as a constant acceleration, which will cancel due to the symmetry of the interferometer sequence.

For the current interferometer performance, it appears that table vibrations are the largest contribution to the phase noise in the experiment. At longer times, the residual motion of the atoms as well as possible noise from external fields will become important in limiting the performance of the interferometer. It would therefore be prudent to work at minimizing atomic 
oscillations even further, e.g. by working out the evaporation sequence with trap frequencies less than $60 \mathrm{~Hz}$ again, and actively canceling external magnetic fields. 
... incomparable blind streets of shuddering cloud

and lightning in the mind...

Allen Ginsberg

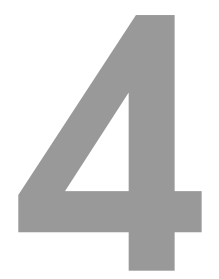

\section{Measuring the ac Stark effect}

Guided matter-wave interferometers have great potential for use in a variety of applications, from the precise measurement of inertial and gravitational effects to probes of chemical interactions $[24,85]$. While interference with matter waves in free space has yielded impressive results $[43,44]$, a considerable amount of space is needed for the atoms to fall under the influence of gravity or for an atomic beam to propagate. Using atoms confined in a guiding potential can solve this problem, and permits larger arm separations and enclosed areas using more flexible geometries such as circular rings $[45,46]$. So far, such guided-wave interferometers have been demonstrated in "proof-of-principle" experiments [53-55, 64, 65, 86, 87], but we show here that they can be of advantage for practical measurement as well. In this chapter, we report the use of a guided-wave interferometer to measure the dynamic polarizability of ${ }^{87} \mathrm{Rb}$ with an estimated accuracy of $7 \%$, a significant improvement over previous interferometric methods $[64,88,89]$. The accuracy is similar to that of the best non-interferometric technique [90], but in comparison our method requires a factor of $10^{2}$ less laser power and can be used at any optical frequency, including on resonance. Indeed, measurement on resonance reveals the familiar dispersionshaped dependence, but with a broadened linewidth that we attribute to collective scattering effects $[91,92]$.

When atoms interact with the electric field of a beam of light, the energy levels of the atoms

shift due to the ac Stark effect by an amount $U=-\frac{1}{2} \alpha\left\langle E^{2}\right\rangle$. This effect is relevant to precision measurements, where the magnitude of such shifts must be well known [93, 94], and measure- 
ments of the polarizability $\alpha$ are important for verifying ab initio calculations of dipole matrix elements [95]. Gradients of the Stark potential lead to optical dipole forces, which are used to great advantage for cooling and trapping atoms [96], atom optics [23], as well as quantum information [97].

\subsection{Theory}

When an atom experiences an electric field $\mathscr{E}$, the applied field distorts the charge distribution in the atom and therefore induces an electric dipole moment $\boldsymbol{\mu}=\alpha \mathscr{E}$. The interaction potential for a dipole with an electric field is given by

$$
U=-\frac{1}{2}\langle\boldsymbol{\mu} \cdot \mathscr{E}\rangle
$$

where the factor of $1 / 2$ comes from the fact that the dipole moment is induced [98]. Combining the expression for the energy shift with the expression for the intensity of a beam of light,

$$
I=c \epsilon_{0}\left\langle\mathscr{E}^{2}\right\rangle
$$

gives the potential

$$
U=-\frac{1}{2 c \epsilon_{0}} \alpha I
$$

A simple model To get an estimate for the polarizability, we can use a simple classical model for atom interactions with light [98]. An electron with mass $m_{e}$ and elementary charge $e$ can be thought of as a classical oscillator bound to the core with an oscillation frequency $\omega_{0}$, which is equal to the frequency of the optical transition. The oscillator is driven by the force from an electric field $\mathscr{E} \propto \exp (-i \omega t)$. The restoring force for the oscillator in this case is just

$$
\mathbf{F}=-m_{e} \omega_{0}^{2} \mathbf{x}
$$


Adding a damping term $\gamma$ then gives us the equation of motion for a driven, damped classical oscillator

$$
m_{e}\left[\ddot{\mathbf{x}}+\gamma \dot{\mathbf{x}}+\omega_{0}^{2} \mathbf{x}\right]=-e \mathscr{E},
$$

which gives a dipole moment of

$$
\boldsymbol{\mu}=\frac{e^{2}}{m_{e}}\left(\omega_{0}^{2}-\omega^{2}-i \omega \gamma\right)^{-1} \mathscr{E}
$$

The radiative damping rate of a classical oscillator [98] is found to be $\gamma=e^{2} \omega_{0}^{2} / 6 \pi \epsilon_{0} m_{e} c^{3}$, which can be used to replace $e^{2} / m_{e}$ in Eq. (4.6) and give an expression for the dipole moment of

$$
\boldsymbol{\mu}=\frac{6 \pi \epsilon_{0} c^{3}}{\omega_{0}^{2}} \frac{\gamma}{\omega_{0}^{2}-\omega^{2}-i \omega \gamma} \mathscr{E}
$$

This is an accurate description of the dipole moment for a suitable quantum mechanical definition of $\gamma$.

Semi-classical theory A more rigorous treatment of the atomic interaction can be performed using time-dependent perturbation theory. We will try to find solutions to the Schrödinger equation

$$
i \hbar \frac{d}{d t}|\Psi\rangle=H|\Psi\rangle
$$

where the Hamiltonian is given as $H=H_{a}+V(t)$, with the unperturbed atomic Hamiltonian $H_{a}$ and a time-dependent perturbation $V=-\boldsymbol{\mu} \cdot \mathscr{E} \cos \omega t$. With the definition of the Rabi frequency

$$
\mathbf{\Omega}=\langle i|\boldsymbol{\mu}| f\rangle \mathscr{E} / \hbar
$$

for an initial state $|i\rangle$ and a final state $|f\rangle$, we can separate the perturbation into two parts

$$
V=V_{+} e^{-i \omega t}+V_{-} e^{i \omega t}
$$


with $V_{+}=V_{-}=\hbar \Omega / 2$.

The problem of linear differential equations with periodic coefficients can often be solved using Floquet theory $[99,100]$. This is a similar approach as the Bloch theorem in condensed matter physics. We start with the Floquet ansatz

$$
|\Psi(t)\rangle=e^{-i E_{m} t / \hbar}\left|\psi_{m}(\tau)\right\rangle
$$

where $E_{m}$ is the quasi-energy, and $\left|\psi_{m}(\tau)\right\rangle$ is periodic in $\tau=\omega t$. The subscript $m$ denotes the $m$-th Floquet mode. Due to the periodicity, the state $\left|\psi_{m}(\tau)\right\rangle$ can be expanded as

$$
\left|\psi_{m}(\tau)\right\rangle=\sum_{n} e^{-i n \omega t}\left|\psi_{m n}(\tau)\right\rangle
$$

where $n \in \mathbb{Q}$.

Inserting this into the Schrödinger equation (4.8), we find

$$
\begin{aligned}
E_{m} \sum_{n} e^{-i n \omega t}\left|\psi_{m n}\right\rangle+ & \sum_{n} n \hbar \omega e^{-i n \omega t}\left|\psi_{m n}\right\rangle= \\
& H_{a} \sum_{n} e^{-i n \omega t}\left|\psi_{m n}\right\rangle+V_{+} \sum_{n} e^{-i(n-1) \omega t}\left|\psi_{m n}\right\rangle+V_{-} \sum_{n} e^{-i(n+1) \omega t}\left|\psi_{m n}\right\rangle .
\end{aligned}
$$

Since the solution should be valid for all times, we compare the terms oscillating at the same frequency and get the coupled equation [101]

$$
\left(E_{m}+n \hbar \omega-H_{a}\right)\left|\psi_{m n}\right\rangle=V_{+}\left|\psi_{m(n-1)}\right\rangle+V_{-}\left|\psi_{m(n+1)}\right\rangle
$$

We can now expand the quasi-energy and the state vector in a series in terms of the electric field $\mathscr{E}$ as

$$
\begin{gathered}
E_{m}=E^{(0)}+E^{(1)} \mathscr{E}+E^{(2)} \mathscr{E}^{2}+\ldots \\
\left|\psi_{m n}\right\rangle=\left|\psi_{n}^{(0)}\right\rangle+\mathscr{E}\left|\psi_{n}^{(1)}\right\rangle+\mathscr{E}^{2}\left|\psi_{n}^{(2)}\right\rangle+\ldots
\end{gathered}
$$


In the unperturbed case, we have $H_{a}\left|\psi_{0}^{(0)}\right\rangle=E^{(0)}\left|\psi_{0}^{(0)}\right\rangle$, and therefore $E^{(0)} \equiv E$ and $\psi_{n}^{(0)}=0$ for all $n \neq 0$.

We can now look at the first order correction. We find

$$
E^{(1)}\left|\psi_{n}^{(0)}\right\rangle+\left(E \pm n \hbar \omega-H_{a}\right)\left|\psi_{n}^{(1)}\right\rangle=V_{+}\left|\psi_{n-1}^{(0)}\right\rangle+V_{-}\left|\psi_{n+1}^{(0)}\right\rangle
$$

For $n=0$, we see after multiplying with $\left\langle\psi_{0}^{(0)}\right|$ that the first correction to the energy $E^{(1)}$ is zero. For $n= \pm 1$, on the other hand, the first order wavefunction is given by

$$
\left|\psi_{ \pm 1}^{(1)}\right\rangle=G(E \pm \hbar \omega) V_{ \pm}\left|\psi_{0}^{(0)}\right\rangle
$$

with the Green's function

$$
G(E \pm \hbar \omega)=\left(E \pm \hbar \omega-H_{a}\right)^{-1}
$$

To second order, for $n=0$, we find

$$
E^{(2)}\left|\psi_{0}^{(0)}\right\rangle+\left(E-H_{a}\right)\left|\psi_{0}^{(2)}\right\rangle=V_{+}\left|\psi_{-1}^{(1)}\right\rangle+V_{-}\left|\psi_{+1}^{(1)}\right\rangle
$$

from which we get an expression for the second order correction to the energy as

$$
E^{(2)}=\left\langle\psi_{0}^{(0)}\left|V_{+}\right| \psi_{-1}^{(1)}\right\rangle+\left\langle\psi_{0}^{(0)}\left|V_{-}\right| \psi_{+1}^{(1)}\right\rangle
$$

We can now substitute Eq. (4.19) into this expression and find

$$
E^{(2)}=\left\langle\psi_{0}^{(0)}\left|V_{+} G(E-\hbar \omega) V_{-}\right| \psi_{0}^{(0)}\right\rangle+\left\langle\psi_{0}^{(0)}\left|V_{-} G(E+\hbar \omega) V_{+}\right| \psi_{0}^{(0)}\right\rangle
$$

where the first term corresponds to an atom emitting a photon, then absorbing one, and the second term corresponding to an absorption of a photon followed by an emission. By making 
use of completeness, we can now express the Green's function as

$$
\frac{1}{E \pm \hbar \omega-H_{a}}=\sum_{f} \frac{|f\rangle\langle f|}{E \pm \hbar \omega-E_{f}}
$$

Taking advantage of $V_{+}=V_{-}$, the energy shift can now be written as

$$
E^{(2)}=\sum_{f} \frac{\left|\left\langle\psi_{0}^{(0)}\left|V_{+}\right| f\right\rangle\right|^{2}}{\hbar}\left(\frac{1}{\omega_{f}-\omega}+\frac{1}{\omega_{f}+\omega}\right)
$$

where we have defined $\hbar \omega_{f} \equiv E-E_{f}$. Using $V_{+}=\hbar \Omega / 2$, this can then be written as

$$
E^{(2)}=\sum_{f} \frac{\hbar \Omega^{2}}{4}\left(\frac{1}{\omega_{f}-\omega}+\frac{1}{\omega_{f}+\omega}\right)=\sum_{f} \frac{\hbar \Omega^{2}}{2} \frac{\omega_{f}}{\omega_{f}^{2}-\omega^{2}}
$$

The second order energy shift is simply the energy shift from a beam of light given in Eq. 4.3). From this equation, we can then extract the polarizability $\alpha$. Using the definitions of the Rabi frequency (4.9) and the intensity (4.2), we find

$$
U=-\sum_{f} \frac{I}{\hbar c \epsilon_{0}}|\langle i|\mu| f\rangle|^{2} \frac{\omega_{f}}{\omega_{f}^{2}-\omega^{2}},
$$

where we have ignored spontaneous emission, which could be included by adding an imaginary term to the denominator as in Eq. 4.7.

Our experiment uses ${ }^{87} \mathrm{Rb}$, which has two principal transitions from the $5 S_{1 / 2}$ ground state, to the $5 P_{1 / 2}$ state at $795 \mathrm{~nm}$, and to the $5 P_{3 / 2}$ state at $780 \mathrm{~nm}$. Each of these states has several hyperfine levels, which we must consider. From Eq. (4.26), the polarizability of an atomic state $|i\rangle$ is found as [36]

$$
\alpha_{i}\left(\omega_{\ell}\right)=\frac{2}{\hbar} \sum_{f \neq i} \frac{\omega_{f}}{\omega_{f}^{2}-\omega_{\ell}^{2}}\left|\mu_{i f}\right|^{2},
$$

where $\omega_{\ell}$ is the frequency of the applied laser beam, $\omega_{f}$ is the transition frequency to an excited state $|f\rangle$, and $\mu_{i f}$ is the transition dipole matrix element $\langle i|\mu| f\rangle$. This matrix element can be 


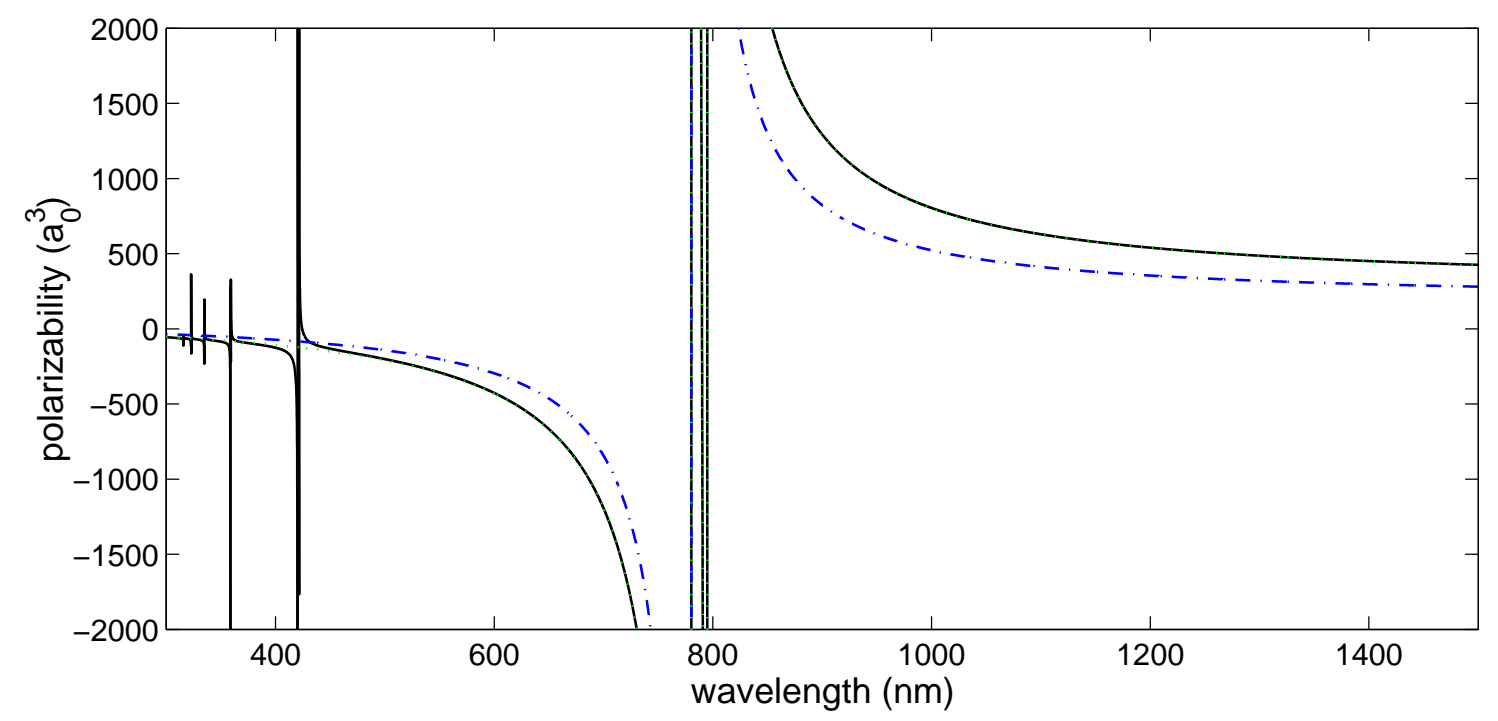

Figure 4.1: Polarizability of the $5 S_{1 / 2}$ ground state of ${ }^{87} \mathrm{Rb}$. The black curve shows the contributions from the 12 strongest transitions, as given by [102]. The blue dot-dashed line is the contribution from the $5 P_{3 / 2}$ state. After including the $5 P_{1 / 2}$ state (green dotted line), the other states' contributions are very small.

expressed in terms of the decay rate $\Gamma_{f i}$ of the excited state using

$$
\Gamma_{f i}=\frac{\omega_{f}^{3}}{3 \pi \epsilon_{0} \hbar c^{3}}\left|\mu_{i f}\right|^{2}
$$

We use this expression to normalize the dipole moments to the $5 P_{3 / 2} \quad\left(F=3, m_{F}=3\right) \rightarrow 5 S_{1 / 2}$ $\left(F=2, m_{F}=2\right)$ cycling transition rate, where $\Gamma_{33 \rightarrow 22}=\Gamma=2 \pi \times 6.065 \mathrm{MHz}$ is the total decay rate for the $5 P_{3 / 2}$ states [103]. Then

$$
\alpha_{i}\left(\omega_{\ell}\right)=4 \pi \epsilon_{0} \frac{3 \Gamma c^{3}}{2 \omega_{33}^{3}} \sum_{f \neq i} \frac{\omega_{f}}{\omega_{f}^{2}-\omega_{\ell}^{2}}\left|\frac{\mu_{i f}}{\mu_{33}}\right|^{2} .
$$

The ratios of dipole moments can be computed exactly from standard angular momentum algebra, and are tabulated in the literature $[13,104]$. The relative intensities of the transitions used in our calculation are given in Table 4.1 . 
(a) Transitions to excited states

\begin{tabular}{lcc}
\hline \hline Excited state & wavelength $(\mathrm{nm})$ & $\left|\mu_{i f} / \mu_{33}\right|^{2}$ \\
\hline $5^{2} P_{1 / 2}$ & 794.9783 & $3 / 8$ \\
$5^{2} P_{3 / 2}$ & 780.2405 & $3 / 4$ \\
$6^{2} P_{1 / 2}$ & 421.6706 & $1 / 240$ \\
$6^{2} P_{3 / 2}$ & 420.2972 & $1 / 120$ \\
$7^{2} P_{1 / 2}$ & 359.2597 & $1 / 3000$ \\
$7^{2} P_{3 / 2}$ & 358.8074 & $1 / 1200$ \\
\hline \hline
\end{tabular}

(b) Transitions to different hyperfine levels

\begin{tabular}{lccc}
\hline \hline Excited state & Hyperfine level & $\omega_{f} / 2 \pi(\mathrm{THz})$ & $\left|\mu_{i f} / \mu_{33}\right|^{2}$ \\
\hline $5^{2} P_{1 / 2}$ & 2 & 377.105206740 & $1 / 3$ \\
$5^{2} P_{3 / 2}$ & 2 & 384.227848551 & $1 / 3$ \\
$5^{2} P_{3 / 2}$ & 3 & 384.228115203 & $1 / 3$ \\
\hline \hline
\end{tabular}

Table 4.1: Relative intensities of transitions used in calculating the ac polarizability of the $5^{2} S_{1 / 2}$ ground state of ${ }^{87} \mathrm{Rb}$. (a) Transitions from the ground state to higher lying electronic states, averaged over the hyperfine structure. The relative intensities are given by the square of the ratio of the dipole matrix element of the transitions to that of the $5 P_{3 / 2}\left(F=3, m_{F}=3\right) \rightarrow 5 S_{1 / 2}(F=$ $\left.2, m_{F}=2\right)$ cycling transition. The relative intensities are from Ref. [102]. (b) Transitions from the ground state to different hyperfine levels of the first excited electronic state. The relative intensities in this case are from Ref. [13].

\subsection{Experiment}

Our apparatus has been described in detail in previous chapters. Briefly, we have a Bose-Einstein condensate of $3 \times 10^{4}{ }^{87} \mathrm{Rb}$ atoms in a harmonic waveguide generated by a time-orbiting potential (TOP) with transverse confinement frequencies of $3.3 \mathrm{~Hz}$ and $6 \mathrm{~Hz}$ and axial confinement of 1.1 Hz. An off-resonant standing-wave laser beam is used to evenly split the condensate with almost perfect efficiency into two packets which move in opposite directions along the waveguide axis with velocity $v_{0}=11.7 \mathrm{~mm} / \mathrm{s}$. We use the symmetric, double-sided interferometer, of which the trajectories of the packets are shown in Fig. 3.6. For this type of interferometer, the two packets of atoms traverse nearly the same paths, therefore experiencing the same phase shifts from the guide potential, as described in Sec. 3.3.3. After recombination, the ratio of the number of 


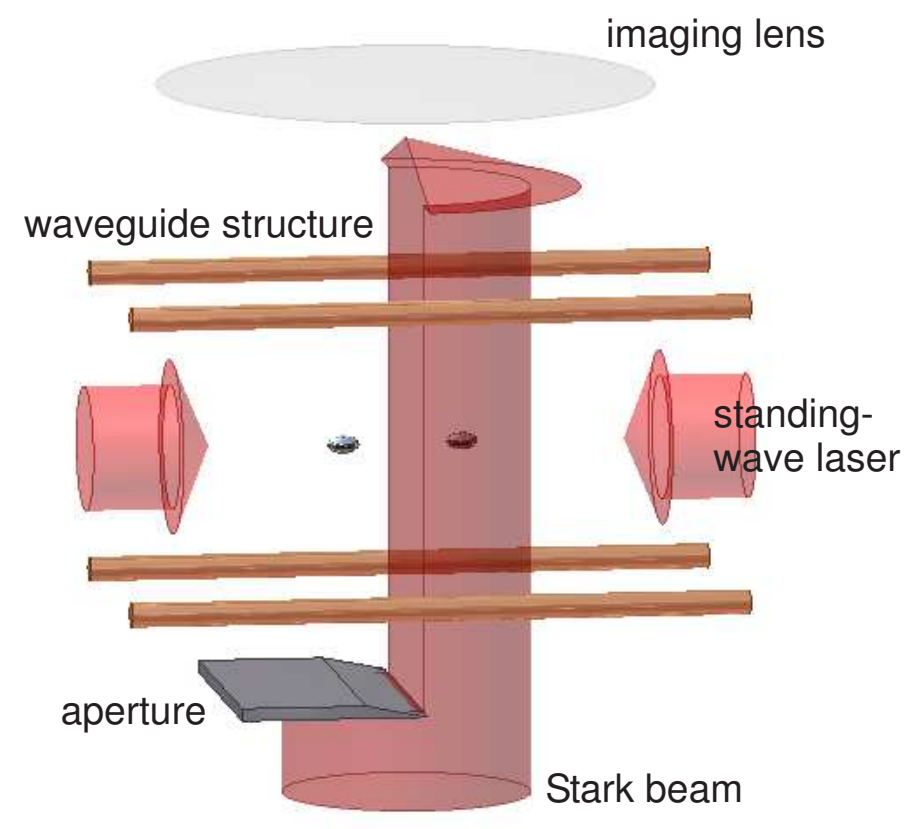

Figure 4.2: Schematic of the experimental setup. The waveguide axis is in the horizontal direction. The Stark beam is apertured such that it only interacts with one packet of atoms at the maximum separation in the interferometer. This beam is imaged with the same camera that we use to observe the atoms.

atoms at rest $N_{0}$ to the total number of atoms $N$ depends on the differential phase $\phi$,

$$
\frac{N_{0}}{N}=\frac{1}{2}(1+\cos \phi)
$$

For the experiments discussed here, the interferometer time is always $T=40 \mathrm{~ms}$, for which we have nearly perfect visibility, as seen in Fig. 3.12, The maximum center-to-center separation of the two packets for this distance is $240 \mu \mathrm{m}$, which is significantly larger than the cloud halfwidth of $55 \mu \mathrm{m}$ and allows for independent access to the two packets. This allows us to apply a laser beam (the "Stark beam") to one atom packet but not the other, as seen in Fig.4.2.

In the experiment, the Stark beam is linearly polarized along the waveguide axis and is spatially filtered with a pinhole. An AOM is used to pulse on the Stark beam for a short time before and after the first reflect pulse, and a mechanical shutter is used to prevent any leakage light 

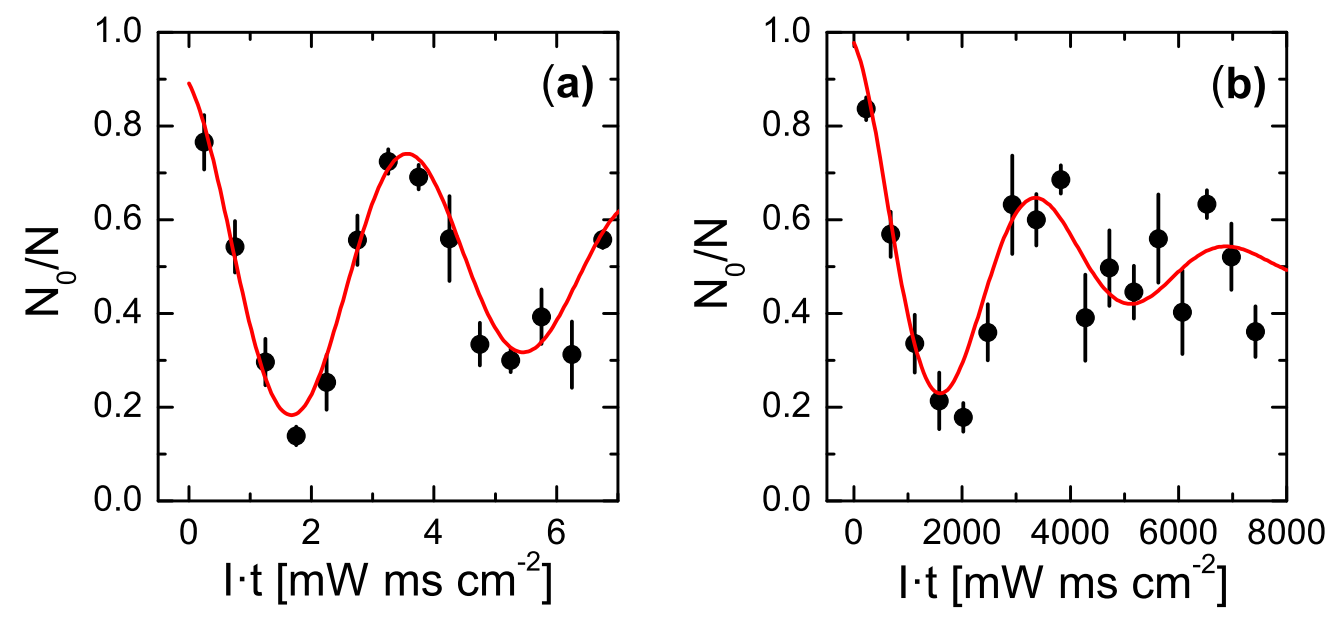

Figure 4.3: Measurement of the phase shift from a laser beam at (a) $780.232 \mathrm{~nm}$ and (b) $808.37 \mathrm{~nm}$. In both graphs, the solid line indicates a fit to the data. Since there is an increasing phase gradient across the atomic cloud with increasing phase, the visibility decays at higher intensities and longer times. In the graph, we have binned together groups of data with a similar product of measured intensity and pulse time. The bin size is $0.5 \mathrm{~mW} \mathrm{~ms} \mathrm{~cm} \mathrm{~mW}^{-2}$ in (a), $450 \mathrm{~mW}$ $\mathrm{ms} \mathrm{cm}^{-2}$ in (b), and the error bars indicate the standard deviation of the mean within the bin.

from the AOM from affecting the atoms. In addition, the power in the Stark beam is measured during the applied pulse with a photodiode and the intensity corrected accordingly to account for variations over time, which are generally less than $10 \%$. After completing the interferometer sequence and recombining the two clouds, the ratio $N_{0} / N$ is measured for different intensities and durations of the Stark beam.

\subsubsection{Measurement of the ac polarizability off resonance}

We performed this experiment at two different frequencies. For the first measurement, as seen in Fig. 4.3 (a), laser light locked to the $5 S_{1 / 2}, F=1$ to $5 P_{3 / 2}, F=2$ "repump" transition at $780.232 \mathrm{~nm}$ was used as the Stark beam. In two variations, a $475 \mu$ s pulse was applied and the Stark beam intensity $I$ varied from 1 to $15 \mathrm{~mW} / \mathrm{cm}^{2}$, or a beam with intensity $10.5 \mathrm{~mW} / \mathrm{cm}^{2}$ was applied for 
times $t$ ranging from 0 to $675 \mu \mathrm{s}$. We fit the data to a function

$$
f(I t)=\frac{1}{2}+e^{-\beta I t} \cos \left(2 \pi \frac{I t-x_{0}}{P}\right)
$$

where $P$ is the period, $x_{0}$ is an overall phase offset, and $\beta$ is a decay constant reflecting the fact that intensity gradients in the beam induce spatial variations in the phase which eventually wash out the interference. Here the unapertured beam waist was $1 \mathrm{~mm}$. We can extract the polarizabilities from the fit using $|\alpha|=4 \pi \epsilon_{0} c \hbar / P$. For the data at $780.232 \mathrm{~nm}$, the fit gives $|\alpha|=4 \pi \epsilon_{0} \times(8.37 \pm 0.24) \times 10^{-25} \mathrm{~m}^{3}$, where the error is the statistical error from the fit. In order to get a theoretical value, we consider only transitions from the $5 S_{1 / 2}$ ground state to the $5 P_{3 / 2}, F=2,3$ excited states. The contributions from the Zeeman effect induced by the $20 \mathrm{G}$ trap bias field and from $5 P_{1 / 2}$ states are less than $0.1 \%$. Our calculations give a theoretical value of $|\alpha|=4 \pi \epsilon_{0} \times 8.67 \times 10^{-25} \mathrm{~m}^{3}$, a deviation of $+3.5 \%$ relative to the experiment.

For the second measurement, seen in Fig.4.3(b), a free running laser diode at $808.37 \mathrm{~nm}$ was used. As in the previous case, a $1.276 \mathrm{~ms}$ pulse with intensities ranging from 0 to $6.6 \mathrm{~W} / \mathrm{cm}^{2}$ was applied to the atoms, or a beam with intensity $4.6 \mathrm{~W} / \mathrm{cm}^{2}$ was applied for various times from 0 to $1.376 \mathrm{~ms}$. For this data, the fit gives $|\alpha|=4 \pi \epsilon_{0} \times(9.48 \pm 0.25) \times 10^{-28} \mathrm{~m}^{3}$. Here, the Stark beam was focused down to a waist of $0.2 \mathrm{~mm}$, leading to higher intensities but also higher intensity gradients across the cloud. This explains the faster decay in the data at $808 \mathrm{~nm}$ than at $780 \mathrm{~nm}$. A theoretical value was calculated using contributions from transitions to the $6 P$ as well as the $5 P$ states. Here, contributions from the hyperfine structure of the excited states and from higher $P$ states are less than $0.01 \%$, and are neglected. The calculated value for the polarizability is then $|\alpha|=\left(4 \pi \epsilon_{0}\right) \times 9.14 \times 10^{-28} \mathrm{~m}^{3}$, differing from the measured value by $-3.7 \%$. In both cases, we attribute the deviation between experiment and theory to inaccuracy of the intensity calibration. Somewhat curiously, the errors on the polarizability extracted from the fit are of similar size, even though there is significantly more scatter in the data at $808 \mathrm{~nm}$ than at $780 \mathrm{~nm}$. We confirmed that this was indeed the case by fitting to subsets of data, for which the error was increased in 
both cases.

As in most measurements of polarizability, the calibration of the applied field is the primary source of uncertainty. In our technique, however, the atoms are well-localized within the Stark beam and a single camera is used to observe both the beam and the atoms. This allows a relatively precise determination of the actual intensity applied. We calibrate the camera by comparing the sum of the pixel values in an image of the unapertured Stark beam to the total power as measured by an optical power meter. Alternatively, the picture of the unapertured beam can be fit to a 2-dimensional Gaussian distribution to find its center and width, and the intensity at the atoms estimated as

$$
I(x, y)=\frac{2 P}{\pi w_{x} w_{y}} \exp \left(-\frac{\left(x-x_{0}\right)^{2}}{w_{x}^{2}}-\frac{\left(y-y_{0}\right)^{2}}{w_{y}^{2}}\right)
$$

for measured total power $P$. This method averages over high-frequency spatial noise in the beam, some of which is introduced by the imaging optics and is therefore not present on the atoms. The intensities obtained with the two methods differ with a standard deviation of $4 \%$, and we use their average for the calibration. This uncertainty might be reduced by use of an imaging system with fewer optical elements.

The other main source of error is the calibration of the Ophir PD200 power meter, which has a specified accuracy of $5 \%$ at $780 \mathrm{~nm}$. This is consistent with the difference observed when we compared the PD200 to a Coherent Lasermate meter. This error could be reduced significantly by using an optical reference standard [105]. Altogether, we estimate the total uncertainty for the measurement to be $7 \%$, which can be compared to the $8 \%$ error cited by Kadar-Kallen et al. [90]. Turning our result around, the fact that the dipole moment for the ${ }^{87} \mathrm{Rb}$ transition is known accurately means that our technique could instead be used as precise measurement of the Stark beam intensity at the atoms, with an uncertainty of about $2.5 \%$. 

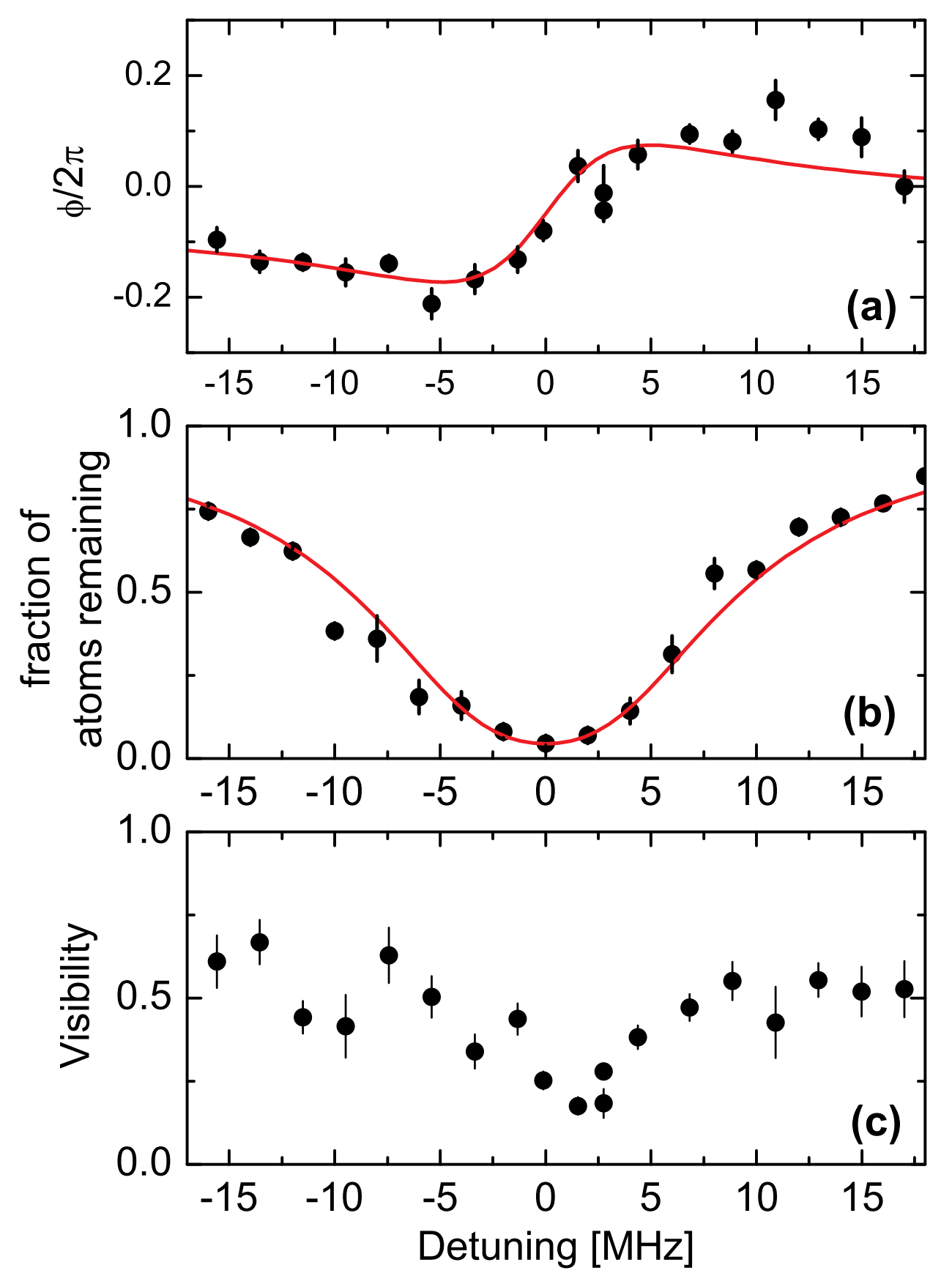

Figure 4.4: (a) Measurement of the phase shift from a laser beam near the atomic resonance. (b) Loss of atoms due to the beam applied to one packet of atoms. Data in (b) are fit to a functional form $N(t) / N=\exp \left[-\left(\Omega^{2} \Gamma^{\prime} t\right) /\left(\Gamma^{\prime 2}+4 \Delta^{2}\right)\right]$ to determine the Rabi frequency $\Omega$ and broadened linewidth $\Gamma^{\prime}$. Here $\Delta$ is the detuning and $t=4 \mu \mathrm{s}$ is the duration of the laser pulse. The fit, shown as the solid curve, gives $\Omega=2 \pi \times 1.1 \mathrm{MHz}$ and $\Gamma^{\prime}=2 \pi \times 10.0 \mathrm{MHz}$. These values are used in a two-level model to calculate the solid curve in (a). (c) Visibility of the measured interferometer curve for various detunings of the Stark beam. The visibility is non-zero even when the atom loss is large. 


\subsubsection{Measurement of the ac Stark shift near resonance}

Using a similar technique as the one described above, we used the interferometer to measure the ac Stark shift directly at the $5 P_{3 / 2}, F=3$ atomic resonance. The dispersion shape of the energy shift through resonance is well known, and can be measured using microwave spectroscopy of the ground state hyperfine transition [106]. However, an atom interferometric technique would be necessary for atoms that lack ground state hyperfine structure. A measurement for a BoseEinstein condensate is of particular interest due to the possibility for collective line broadening effects $[91,92]$.

In the resonant ac Stark shift measurement, we read out a phase shift caused by the Stark beam at a fixed time and intensity. By changing the phase $\theta$ of the standing wave during the recombination pulse, the fraction of atoms that remain at rest varies as $\cos ^{2}(\phi / 2+\theta)$, where $\phi$ is the interferometer phase from Eq. (4.30). We therefore take an interference curve by stepping through $\theta$ while keeping the Stark beam fixed. The phase shift of this curve gives $\phi$, which we measured for various detunings of the Stark beam. The results are shown in Fig. 4.4(a), with error bars from the fits of the interference curves. The overall offset of $-0.3 \mathrm{rad}$ is consistent with the residual phase induced by the guide itself with no Stark beam. We are not entirely sure how this offset comes about, and have observed a slow change in the offset over time.

On resonance, photon scattering cannot be neglected. In our case, if an atom absorbs a photon, it leaves the condensate fraction and is lost from the magnetic trap. To avoid losing all the atoms, a relatively low intensity beam $\left(I \approx 40 \mu \mathrm{W} / \mathrm{cm}^{2}\right)$ is used that is applied for a short duration $(4 \mu \mathrm{s})$ during the first reflect pulse. The small number of photons makes measuring the intensity with the camera and power meters difficult. Instead, we determine the intensity by measuring the atom loss when the experiment is performed without applying the recombination pulse. In this case, we observe just two packets, one of which has been exposed to the Stark beam and one that has not. From the ratio of the number of atoms in the packets we can accurately determine the atom loss, and thus the scattering rate. The results are shown in Fig.4.4(b), along with a comparison to a calculation for a two-level system with an added decay term, which has 
a Hamiltonian [107]

$$
H=\hbar\left(\begin{array}{cc}
0 & \Omega / 2 \\
\Omega / 2 & \Delta-i \Gamma / 2
\end{array}\right)
$$

We adjusted the Rabi frequency $\Omega$ in the calculation to match the atom loss observed in the experiment. To obtain good agreement, however, we have found that a broadened linewidth of $\Gamma^{\prime}=2 \pi \times(10.0 \pm 0.9) \mathrm{MHz}$ is required, 1.6 times larger than the natural linewidth $\Gamma$. The solid curve in Fig. 4.4(a) shows the phase shift calculated with these parameters, which also exhibits the same broadening. The broadened linewidth also appears when estimating the beam intensity from the Rabi frequency in the calculation using Eq. (3.21). Using the brodened linewidth gives a value which agrees with the measured intensity $I \approx 40 \mu \mathrm{W} / \mathrm{cm}^{2}$, whereas using the natural linewidth gives a value about three times higher.

Since atoms are lost from the packet to which the Stark beam is applied, it is expected that a differential phase shift $\Delta \phi$ accumulates between the two packets due to the different chemical potential $\Delta \phi \approx \Delta \mu t / \hbar$. We do not observe this effect in the experiment, however. A possible explanation for this is the fact that for a condensate, the uncertainty in the measurement of the number of atoms is quite high. Extracting the absolute number through a fit to a Gaussian as in Sec. 2.1.4 is not necessarily a good measure due to the high optical density and the fact that the atomic distribution is actually a Thomas-Fermi distribution. Alternatively, getting the number of atoms from the measured chemical potential depends strongly on the packet size, which is limited by our imaging resolution. Since the chemical potential depends on the initial number of atoms, the uncertainty in it will likewise be high. However, for the differential phase shift to be non-observable, the initial number of atoms would have to be around 100 times smaller than what is measured.

\subsubsection{Collective effects}

We confirmed the existence of this broadening by measuring the loss of atoms from a plain condensate after application of a laser beam with different detunings, both in and out of the guide 


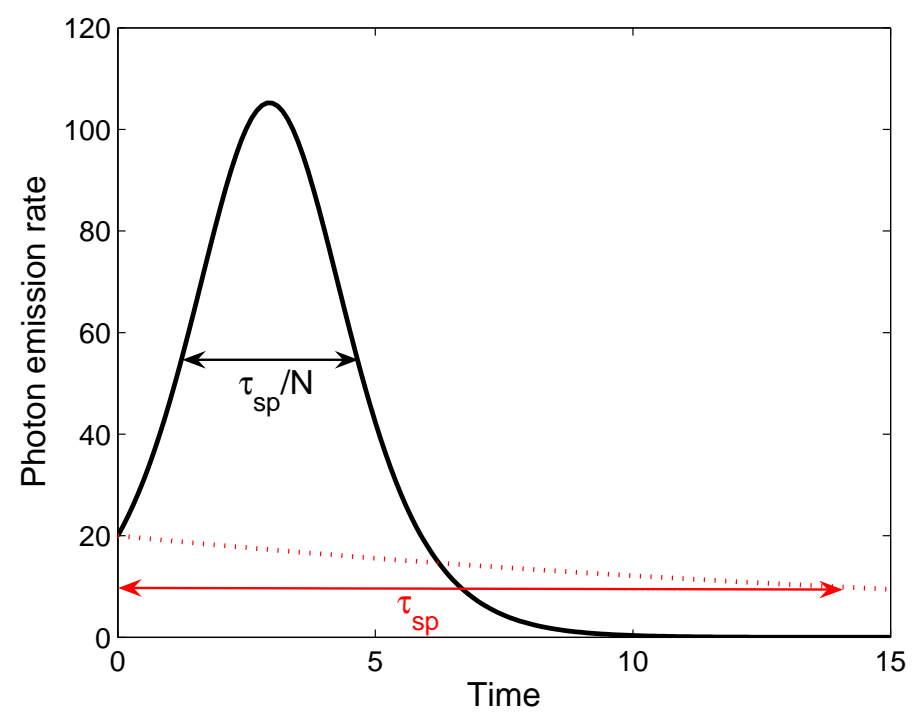

Figure 4.5: Schematic of light radiated from a collection of atoms. For a set of $N$ independent atoms, spontaneous emission has an exponentially decaying intensity (red dotted curve). For a correlated ensemble of $N$ atoms, superradiant emission is shorter and more intense, with a time constant reduced by a factor of $N$ versus the independent case. The shown situation of the peak emission occurring after the initial excitation is only valid for incoherent excitation of all the atoms into the excited state. In our case, a subset of the atoms is initially excited coherently, leading to the peak emission occurring at time zero. The decay nevertheless occurs with a time constant $\tau_{\mathrm{sp}} / N$ and the emission is increased.

fields. In all cases, the beam intensity was low enough that conventional power broadening was negligible.

We attribute the broadening effect both to superradiant scattering $[108,109]$ and multiple photon scattering. Superradiance has been predicted previously for condensates $[91,92]$ and observed for off-resonant light [110]. It occurs when the radiation of atoms is influenced by the presence of other atoms. This happens when the density of atoms is high or the atoms are illuminated by an intense light pulse. In this case, the atoms radiate faster and more intensely than a similar collection of independent atoms, as can be seen in Fig. 4.5, An explanation is that the optical dipoles of the different atoms become correlated, leading to the emission of light into a collective mode. 
The predicted linewidth is $[91,109]$

$$
\Gamma^{\prime}=\frac{3}{2} N\left(\frac{\lambda}{2 \pi L}\right)^{2} \Gamma
$$

for a condensate of size $L$. If we use $L=\left(L_{x} L_{y} L_{z}\right)^{1 / 3} \approx 22 \mu \mathrm{m}$ for our Thomas-Fermi widths $L_{i}$, we obtain $\Gamma^{\prime}=1.5 \Gamma$, in reasonable agreement with the observations. However, the broadening effect is observed only for optically thick clouds, typically with resonant optical densities of 2 to 3. It is therefore also possible that multiple photon scattering plays a role.

Superradiance depends strongly on the polarization of the incident light. Since the atomic cloud acts like a gain medium for the light, the effect will be largest along the longest axis of the cloud [110]. However, we are unable to measure this effect, since in our setup the rotating bias field is always perpendicular to the waveguide. Therefore, when using light polarized along the guide axis, there is always a superposition of $\sigma^{+}$and $\sigma^{-}$light. Due to the Zeeman shifts in the guide, the atoms only interact with one of these two components. To measure the polarization dependence, we would need $\pi$-light polarized both along the guide axis and perpendicular to it, which is not possible in our experimental setup.

We have also performed a more detailed investigation into the broadening in our experiment. Linewidths were measured by illuminating the atoms with a weak scatter beam while in the waveguide, then imaging the atoms after around $100 \mathrm{~ms}$ wait time to get the number of atoms remaining. This was performed for different confinement strengths in the waveguide and for different intensities of the scatter beam, as seen in Fig. 4.6. We observe a strong intensity dependence in the measured linewidths, as well as dependence on the atomic density. For noncondensed clouds with low optical density, the unbroadened linewidth is recovered. We believe that the intensity dependence comes from the effects of multiple photon scattering.

Though multiple scattering is quite complicated to describe theoretically [111], we can use a simple one dimensional model to qualitatively describe what its effect on the atom loss will be. In this picture, light does not penetrate into the cloud of atoms very far when the light is tuned 


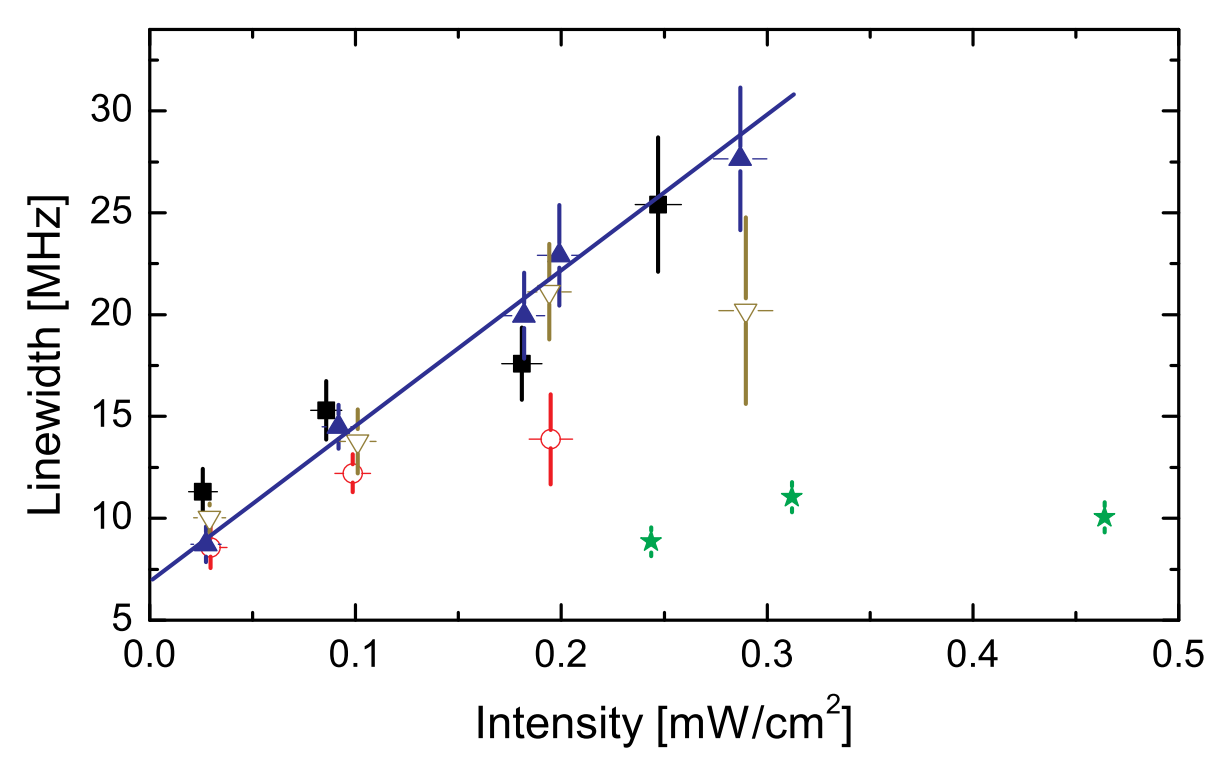

Figure 4.6: Atom loss linewidths for various trap configurations. Black solid squares (ם) are in a $4.1 \mathrm{G}$ bias field waveguide, and blue solid triangles $(\mathbf{\Lambda})$ are in a $20.5 \mathrm{G}$ bias field waveguide. Red circles ( $($ ) and olive inverse triangles $(\nabla)$ are also in a $20.5 \mathrm{G}$ bias field waveguide, the former for a non-condensed cloud of atoms, the latter for a condensate with about half as many atoms as before, obtained by evaporating further. Green stars $(\star)$ are from absorption linewidth measurements in the $20.5 \mathrm{G}$ bias field waveguide. The solid line is a fit to the $20.5 \mathrm{G}$, regular evaporation data.

on resonance, meaning that not as many atoms as expected are lost. However, when the laser is detuned from resonance, light can penetrate further, and more atoms are lost, meaning that the measured loss curve looks broader.

In this one dimensional model, the gas of atoms is divided into $N$ layers, as seen in Fig.4.7. In each layer, $A_{n}$ denotes the forward propagating intensity and $B_{n}$ the backwards propagating intensity. The probability for a photon to scatter at any given layer is $2 p$, where $p$ is the probability to either scatter forwards or backwards. The intensities in adjacent layers can then be related as

$$
\begin{aligned}
A_{n+1} & =(1-p) A_{n}+p B_{n+1} \\
B_{n} & =(1-p) B_{n+1}+p A_{n},
\end{aligned}
$$




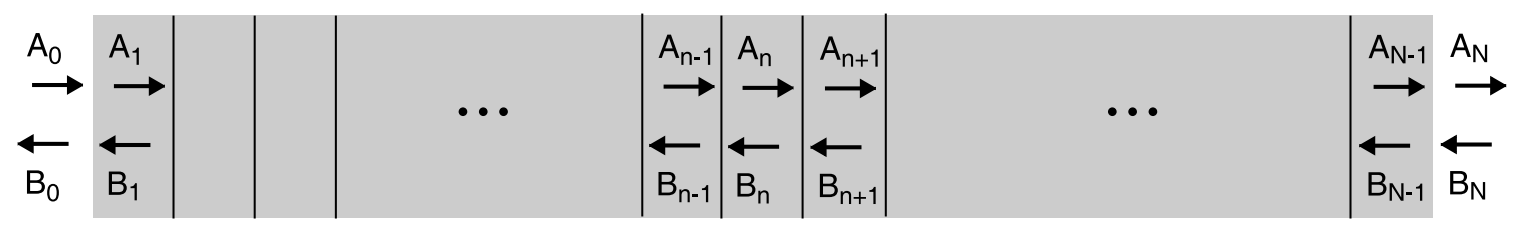

Figure 4.7: Schematic of the one dimensional model for multiple photon scattering. The gas of atoms is divided into $N$ layers. In each layer, $A_{n}$ denotes the forward propagating intensity and $B_{n}$ the backwards propagating intensity.

which can be expressed in matrix form as

$$
\left(\begin{array}{c}
A_{n} \\
B_{n}
\end{array}\right)=\frac{1}{1-p}\left(\begin{array}{cc}
1 & -p \\
p & 1-2 p
\end{array}\right)\left(\begin{array}{c}
A_{n+1} \\
B_{n+1}
\end{array}\right) \equiv M\left(\begin{array}{c}
A_{n+1} \\
B_{n+1}
\end{array}\right) .
$$

By induction, it can be shown that $n$ applications of the matrix $M$ give

$$
M^{n}=\frac{1}{1-p}\left(\begin{array}{cc}
1+(n-1) p & -n p \\
n p & 1-(n+1) p
\end{array}\right)
$$

and the inverse

$$
M^{-n}=\frac{1}{1-p}\left(\begin{array}{cc}
1-(n+1) p & n p \\
-n p & 1+(n-1) p
\end{array}\right) \text {. }
$$

So by considering the intensities after exiting the gas and using the boundary condition $B_{N}=0$, we can express $A_{N}$ and $B_{0}$ in terms of the known incident intensity $A_{0}$ as

$$
\begin{gathered}
A_{N}=A_{0} \frac{1-p}{1+(N-1) p} \\
B_{0}=A_{0} \frac{N p}{1+(N-1) p} .
\end{gathered}
$$

The intensities in each layer $n$ can then be expressed as

$$
\left(\begin{array}{c}
A_{n} \\
B_{n}
\end{array}\right)=\frac{A_{0}}{1+(N-1) p}\left(\begin{array}{c}
1+(N-n-1) p \\
(N-n) p
\end{array}\right)
$$


from which the total intensity in the layer $n$ can be calculated to be

$$
I_{n}=A_{n}+B_{n}=A_{0} \frac{1+2\left(N-n-\frac{1}{2}\right) p}{1+(N-1) p} .
$$

We can now relate this intensity to the quantities observable in the experiment. The transmission $t$ is the probability for a photon to pass through the cloud of atoms without scattering, and is related to the absorption $\alpha$ through $t=(1-2 p)^{N}=e^{-\alpha}$. For large $N$, we can then express the scatter probability $p$ as $p=\alpha / 2 N$. Also, the position within the cloud $z$ can be expressed in terms of its thickness $L$ by $z=n L / N$. With these observables, the intensity at any position can be expressed in terms of $z, \alpha$, and the incident intensity $I_{\text {inc }}$. In the limit of large $N$, the intensity can be written as

$$
I(z)=I_{\mathrm{inc}} \frac{1+\alpha(1-z / L)}{1+\alpha / 2}
$$

The intensity can now be used in order to calculate the atom loss. For beam intensities much less than the saturation intensity, the light scattering rate $R$ is proportional to the intensity, $R(z)=$ $\beta I(z)$, where $\beta=\left(\Gamma / 2 I_{\text {sat }}\right) \Gamma^{2} /\left(\Gamma^{2}+4 \Delta^{2}\right)$. In terms of the total atom number $N$ and the constant number density $n=N / L$, the fraction of atoms remaining can then be calculated as

$$
\begin{aligned}
\frac{N_{\text {remain }}}{N} & =\frac{1}{N} \int_{0}^{L} n e^{-R(z) t} d z \\
& =e^{-\beta I_{\text {inc }} t} \frac{\sinh Q}{Q},
\end{aligned}
$$

where $Q=\beta I_{\text {inc }} t \alpha /(2+\alpha)$.

A fit of this function to the exponential of a Lorentzian, as above, shows very good agreement. This model qualitatively gives correct results, giving a linewidth which is dependent on the incident intensity and the optical density of the cloud. However, the amount of broadening is less than that seen in the experiment, which leads us to conclude that our model is imperfect. Also, the three dimensional nature of the problem is important in that much of the light scattered from the atoms is scattered into directions not necessarily parallel to the incident light, and away from 
the cloud of atoms. A more complicated model will therefore be needed to fully describe these effects.

In addition to the linewidth measurements using atom loss, as described above, we also took absorption linewidths of the condensate for various beam intensities, also seen in Fig.4.6. In this case, we measure the photon loss instead of the atom loss. As seen in the figure, the absorption linewidth seems to be independent of the probe intensity, with an average value larger than the natural linewidth. This complicates the interpretation of the broadening of the dispersion curve in the measurement of the phase shift, since the constant value of the absorption linewidths and the low intensity values of the linewidth of the atom loss measurements both agree with the broadened width of the dispersion curve. At this point, we therefore do not know which of these two effects causes the dispersion curve broadening.

The possible presence of collective line broadening has important implications. It means that the phase shift imposed by a near-resonant Stark beam depends on $N$, which thus provides a source of controllable non-linearity for the quantum state of the atoms. For instance, condensate interferometers are expected to be limited by the fact that the atoms interact [77]. The number of atoms in a packet has unavoidable quantum fluctuations, and thus the interaction energy is uncertain. This in turn imparts an uncertain phase shift that limits the coherence time of the interferometer [65]. However, if a near-resonant Stark beam can provide a number-dependent phase shift, this could be used to correct for the unknown interaction phase and thus recover the coherence time. This would be beneficial even if it required the loss of some atoms due to the light scattering. More generally, any source of nonlinearity can produce squeezed quantum states, which are useful for applications in quantum information [112].

In conclusion, we have used a guided-wave atom interferometer to make high-quality measurements of the ac Stark shift. The method is unique in providing good accuracy, high sensitivity, and operation at arbitrary frequency. The polarizability measured at large detunings was found to agree with theoretical calculations with the experimental accuracy. Measurements at the atomic resonance, however, exhibited a broadened linewidth that we attribute to superra- 
diant scattering, and which may permit non-linear control of the condensate phase. We are investigating this possibility further, and also plan to extend our measurements to the dc polarizability by inserting one of the packets in between a pair of well-calibrated electric field plates. Through experiments such as these, guided-wave interferometers are beginning to make important contributions to metrology. 
Solange etwas ist, ist es nicht das, was es gewesen

sein wird.

Martin Walser

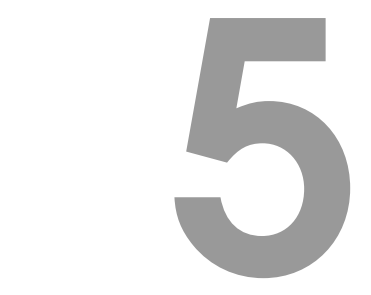

Conclusion

After showing the potential of guided-wave atom interferometers with the measurement of the ac Stark shift, we aim to further improve our apparatus and perform more measurements. A first improvement is the reduction of the confinement potential along the waveguide axis. As shown in Sec. 3.3.3, the confining potential causes phase gradients to form on the packets of atoms in the interferometer, limiting the interaction times in the interferometer. Reducing these phase gradients should improve interferometer times and arm separations over what has already been achieved.

Since the confinement along the guide comes largely from the leads of the waveguide structure, an obvious solution is simply to extend the guide to make it longer. Since the current guide structure is located inside the vacuum chamber, changing it out is not straightforward, as it would involve breaking vacuum and replacing the glass vacuum chamber. Nevertheless, this is a step that is being planned. The guide will then consist of a similar four-wire setup, but will be external to a vacuum chamber that is flatter than the one currently in use. Even with a flatter chamber, the current-carrying rods will be further from the atoms than in the current setup inside the vacuum chamber. However, since the current-carrying elements are not in the vacuum, dispersing the heat from an increase in current should not be too difficult. 


\subsection{Outlook: Measuring static polarizabilities}

One of the next measurements to be performed with our atom interferometer is a measurement of the static polarizability of ${ }^{87} \mathrm{Rb}$. The energy shift from an electric field $E$ is given by

$$
U=-\frac{1}{2} \alpha\left\langle E^{2}\right\rangle
$$

where $\alpha$ is the polarizability. This parameter impacts most interactions of atoms with electric fields, and appears in expressions for the index of refraction, electron scattering, van der Waals interactions, Rayleigh scattering and the Casimir-Polder effect [113]. For ${ }^{87} \mathrm{Rb}$, the currently accepted best value dates back to 1974 and has a $2 \%$ error [114]. Recent measurements of the polarizabilities in other alkali atoms have achieved relative uncertainties of $0.7 \%$ in $\mathrm{Li}$ [68], $0.3 \%$ in $\mathrm{Na}$ [44] and $0.15 \%$ in Cs [115]. With our apparatus, a precision of $10^{-3}$ should be possible, and with some improvements, getting to $10^{-4}$ precision is conceivable.

The polarizability is of particular interest to research on atomic clocks. Atomic clocks are currently used as a frequency standard, and the SI definition of a second is related to the frequency of the ground state hyperfine transition of Cs. However, Rb atomic clocks have some advantages in performance at lower temperatures and in their compactness [116], leading to their adoption as a secondary standard and their use on GPS satellites [117].

Light shifts in general must be well understood when building an atomic clock [118]. At the precisions that these clocks have reached, shifts of the atomic energy levels from black-body radiation become important as well [119]. These cause a temperature-dependent shift of the hyperfine energy states. Since the definition of the second is given for a temperature of $0 \mathrm{~K}$, whereas atomic clocks are usually operated at room temperature, this black-body shift must be calculated to extrapolate from the measurement to the $0 \mathrm{~K}$ value of the frequency. While the shift itself is related to the polarizability of the hyperfine states, ab initio calculations can be performed for the black-body shift which involve some assumptions about the atomic structure. To check the accuracy of these calculations, the static polarizability can be calculated and compared to exper- 


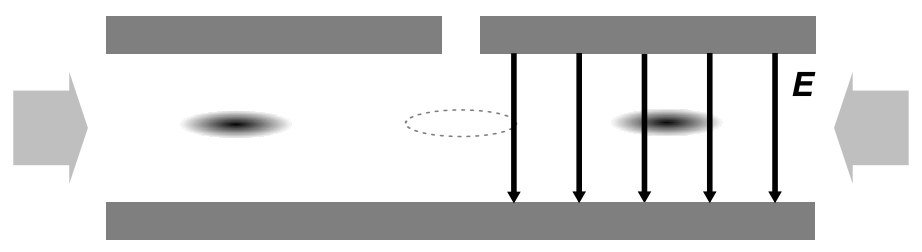

Figure 5.1: Schematic of the measurement of the static polarizability. The condensate is placed between the plates of a parallel plate capacitor. After splitting the condensate into two packets, an electric field is applied to one of the packets. The interferometer sequence is then completed as usual and the phase shift from the field measured.

imental values [120]. This gives an estimate of the uncertainty of the calculated results. A more accurate value of the static polarizability of ${ }^{87} \mathrm{Rb}$ would therefore allow for better comparison to these calculations and more precise ${ }^{87} \mathrm{Rb}$ atomic clocks.

The recent measurements of the static polarizability in $\mathrm{Na}$ and Li were both performed with an atomic beam interferometer. The two arms of the interferometer were passed on either side of a grounded septum, and an electric field was applied to one arm but not the other. Using Eq. (5.1), the phase acquired during an interaction time $\tau$ of the atoms with the electric field is then

$$
\phi=\frac{\alpha E^{2} \tau}{2 \hbar}
$$

The accuracy of these measurements was largely limited by uncertainties in the calibration of the electric field $E$ as well as the interaction time $\tau$.

A schematic of the setup in our experiment can be seen in Fig. [5.1, The whole experiment is performed inside a device similar to a parallel plate capacitor. One side of the capacitor is connected to a voltage $V_{0}$, whereas the other side is grounded. An electric field can therefore be applied to one arm but not the other. At the maximum arm separation, the electric field is turned on for a time $\tau$, which is precisely known. The packets then traverse the rest of the trajectory, and the output is measured as explained previously.

The electric field can be determined precisely by knowing the applied voltage $V_{0}$ and the plate spacing $d$. For a plate spacing of $d=100 \mu \mathrm{m}$ and an interaction time of $\tau=1 \mathrm{~ms}$, a $20 \mathrm{~V}$ 
applied voltage gives an acquired phase of $10^{3} \mathrm{rad}$. Measuring this phase to $0.1 \mathrm{rad}$ corresponds to the $10^{-4}$ accuracy that we ultimately wish to achieve. An applied voltage $V_{0}=20 \mathrm{~V}$ can easily be measured with a precise voltmeter, and the finite rise and fall times of the voltage can be accounted for.

The plate spacing is not so easily measured. However, we can take advantage of the geometry of the capacitor setup and use the gold coated glass plates as a Fabry-Perot etalon. For a spacing of $d=100 \mu \mathrm{m}$, the free spectral range is $v_{\mathrm{FSR}}=1.5 \times 10^{12} \mathrm{~Hz}$. A thin gold coating on the plates of $25 \mathrm{~nm}$ gives a reflectivity of $R=85 \%$ and a transmissivity of $T=10 \%$ for light with a wavelength of $800 \mathrm{~nm}$. The finesse of the etalon is then $\mathscr{F}=\pi \sqrt{R} /(1-R)=20$, which gives a resonant linewidth $\delta v=c / 2 \mathscr{F} d=7.5 \times 10^{10} \mathrm{~Hz}$. The center of a resonance line can typically be located to within $5 \%$ of its width. The precision with which the center of the line can be located is therefore $8 \mathrm{GHz}$, or about $0.25 \%$ of $v_{\mathrm{FSR}}$. By measuring the positions of two of these lines separated by about 25 free spectral ranges, the plate spacing can then be measured with the desired precision of $10^{-4}$. In the near infrared, this corresponds to a change in wavelength of around $75 \mathrm{~nm}$. The Ti:Sapphire laser we use for most of the experiment has a range of around $100 \mathrm{~nm}$, so this could be used. Alternatively, a free running laser diode at $690 \mathrm{~nm}$ or $850 \mathrm{~nm}$ could be used to supplement this.

Using this method, the entire plate can be illuminated and spatial variations in the plate spacing can be seen. Furthermore, the calibration can be performed after the plates have been put into the vacuum chamber and rechecked as necessary, so that drifts over time or due to the bake-out procedure can be accounted for.

The large arm separation achievable with our interferometer means that the curvature of the electric field at the position of the atoms when the field is applied is small. Any curvature of the electric field will lead to a spatial variation of the energy shift. This causes a phase gradient across the cloud, leading to a loss of visibility, as discussed previously. As calculated in Appendix $\mathrm{D}$ the electric field at large separations can be approximated by

$$
|\mathbf{E}|=E_{0} e^{-\pi|y| / d}
$$


The phase difference across a condensate of length $L$ can then be estimated as

$$
\Delta \phi \approx \frac{\partial \phi}{\partial E} \frac{d E}{d y} L \approx \frac{2 \pi L}{d} \phi e^{-\pi|y| / d}
$$

Plugging in the values for a $T=72 \mathrm{~ms}$ symmetric double-sided interferometer, where we see a visibility of $50 \%, y=210 \mu \mathrm{m}$ and $d=100 \mu \mathrm{m}$ gives $\Delta \phi / \phi=5 \times 10^{-3}$. Keeping $\Delta \phi$ below $1 \mathrm{rad}$ therefore requires keeping $\phi$ below $200 \mathrm{rad}$. Though this is significantly less than the target of $10^{3} \mathrm{rad}$, Fig.4.4(a) indicates that we can measure $\phi$ with 0.05 rad uncertainty, leading to a relative uncertainty in $\alpha$ of $2.5 \times 10^{-4}$. This is only slightly higher than what we are aiming for, and a reduction could be achieved either by extending the arm separation or by stabilizing the output in order to reduce the measurement uncertainty in $\phi$.

The capacitor is currently being made in cooperation with the electrical engineering department. A schematic of the capacitor design can be found in Appendix D When performing this experiment, the capacitor will be mounted inside the vacuum chamber. After making a condensate, the atoms must therefore be translated into the capacitor using a magnetic field. The interferometer experiment will then be performed between the plates of the capacitor. Since the imaging must then be performed through the plates of the capacitor, the frequency of the imaging beam must be tuned near one of the resonances of the Fabry-Perot etalon. This will allow the probe light to be transmitted through the etalon, and in fact will increase the absorption signal.

\subsection{Summary}

The main goals of my dissertation research were to implement and characterize an atom interferometer using Bose-Einstein condensates and then use it in a practical application. In this sense, the main results of this dissertation will be recapitulated in this section.

The aspect of the research on which most time and energy was spent was by far the successful implementation of a condensate interferometer. After first achieving Bose-Einstein condensation and subsequently loading the condensate into a novel waveguide (Chapter2), we were able 
to achieve long interaction times of up to $72 \mathrm{~ms}$ and large arm separations of up to $0.5 \mathrm{~mm}$ with our interferometer with a stable phase of the output (Sec. 3.2). With a fluctuating phase, the achieved interference times were even longer, up to about $1 \mathrm{~s} \mathrm{(Sec.3.3.3.3).}$

Since starting work on our interferometer, several other cold atom interferometers using guiding potentials have been realized [121]. Soon after reporting our initial results, a group at MIT announced a $200 \mathrm{~ms}$ coherence time using a BEC on a chip split by using radio frequency radiation to go from an initial harmonic trap to a double well potential [65]. This long coherence time, much longer than the predicted phase diffusion time for their setup, was attributed to number squeezing due to atomic interactions during the separation. While the long coherence time in this experiment is impressive, the spatial separation of the arms is only $8.7 \mu \mathrm{m}$, and the number squeezing increases the quantum uncertainty of the phase, limiting its usefulness for practical measurements.

Two groups have recently demonstrated a similar free-oscillation interferometer to the one described in Sec. 3.3.3.3. An experiment at JILA yielded a similar fluctuating phase as in our experiment in a tighter confining trap with an interferometer time $T=160 \mathrm{~ms}$ [83], and an experiment in Tokyo was carried out yielding a fluctuating phase at $T=100 \mathrm{~ms}$ [84]. In this case, the group was able to vary the confining potential and found a stable phase for $T=58 \mathrm{~ms}$.

An interferometer using non-condensed cold atoms was realized at Harvard, which was used to demonstrate a Sagnac interferometer with sufficient sensitivity to measure the rotation of the earth [122]. This experiment achieved a coherence time of $T=50 \mathrm{~ms}$, though this interferometer is significantly more complex than the one described in this dissertation.

The next major result of this dissertation is the characterization of the interferometer, as performed in Sec. 3.3, The description of phase gradients from the confining potential as the major limiting factor to our interferometer performance, as well as the list of possible noise sources serve to stimulate discussion about future improvements to the device. The obvious step of a weaker confinement potential should be implemented in the next generation interferometer device in our lab. 
The final major result is the measurement of the ac Stark effect. While the potential of condensates in interferometry was realized soon after their first experimental achievement, this experiment proves the practical applicability of guided wave condensate interferometers. While performing these measurements, we noticed a broadened linewidth both in the measurement of the phase and of the atom number, as seen in Chapter 4 , We attribute this broadening to multiple photon scattering and superradiant scattering, which is a collective effect in light scattering. This has an important implication, since a method to achieve number-dependent phase shifts could be used to correct for unknown interaction phases and extend the coherence times of interferometers. More investigation into this subject is necessary, and is currently underway.

The future of cold atom interferometers, both in general and at the University of Virginia, looks bright. With the achieved sensitivity, we are able to competitively measure the static polarizability of ${ }^{87} \mathrm{Rb}$, as described earlier in this chapter. After some improvements to the experimental apparatus, further interesting experiments are conceivable. An obvious choice would be to realize a ring geometry waveguide, with which rotations could be measured. Taking advantage of the large arm separations achievable in our interferometer, one could also think of passing one arm inside a high-finesse cavity and using the phase shift from the cavity field in order to nondestructively measure the number of photons in the cavity. Another possibility would be to pass one arm close to a material surface and measure the phase shift from the Casimir-Polder force. Through such experiments, guided wave atom interferometers can become a useful tool both for basic research and for industrial applications. 


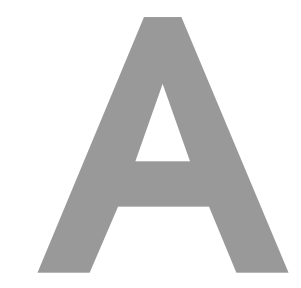

Rubidium

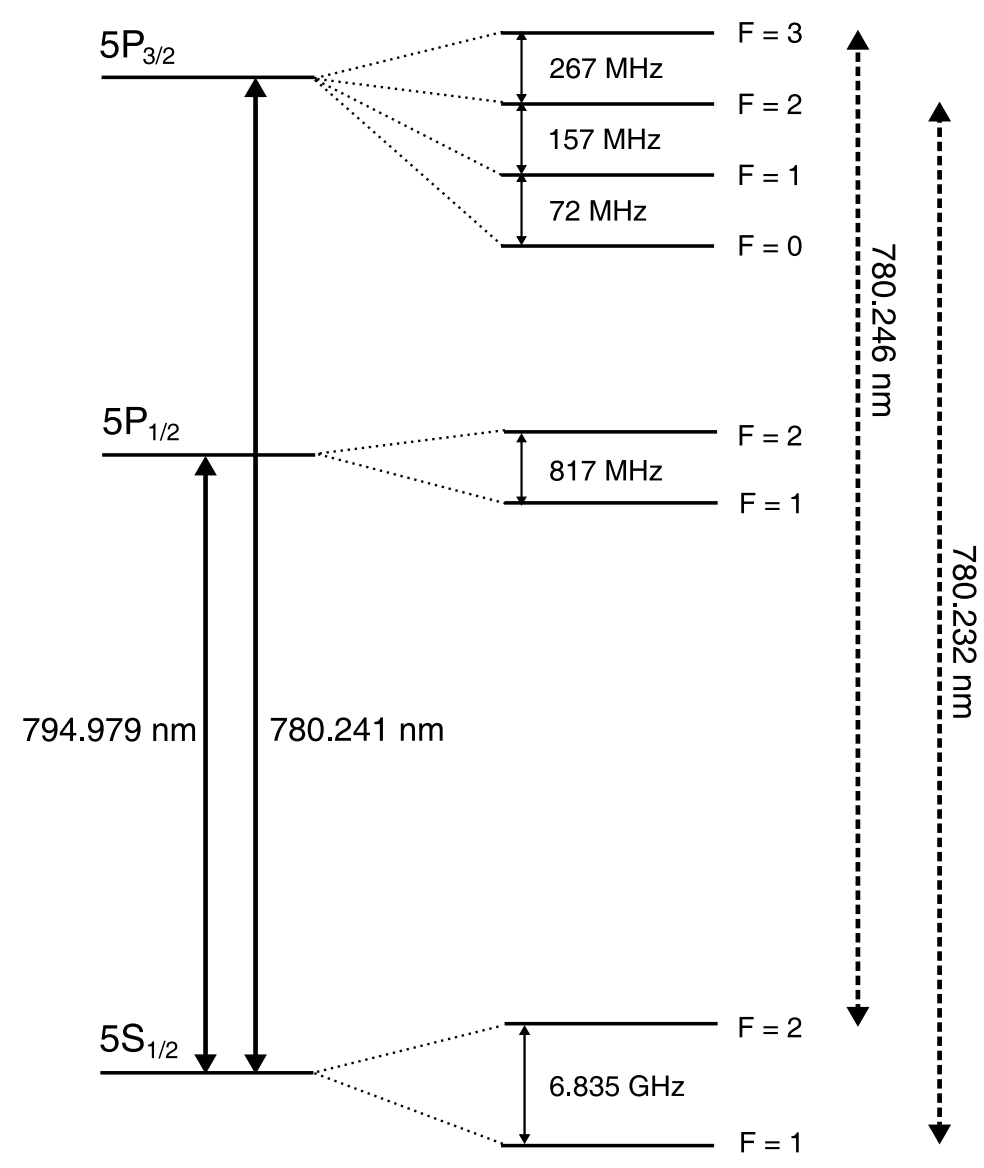

Figure A.1: ${ }^{87} \mathrm{Rb}$ principal transitions with hyperfine structure. Light at $780.246 \mathrm{~nm}$ is used for the MOT transitions, whereas $780.232 \mathrm{~nm}$ light is used for "repumping" atoms that have fallen into the $F=1$ ground state. 
(a) Fundamental constants

\begin{tabular}{lcc}
\hline \hline Name & Symbol & Value \\
\hline Speed of light & $c$ & $2.99792458 \times 10^{8} \mathrm{~m} / \mathrm{s}$ \\
Standard gravity & $g$ & $9.80665 \mathrm{~m} / \mathrm{s}^{2}$ \\
Permeability of vacuum & $\mu_{0}$ & $4 \pi \times 10^{-7} \mathrm{~N} / \mathrm{A}^{2}$ \\
Permittivity of vacuum & $\epsilon_{0}$ & $8.854187817 \times 10^{-12} \mathrm{~F} / \mathrm{m}$ \\
Planck's constant & $h$ & $6.62606876 \times 10^{-34} \mathrm{Js}$ \\
& $\hbar=h / 2 \pi$ & $1.054571596 \times 10^{-34} \mathrm{Js}$ \\
Elementary charge & $e$ & $1.602176462 \times 10^{-19} \mathrm{C}$ \\
Bohr magneton & $\mu_{B}$ & $9.27400899 \times 10^{-24} \mathrm{~J} / \mathrm{T}$ \\
Atomic mass unit & $\mathrm{u}$ & $1.66053873 \times 10^{-27} \mathrm{~kg}$ \\
Electron mass & $m_{e}$ & $9.10938188 \times 10^{-31} \mathrm{~kg}$ \\
Bohr radius & $a_{0}$ & $0.5291772083 \times 10^{-10} \mathrm{~m}$ \\
Boltzmann's constant & $k_{B}$ & $1.3806503 \times 10^{-23} \mathrm{~J} / \mathrm{K}$ \\
\hline \hline
\end{tabular}

(b) Properties of ${ }^{87} \mathrm{Rb}$

\begin{tabular}{lcc}
\hline \hline Name & Symbol & Value \\
\hline Atomic mass & $m$ & $86.909180520 \mathrm{u}$ \\
& & $1.44316060 \times 10^{-25} \mathrm{~kg}$ \\
Nuclear spin & $I$ & $3 / 2$ \\
Scattering length & $a$ & $5.77 \mathrm{~nm}$ \\
\hline \hline
\end{tabular}

(c) Transition properties

\begin{tabular}{lcc}
\hline \hline Name & Symbol & Value \\
\hline Frequency & $\omega_{o}$ & $2 \pi \cdot 384.2304844685 \mathrm{THz}$ \\
Wavelength (vacuum) & $\lambda$ & $780.241209686 \mathrm{~nm}$ \\
Lifetime & $\tau$ & $26.24 \mathrm{~ns}$ \\
Natural linewidth & $\Gamma$ & $2 \pi \cdot 6.065 \mathrm{MHz}$ \\
Recoil velocity & $v_{r}$ & $5.8845 \mathrm{~mm} / \mathrm{s}$ \\
Recoil temperature & $T_{r}$ & $361.96 \mathrm{nK}$ \\
Doppler temperature & $T_{D}$ & $146 \mu \mathrm{K}$ \\
\hline Electron spin g-factor & $g_{S}$ & 2.0023193043737 \\
Electron orbital g-factor & $g_{L}$ & 0.99999369 \\
Fine structure & $g_{J}\left(5^{2} S_{1 / 2}\right)$ & 2.00233113 \\
Landé g-factor & $g_{J}\left(5^{2} P_{1 / 2}\right)$ & 0.666 \\
Nuclear g-factor & $g_{J}\left(5^{2} P_{3 / 2}\right)$ & 1.3362 \\
\hline \hline & $g_{I}$ & -0.0009951414 \\
\hline \hline
\end{tabular}

Table A.1: Constants relevant to the experiment. (a) Fundamental constants. (b) Properties of ${ }^{87} \mathrm{Rb}$. (c) $5^{2} \mathrm{~S}_{1 / 2} \rightarrow 5^{2} \mathrm{P}_{3 / 2}$ transition properties 


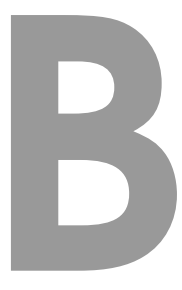

\section{Canceling the waveguide potential along the} guide

As shown in Sec. 3.3.3, phase gradients due to a confining potential limit the interaction times in the interferometer. Since the component of the confining potential in the waveguide comes from the finite size of the guide and the effect of the waveguide leads, one possibility would be to make the guide longer. Since the waveguide structure is inside the vacuum chamber, however, this is rather difficult without breaking vacuum and designing a new vacuum chamber. Another possibility is to try to cancel out the confining potential with additional current elements. A calculation of what current elements could be used will be given in this appendix.

We can consider a four-wire setup as show in Fig. B.1. Two wires separated by a distance

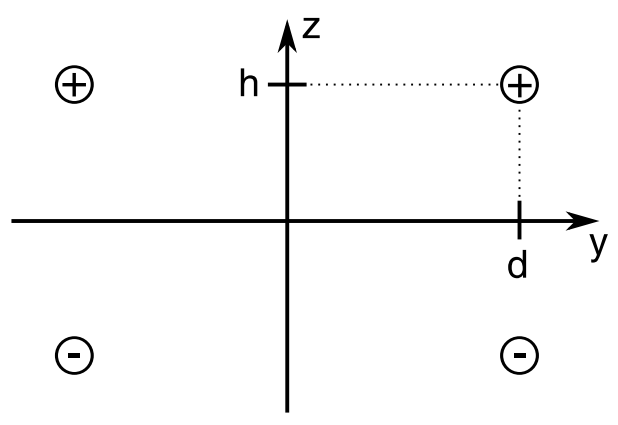

Figure B.1: Schematic of the positions of the four-wire potential cancellation. Two wires, separated by a distance $2 d$ are located a distance $h$ above the cloud of atoms located at the origin. Two wires with equal and opposite current are located the same distance below the atoms. 
$2 d$ run along the $x$-axis a distance $h$ above the atoms, and back the same distance below the atoms. In the case of very long wires, there will be no component along the $x$-axis, and the two other components are simply found using the Biot-Savart law. Using an oscillating current $I=I_{0} \cos \Omega t$, the field is calculated as

$$
\begin{aligned}
& B_{y}=\frac{\mu_{0} I}{2 \pi}\left[-\frac{z-h}{(y-d)^{2}+(z-h)^{2}}-\frac{z-h}{(y+d)^{2}+(z-h)^{2}}+\frac{z+h}{(y-d)^{2}+(z+h)^{2}}+\frac{z+h}{(y+d)^{2}+(z+h)^{2}}\right] \\
& B_{z}=\frac{\mu_{0} I}{2 \pi}\left[\frac{y-d}{(y-d)^{2}+(z-h)^{2}}+\frac{y+d}{(y+d)^{2}+(z-h)^{2}}-\frac{y-d}{(y-d)^{2}+(z+h)^{2}}-\frac{y+d}{(y+d)^{2}+(z+h)^{2}}\right] .
\end{aligned}
$$

We are interested in the potential at $z=0$, for which we find

$$
B=B_{y}=\frac{\mu_{0} I}{\pi}\left[\frac{h}{(y-d)^{2}+h^{2}}+\frac{h}{(y+d)^{2}+h^{2}}\right] .
$$

This can be expanded to second order in $y$ as

$$
B \approx \frac{\mu_{0} I}{\pi}\left[\frac{2 h}{d^{2}+h^{2}}+\frac{12 h d^{2}-4 h^{3}}{\left(d^{2}+h^{2}\right)^{3}} y^{2}\right] \equiv\left[B_{2}+\kappa y^{2}\right] \cos \Omega t .
$$

We now need to time-average this field with the fields generated by the bias field (2.7) and waveguide quadrupole (2.25). Doing this and expanding to second order in the position, we find

$$
\begin{aligned}
\left|\mathbf{B}_{\mathrm{tot}}\right| \approx B_{0}[ & 1+\frac{B_{1}^{\prime 2}}{2 B_{0}}\left(x^{2}+z^{2}\right) \cos ^{2} \Omega t+\frac{B_{1}^{\prime}}{B_{0}}\left(x \sin \Omega t \cos \Omega t-z \cos ^{2} \Omega t\right)+\frac{B_{2}^{2}}{2 B_{0}^{2}} \cos ^{2} \Omega t \\
& \left.+\frac{B_{2} \kappa}{B_{0}} y^{2} \cos ^{2} \Omega t-\frac{B_{1}^{\prime 2}}{2 B_{0}^{2}}\left(x^{2} \sin ^{2} \Omega t \cos ^{2} \Omega t-2 x z \cos ^{3} \Omega t \sin \Omega t+z^{2} \cos ^{4} \Omega t\right)\right] .
\end{aligned}
$$

Taking the time average then gives a time averaged field

$$
\left\langle\left|\mathbf{B}_{\text {tot }}\right|\right\rangle=B_{0}+\frac{B_{1}^{\prime 2}}{16 B_{0}}\left(3 x^{2}+z^{2}\right)-\frac{B_{1}^{\prime}}{2 B_{0}} z+\frac{B_{2}^{2}}{4 B_{0}}+\frac{B_{2} \kappa}{2 B_{0}} y^{2},
$$


(a) Anti-Trapping Frequency for $2 \mathrm{~d}=1 \mathrm{~cm}, \mathrm{~B}_{0}=20 \mathrm{G}$

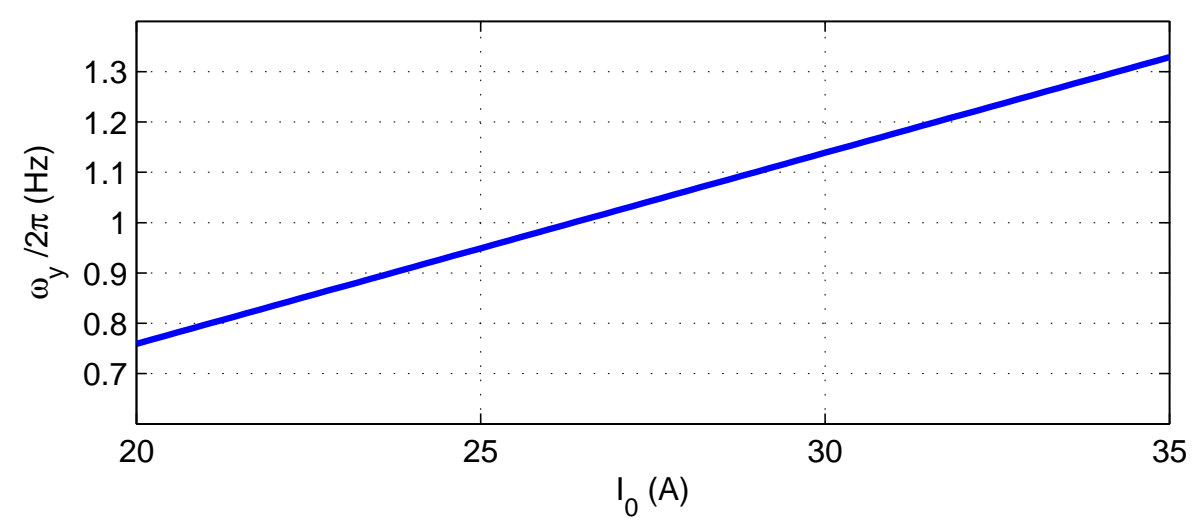

(b) Anti-Trapping Frequency for $b=30 \mathrm{~A}, \mathrm{~B}_{0}=20 \mathrm{G}$

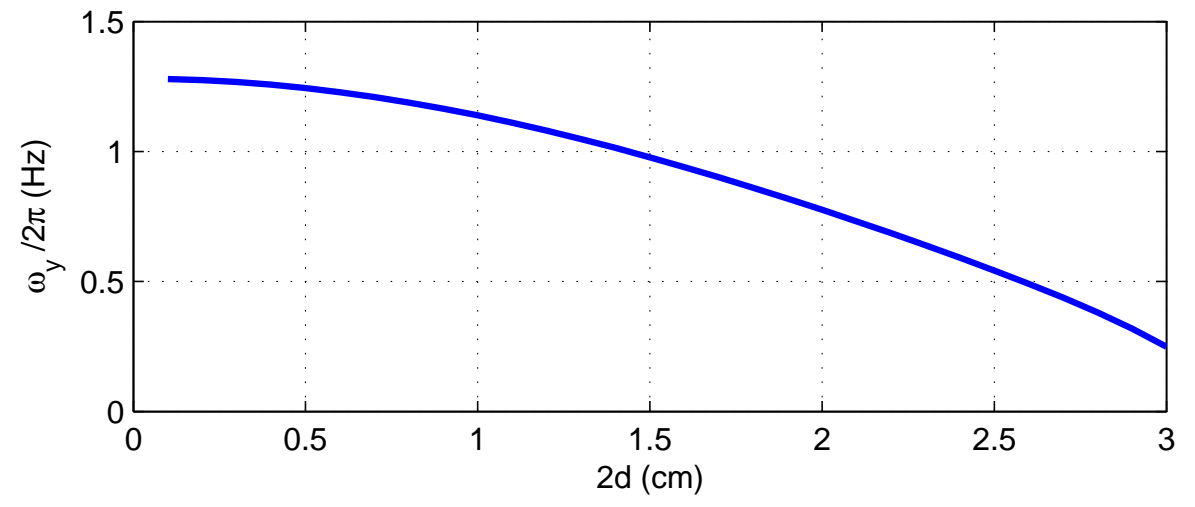

(c) Anti-Trapping Frequency for $b=30 \mathrm{~A}, 2 \mathrm{~d}=1 \mathrm{~cm}$

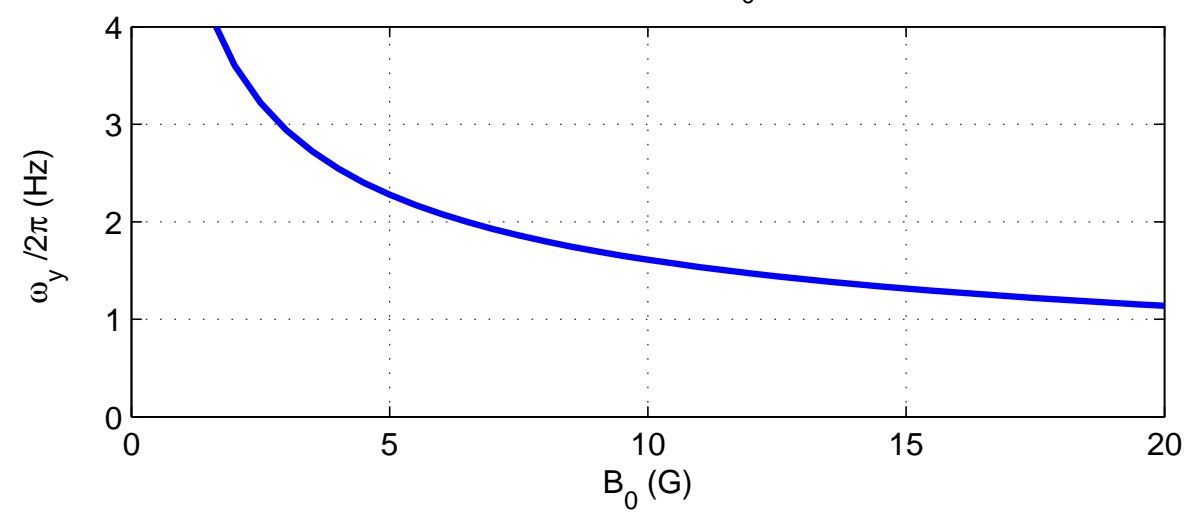

Figure B.2: Calculation of anti-trapping potential frequency for various parameters. (a) Changing the current $I_{0}$, (b) varying the separation of the wires (nominally $1 \mathrm{~cm}$ ), and (c) changing the bias field strength. 
from which we find the anti-trapping frequency

$$
\omega_{y}=-2 \sqrt{\frac{2 \mu}{m B_{0}}} \mu_{0} I_{0} \frac{h}{\left(d^{2}+h^{2}\right)^{2}} \sqrt{2 d^{2}-h^{2}} .
$$

We want to compensate for a $1.2 \mathrm{~Hz}$ trapping potential at a bias field of $B_{0}=20 \mathrm{G}$. For the chamber used in the experiment, the vertical distance $h$ is always fixed at $5.5 \mathrm{~cm}$. As we can see in Fig. B.2 a cancellation can then be achieved at a peak current of around $30 \mathrm{~A}$ when the wires are separated horizontally by $2 d=1 \mathrm{~cm}$. As seen in Fig. B.3, this increases the trapping potential in the $z$ direction slightly.

One concern with such a setup is its mechanical stability. In such a weak trap, any gradients of the potential along the guide direction will cause the cloud of atoms to move significantly. Such fluctuating gradients can be caused by a differential motion of the trap structure and the wires generating the anti-trapping potential. Similarly, any high frequency mechanical noise would cause fluctuations in the trapping potential, leading to the types of phase noise described in Sec. 3.3.4. These considerations have led us to the conclusion that designing a longer guide structure is superior to an external cancellation of the confining potential. 

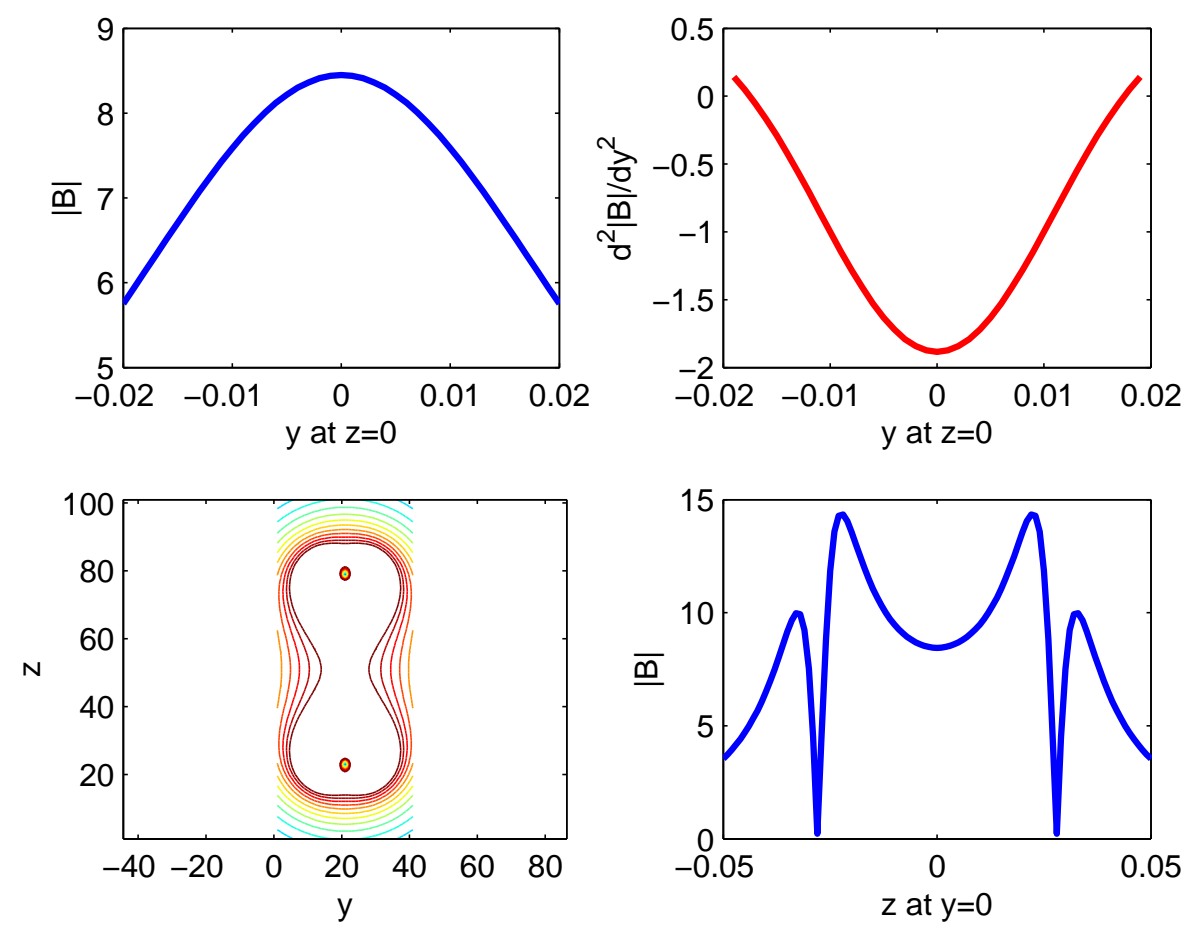

Figure B.3: Calculations of the anti-trapping potential for $I_{0}=30 \mathrm{~A}, 2 d=1 \mathrm{~cm}$, and $B_{0}=20 \mathrm{G}$. 


\section{C}

\section{Switching circuit}

Before loading the atoms into the waveguide, we need to be able to turn off the external quadrupole quickly enough so that the cloud of atoms does not expand too far or falls under the influence of gravity. These influences would disturb the cloud and prevent the extraction of useful information from an image of the cloud. For imaging, the velocity information of the cloud of atoms is used to determine temperatures and derived parameters. When switching the external quadrupole field off, non-adiabatic processes will preserve this information. The field should therefore turn off on a much faster timescale than the period of trap oscillations.

A problem arises from extremely fast switching times when using the high currents that are needed for magnetic trapping. A quickly changing magnetic field gives a large voltage according to the law of induction $V=-L(d I / d t)$, where $L$ is the inductance and the magnetic field is proportional to the current $I$. According to Lenz's rule, this voltage works to keep the magnetic field from decreasing when switching off and from increasing when turning on. The large expected voltage can also damage components in the circuit. The inductance of the coils used in our experiment was measured to be $50 \mu \mathrm{H}$.

We have therefore designed a circuit that provides fast switching times of less than $1 \mathrm{~ms}$ and the ability to control the strength of the magnetic trap. This circuit is described in detail in Ref. [123]. Several modifications have been performed since that time, which will be outlined here.

Fig. C.1 shows an overview of the main circuit, consisting of a current supply which can 


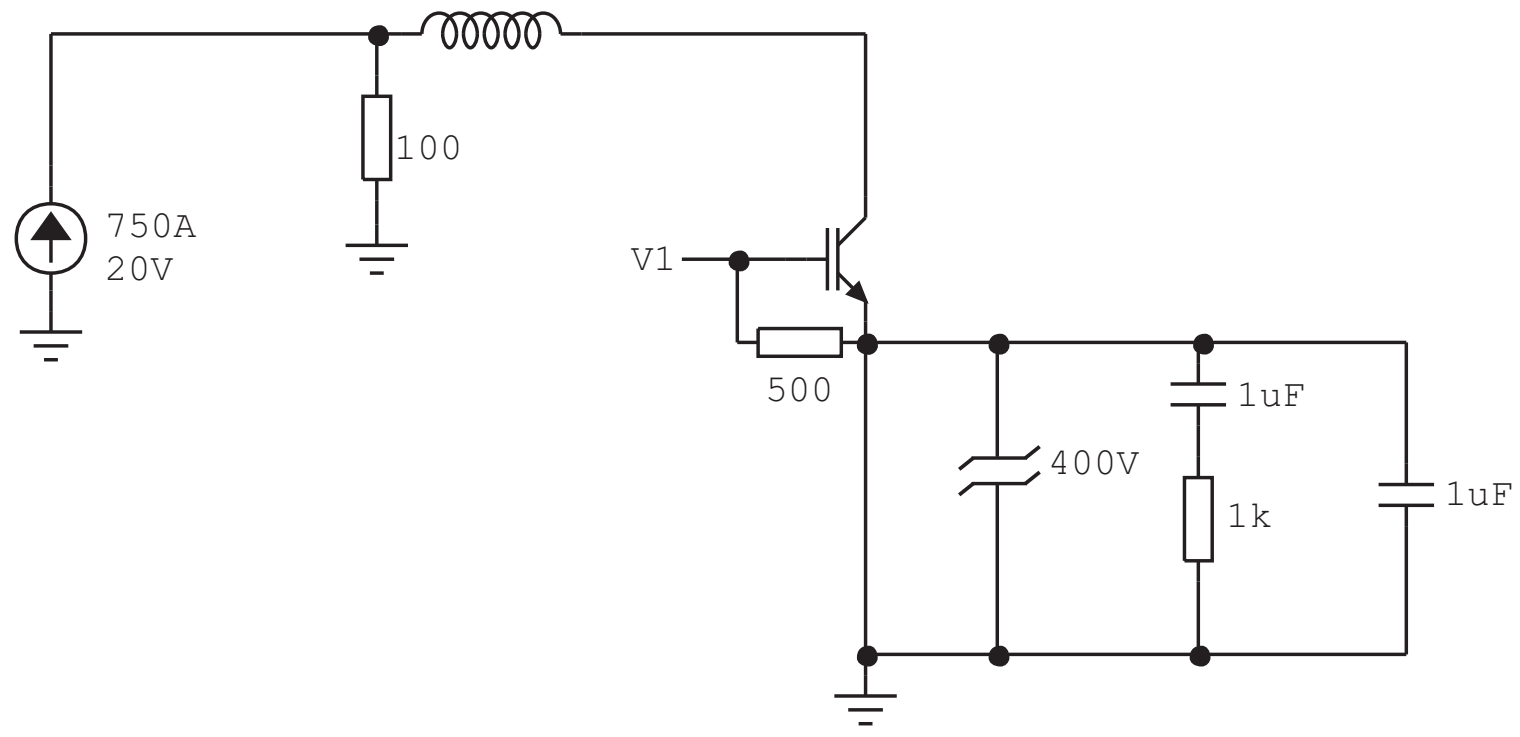

Figure C.1: Schematic of the main switching circuit. The current supply provides up to $750 \mathrm{~A}$ of current through the coils, which is switched using three IGBTs. A varistor, as well as capacitors and resistors, limit the voltage when switching the current off.

deliver up to $750 \mathrm{~A}$ at $20 \mathrm{~V}$, the coils generating the external quadrupole field, and a set of 3 insulated-gate bipolar transistors (IGBTs) which function as a high-power switch. In addition, there are varistors, capacitors and resistors parallel to the IGBTs to limit the voltage.

Fig. C.2 shows the control circuit for the IGBTs. This circuit converts a 0/5 V digital CMOS input into a $0 / 15 \mathrm{~V}$ output with enough current capacity to control the gate of the IGBTs.

One problem that was encountered was that when IGBTs were somehow accidentally turned on halfway, e.g. through a gate voltage in between 0 and $15 \mathrm{~V}$, while large amounts of current were flowing, they would overheat and become shorted. At a price of nearly $\$ 200$ per IGBT, this is something that should be avoided whenever possible. We therefore incorporated a voltage interlock into the circuit which shuts everything off when the $\pm 15 \mathrm{~V}$ from the power supply drop below a set value of around $\pm 12 \mathrm{~V}$. The interlock circuit is shown in Fig.C.3

Finally, Table C.1 gives the pin connections on the circuit board which holds the switching control and voltage interlock circuits. 


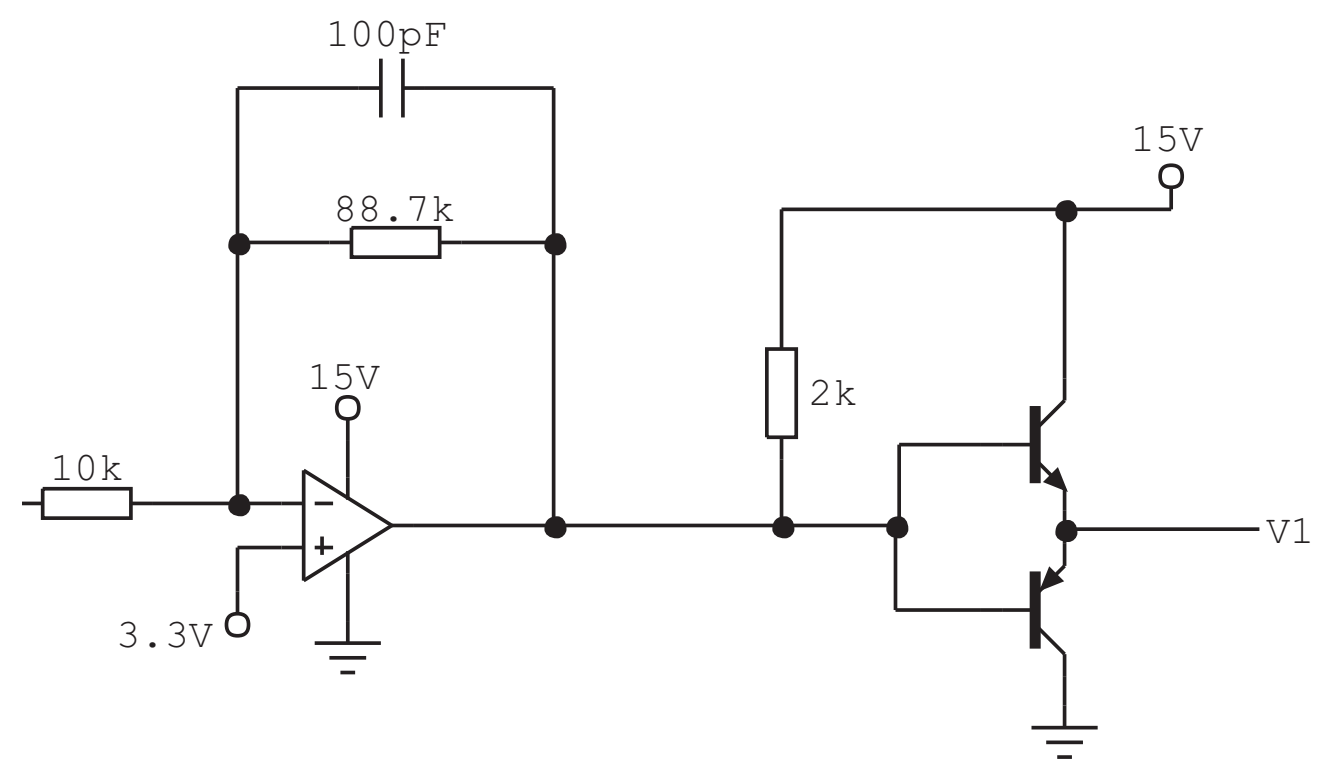

Figure C.2: Schematic of the control circuit. A 0/5 V digital signal (ON/OFF) is converted to a $0 / 15 \mathrm{~V}$ signal, and enough current can be sourced to provide the gate voltage to the IGBTs.

\begin{tabular}{lcc}
\hline \hline Pin & Description & Color \\
\hline $\mathrm{A}, \mathrm{Z}$ & ground & green \\
2 & $+15 \mathrm{~V}$ & red \\
6 & $V_{1}$ (output for IGBT) & orange \\
11 & $V_{\text {control }}($ common) & yellow \\
12 & $V_{\text {control }}$ (signal) & orange \\
13 & $V_{\text {analog }}($ common) & white \\
14 & $V_{\text {analog }}($ signal) & gray \\
18 & ON/OFF & purple \\
21 & $-15 \mathrm{~V}$ & black \\
\hline
\end{tabular}

Table C.1: Pin connections on Vector card for switching circuit. 


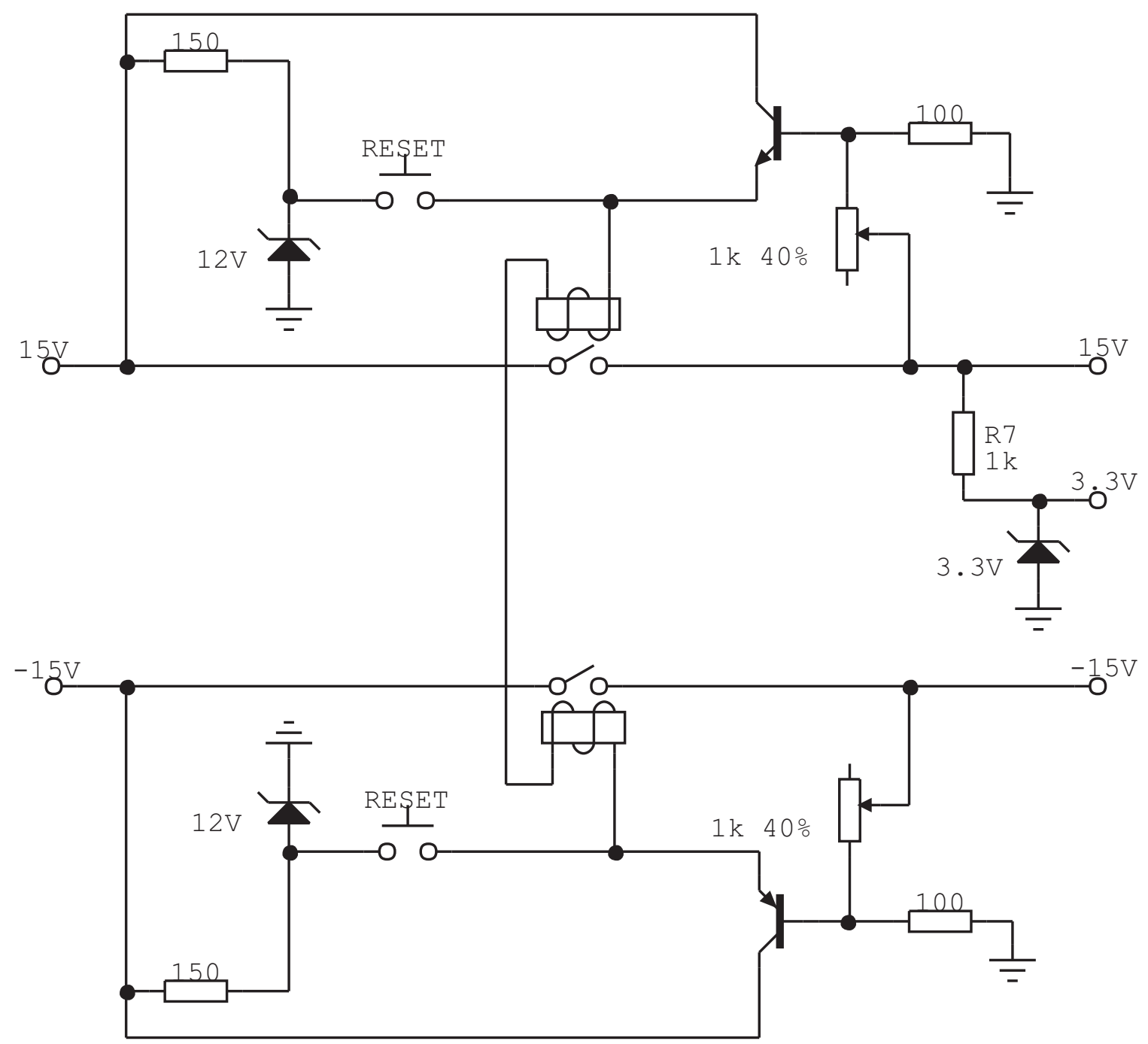

Figure C.3: Schematic of the power supply interlock circuit. When the $\pm 15 \mathrm{~V}$ fall below a set value of $\pm 12 \mathrm{~V}$ (this value can be regulated using the variable resistor), the output voltages become zero, causing the current to stop flowing. This circuit is meant to protect the IGBTs. 


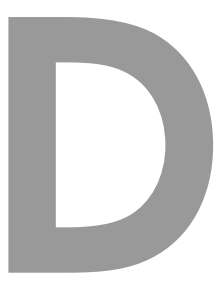

\section{Capacitor design}

In designing a device for the measurement of the static polarizability of ${ }^{87} \mathrm{Rb}$, we need a constant electric field which is applied to one arm of the interferometer, whereas the other arm experiences no field. In addition, the curvature of the field should be as small as possible near the position of the atoms when the field is turned on, so that small variations in the position of the atoms do not affect the phase, and phase gradients across the cloud are held to a minimum.

The design is basically a parallel plate capacitor with plate separation $t$, in which one of the plates is divided into a grounded half and a charge-carrying half, as seen in Fig.D.1. We consider first an infinite capacitor and try to find the potential $\phi$ for all $x$ and all $0<y<t$.

The boundary conditions in this case are $\phi=0$ for $y=0$ and $\phi=V_{0} H(x)$ for $y=t$, where $H(x)$ is the Heaviside function. Starting with a separation of variables, we find $\phi(x, y)=X(x) \cdot Y(y)$,

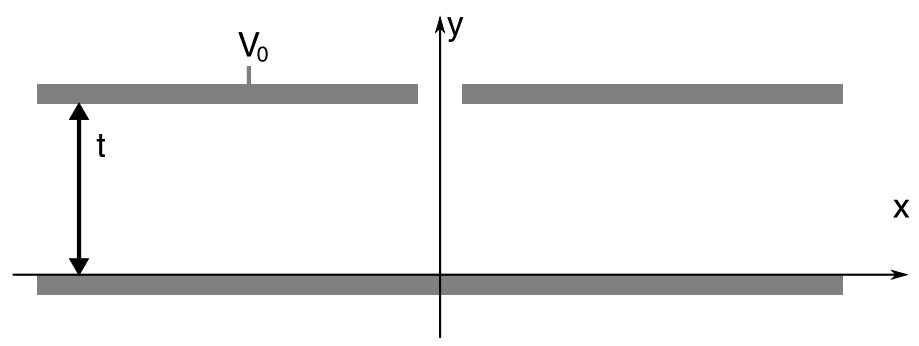

Figure D.1: Schematic of the parallel plate capacitor. The two plates are separated by a distance $t$. A voltage $V_{0}$ is applied to one half of the top plate; the other half, as well as the bottom plate are grounded. 
with $X=e^{i k x}$ and $Y=\sinh k y$. A superposition of these solutions gives us

$$
\phi(x, y)=\int_{-\infty}^{\infty} d k A_{k} e^{i k x} \sinh k y
$$

with the boundary condition

$$
\phi(x, t)=V_{0} H(x)=\int_{-\infty}^{\infty} d k A_{k} e^{i k x} \sinh k t \equiv \int_{-\infty}^{\infty} \frac{d k}{2 \pi} F(k) e^{i k x}
$$

This gives us the coefficient as

$$
A_{k}=\frac{1}{2 \pi} \frac{1}{\sinh k t} F(k)
$$

Performing the Fourier transform of $V_{0} H(x)$ then gives $F(k)$ as

$$
F(k)=\left(-\frac{1}{i k}+\pi \delta(k)\right) V_{0}
$$

Using this and the fact that $\lim _{k \rightarrow 0} \sinh k y / \sinh k y=\lim _{k \rightarrow 0}(y \cosh k y) /(t \cosh k t)=y / t$, we can express the potential as

$$
\phi(x, y)=\frac{V_{0} y}{2 t}-\frac{V_{0}}{2 \pi i} \int_{-\infty}^{\infty} d k \frac{e^{i k x}}{k} \frac{\sinh k y}{\sinh k t}
$$

We can now proceed in two ways: either do the integral numerically, or convert the integral into an infinite sum using the residue theorem. In the first case, the integral can be put into a form

$$
\phi(x, y)=\frac{V_{0} y}{2 t}-V_{0} \int_{0}^{\infty} \frac{d k}{\pi} \frac{\sin k x}{k} \frac{\sinh k y}{\sinh k t}
$$

which can then be solved numerically. Fig. D.2(a) shows the numerical calculation of the electric field at $y=t / 2$ for a plate spacing of $100 \mu \mathrm{m}$.

In the other case, we find that when doing the integral, there are an infinite number of poles on the imaginary axis. The poles are located at $k=-i n \pi / t$, for integer $n$. The residue at these 

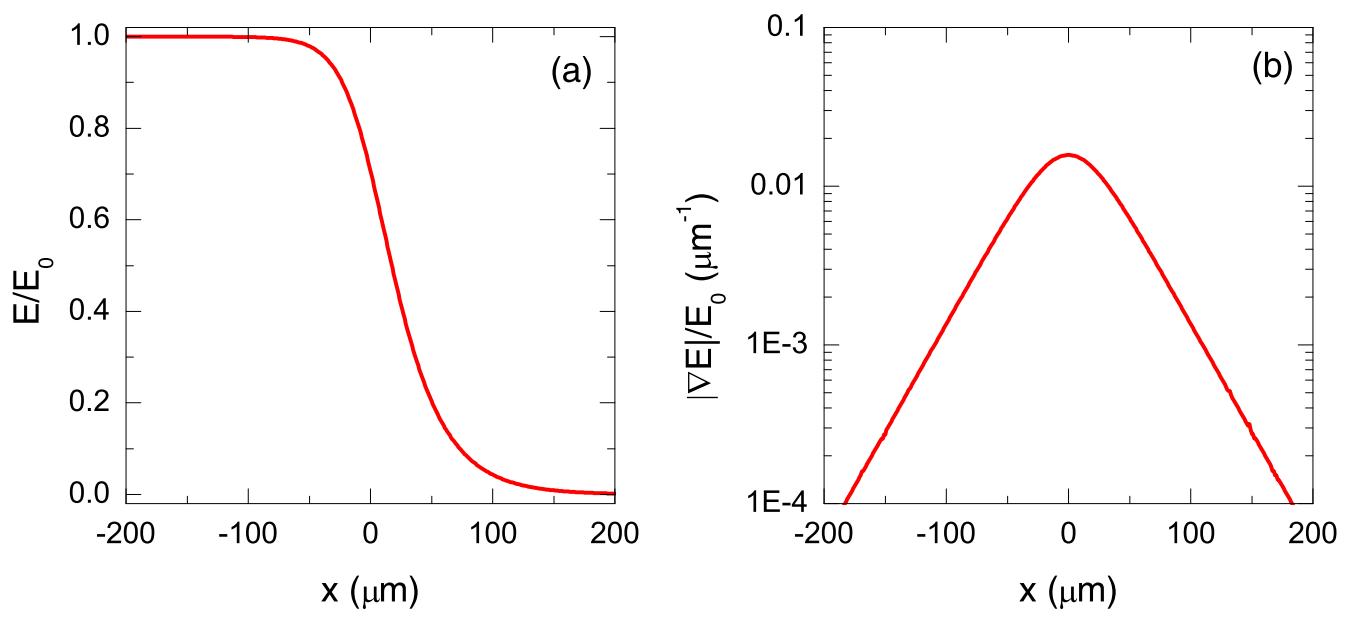

Figure D.2: (a) Numerical calculation of the electric field halfway in between the two plates of the capacitor for a plate spacing of $100 \mu \mathrm{m}$. (b) Calculation of the gradient of the electric field for the same parameters.

poles can easily be calculated as

$$
\operatorname{Res}_{-i n \pi / t}=(-1)^{n} \frac{t}{n \pi} e^{-n \pi|x| / t} \sin \frac{n \pi y}{t}
$$

and the residue at zero is found to be $y / t$, giving an expression for the potential in the form of an infinite sum

$$
\phi(x, y)=\left\{\begin{array}{c}
0 \\
V_{0} y / t
\end{array}\right\} \pm \frac{V_{0}}{\pi} \sum_{n=1}^{\infty} \frac{e^{-n \pi|x| / t}}{n} \sin \frac{n \pi y}{t} \text { for }\left\{\begin{array}{c}
x>0 \\
x<0
\end{array}\right\}
$$

For $x \gg 0$, only the $n=1$ term in the sum contributes a significant amount, so we keep only this term. Also, to simplify things, we look only at positive $x$, since the curvature should be symmetric in $x$. The potential in this case is

$$
\phi(x, y) \approx-\frac{V_{0}}{\pi} e^{-\pi x / t} \sin \frac{\pi y}{t},
$$



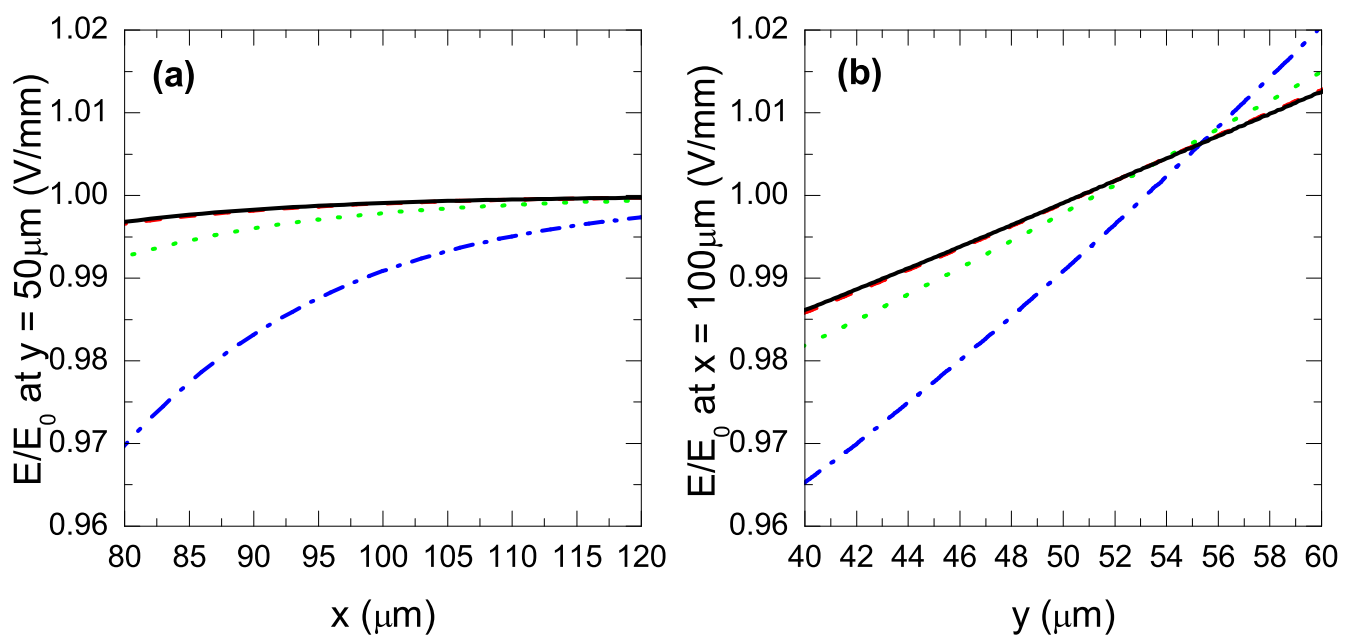

Figure D.3: Electric field for various gap sizes at an atom separation of $200 \mu \mathrm{m}$ (a) halfway between the capacitor plates $(y=50 \mu \mathrm{m})$ and (b) at the maximum separation $(x=100 \mu \mathrm{m})$. In both graphs, the black solid curve is with no gap between the electrodes, the red dashed curve a gap of $10 \mu \mathrm{m}$, the green dotted curve $50 \mu \mathrm{m}$, and the blue dash-dotted curve $100 \mu \mathrm{m}$.

and the electric field can be calculated as the magnitude of the gradient of the potential as

$$
|\mathbf{E}|=\frac{V_{0}}{t} e^{-\pi x / t}
$$

The magnitude of the gradient of the electric field then gives the curvature of the potential as

$$
|\nabla| \mathbf{E}||=\frac{V_{0} \pi}{t^{2}} e^{-\pi x / t}
$$

In order for the electric field to be constant for all spacings $t$, we choose $V_{0}=E_{0} t$ with $E_{0}=$ $1 / 100 \mathrm{~V} / \mu \mathrm{m}$. The curvature is then shown in Fig. D.2(b).

The results of the numerical calculations were confirmed using finite element analysis. This also allowed us to model the effects of a finite sized capacitor, which showed that edge effects are negligible for capacitor plate sizes as small as $1 \mathrm{~mm}$. With this model, it was also possible to calculate the gradient of the electric field for various gap sizes between the two electrodes on the upper plate of the capacitor, as seen in Fig. D.3. For a $50 \mu \mathrm{m}$ gap, the gradient can be estimated as 
$|\nabla E| / E_{0}=9 \times 10^{-4} \mathrm{~V} / \mu \mathrm{m}^{2}$ in the $x$-direction and $|\nabla E| / E_{0}=1.5 \times 10^{-3} \mathrm{~V} / \mu \mathrm{m}^{2}$ in the $y$-direction, which is only slightly more than the curvature shown in Fig.D.2(b).

(a)

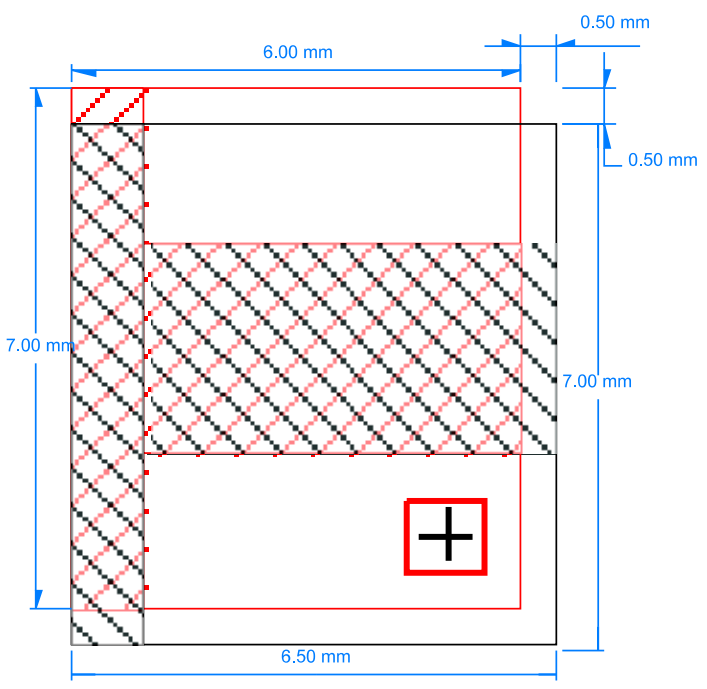

(b)

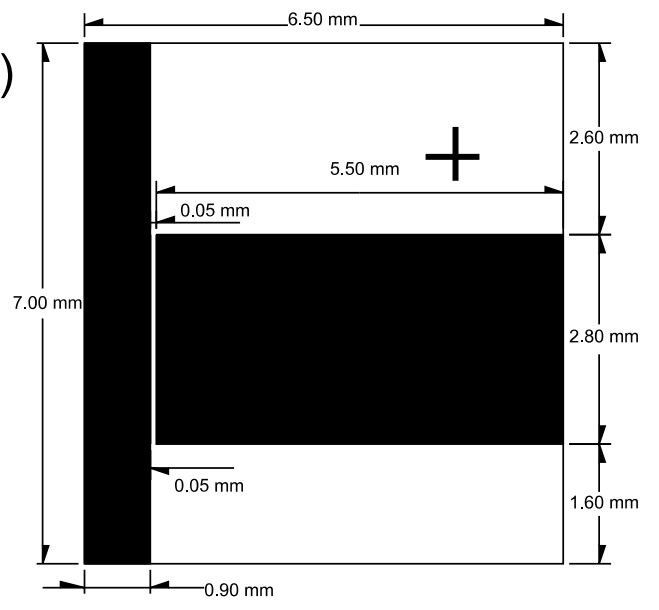

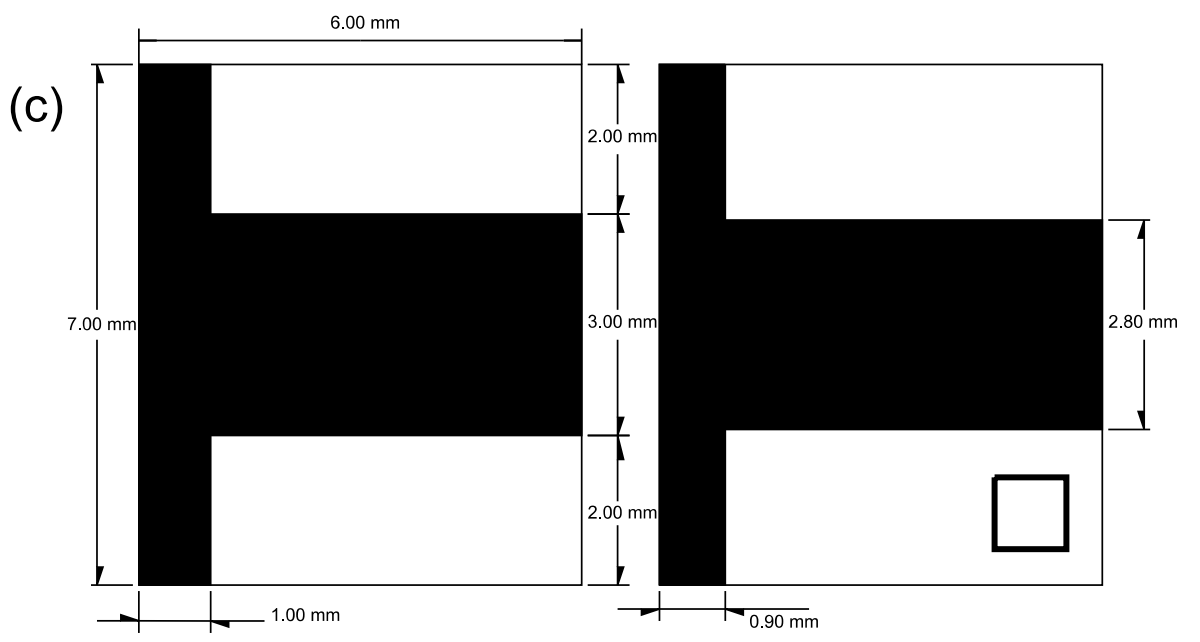

Figure D.4: Parallel plate capacitor specifications. (a) Diagram of how the two plates fit together. The black outline is for the bottom piece, while the red outline is the top piece. The two pieces are slightly offset so that electric connections can be made to the edges of the gold-plated parts. (b) Schematic of the top piece. The black area is coated with a $25 \mathrm{~nm}$ layer of gold. The cross is for alignment purposes. (c) Schematic of the bottom piece. On the left is the mask for etching the glass, where the black area is etched $100 \mu \mathrm{m}$ deep. On the right is the mask for coating with gold (black area). The coating is again $25 \mathrm{~nm}$, and the square is for alignment with the cross of the top piece. 


\section{Bibliography}

[1] S. Bose. "Plancks Gesetz und Lichtquantenhypothese." Zeitschriftf. Physik 26, 178 (1924).

[2] A. Einstein. "Quantentheorie des einatomigen idealen Gases. Zweite Abhandlung." Sitzungsber. Preuss. Akad. Wiss. 1, 3 (1925).

[3] K. Huang. Statistical Mechanics. John Wiley \& Sons (1987).

[4] F. Dalfovo, S. Giorgini, L. P. Pitaevskii, and S. Stringari. "Theory of Bose-Einstein condensation in trapped gases." Rev. Mod. Phys. 71, 463 (1999).

[5] M. H. Anderson, J. R. Ensher, M. R. Matthews, C. E. Wieman, and E. A. Cornell. “Observation of Bose-Einstein condensation in a dilute atomic vapor.” Science 269, 198 (1995).

[6] K. Davis, M.-O. Mewes, M. Andrews, M. van Druten, D. Durfee, D. Kurn, and W. Ketterle. “Bose-Einstein condensation in a gas of sodium atoms.” Phys. Rev. Lett. 75, 3969 (1995).

[7] C. C. Bradley, C. A. Sackett, J. J. Tollett, and R. G. Hulet. "Evidence of Bose-Einstein condensation in an atomic gas with attracktive interactions.” Phys. Rev. Lett. 75, 1687 (1995).

[8] R. P. Feynman. "Simulating physics with computers." Int. J. Theo. Phys. 21, 467 (1982).

[9] L. V. Hau, S. E. Harris, Z. Dutton, and C. H. Behroozi. "Light speed reduction to 17 metres per second in an ultracold atomic gas.” Nature 397, 594 (1999).

[10] W. Hofstetter, J. I. Cirac, P. Zoller, E. Demler, and M. D. Lukin. "High-temperature superfluidity of fermionic atoms in optical lattices.” Phys. Rev. Lett. 89, 220407 (2002).

[11] M. Greiner, O. Mandel, T. Esslinger, T. W. Hänsch, and I. Bloch. "Quantum phase transition from a superfluid to a Mott insulator in a gas of ultracold atoms." Nature 415, 39 (2002). 
[12] L. Fallani, J. E. Lye, V. Guarrera, C. Fort, and M. Inguscio. "Ultracold atoms in a disordered crystal of light: Towards a Bose glass." Phys. Rev. Lett. 98, 130404 (2007).

[13] H. J. Metcalf and P. van der Straten. Laser Cooling and Trapping. Springer, New York (1999).

[14] R. Shankar. Principles of Quantum Mechanics. Plenum Press, second edition (1994).

[15] J. Weiner. Cold and Ultracold Collisions in Quantum Microscopic and Mesoscopic Systems. Cambridge University Press (2003).

[16] A. J. Leggett. "Bose-Einstein condensation in the alkali gases: Some fundamental concepts.” Rev. Mod. Phys. 73, 307 (2001).

[17] E. Hecht. Optics. Addison Wesley, second edition (2002).

[18] A. A. Michelson and E. W. Morley. "On the relative motion of the earth and the luminiferous ether." Am. Jour. Sci. 34, 333 (1887).

[19] L.-V. de Broglie. Recherches sur la théorie des Quanta. Ph.D. thesis, Paris (1924).

[20] L. H. Germer. “Optical experiments with electrons.” J. Chem. Educ. 5, 1041 (1928).

[21] I. Estermann and O. Stern. “Beugung von Molekularstrahlen.” Z. Phys. 61, 95 (1930).

[22] G. Möllenstedt and H. Düker. "Fresnelscher Interferenzversuch mit einem Biprisma für Elektronenwellen.” Naturwiss. 42, 41 (1954).

[23] C. S. Adams, M. Sigel, and J. Mlynek. “Atom optics.” Phys. Rep. 240, 143 (1994).

[24] P. R. Berman, ed. Atom Interferometry. Academic Press, San Diego (1996).

[25] G. Sagnac. "L'éther lumineux démontré par l'effet du vent relatif d'éther dans un interféromètre en rotation uniforme.” Comptes Rendus 157, 708 (1913).

[26] G. Sagnac. "Sur la preuve de la réalité de l'éther lumineux par l'expérience de l'interférographe tournant." Comptes Rendus 157, 1410 (1913). 
[27] G. E. Stedman. "Ring-laser tests of fundamental physics and geophysics." Rep. Prog. Phys. 60, 615 (1997).

[28] T. L. Gustavson, A. Landragin, and M. A. Kasevich. "Rotation sensing with a dual atominterferometer sagnac gyroscope.” Class. and Quantum Grav. 17, 2385 (2000).

[29] M. R. Andrews, C. G. Townsend, H.-J. Miesner, D. S. Durfee, D. M. Kurn, and W. Ketterle. “Observation of interference between two Bose condensates.” Science 275, 637 (1997).

[30] E. W. Streed, A. P. Chikkatur, T. L. Gustavson, M. Boyd, Y. Torii, D. Schneble, G. K. Campbell, D. E. Pritchard, and W. Ketterle. "Large atom number Bose-Einstein condensate machines.” Rev. of Sci. Instrum. 77, 023106 (2006).

[31] A. Peters, K. Y. Chung, and S. Chu. "High-precision gravity measurements using atom interferometry." Metrologia 38, 25 (2001).

[32] J. M. Reeves. An Atom Waveguide For Interferometry With A Bose-Einstein Condensate of $R b$-87. Ph.D. thesis, University of Virginia (2006).

[33] O. Garcia-Salazar. Bose-Einstein-Condensate Interferometer with Macroscopic Arm Separation. Ph.D. thesis, University of Virginia (2007).

[34] M. D. Barrett, J. A. Sauer, and M. S. Chapman. "All-optical formation of an atomic BoseEinstein condensate." Phys. Rev. Lett. 87, 010404 (2001).

[35] H. J. Lewandowski, D. M. Harber, D. L. Whitaker, and E. A. Cornell. "Simplified system for creating a Bose-Einstein Condensate." J. Low Temp. Phys. 132, 309 (2003).

[36] C. Cohen-Tannoudji, B. Diu, and F. Laloë. Quantum Mechanics. Hermann, Paris, second edition (1977).

[37] W. H. Wing. "On neutral particle trapping in quasistatic electromagnetic fields." Prog. Quant. Elec. 8, 181 (1984). 
[38] E. Majorana. "Atomi orientati in campo magnetico variabile." Nuovo Cimento 9, 43 (1932).

[39] W. Petrich, M. H. Anderson, J. R. Ensher, and E. A. Cornell. "Stable, tightly confining magnetic trap for evaporative cooling of neutral atoms.” Phys. Rev. Lett. 74, 3352 (1995).

[40] W. Ketterle and N. J. van Druten. "Evaporative cooling of atoms." In B. Bederson and H. Walther, eds., Adv. At. Mol. Opt. Phys., volume 37, pp. 181-236. Academic Press, San Diego (1996).

[41] J. Söding, D. Guery-Odelin, P. Desbiolles, F. Chevy, H. Inamori, and J. Dalibard. “Interference of Bose-Einstein condensates split with an atom chip.” Phys. Rev. A 72, 021604(R) (2005).

[42] W. Ketterle, D. S. Durfee, and D. M. Stamper-Kurn. "Making, probing and understanding Bose-Einstein condensates." In Proceedings of the International School of Physics 'Enrico Fermi' Course CXL. IOS Press (1999).

[43] T. L. Gustavson, P. Bouyer, and M. A. Kasevich. "Precision rotation measurements with an atom interferometer gyroscope.” Phys. Rev. Lett. 78, 2046 (1997).

[44] C. R. Ekstrom, J. Schmiedmayer, M. S. Chapman, T. D. Hammond, and D. E. Pritchard. "Measurement of the electric polarizability of sodium with an atom interferometer." Phys. Rev. A 51, 3883 (1995).

[45] S. Gupta, K. W. Murch, K. L. Moore, T. P. Purdy, and D. M. Stamper-Kurn. “Bose-Einstein condensation in a circular waveguide." Phys. Rev. Lett. 95, 143201 (2005).

[46] A. S. Arnold, C. S. Garvie, and E. Riis. "Large magnetic storage ring for Bose-Einstein condensates." Phys. Rev. A 73, 041606 (2006).

[47] C. A. Sackett. "Limits on weak magnetic confinement of neutral atoms." Phys. Rev. A 73, 013626 (2006). 
[48] K. L. Baranowski and C. A. Sackett. "A stable ac current source for magnetic traps." J. Phys. B: At. Mol. Opt. Phys. 39, 2949 (2006).

[49] P. J. Martin, B. G. Oldaker, A. H. Miklich, and D. E. Pritchard. "Bragg scattering of atoms from a standing light wave.” Phys. Rev. Lett. 60, 515 (1988).

[50] D. M. Giltner, R. W. McGowan, and S. A. Lee. "Theoretical and experimental study of the Bragg scattering of atoms from a standing light wave.” Phys. Rev. A 52, 3966 (1995).

[51] P. L. Gould, G. A. Ruff, and D. E. Pritchard. "Diffraction of atoms by light: The near-resonant Kapitza-Dirac effect.” Phys. Rev. Lett. 56, 827 (1986).

[52] Y. Torii, Y. Suzuki, M. Kozuma, T. Sugiura, T. Kuga, L. Deng, and E. W. Hagley. "MachZehnder Bragg interferometer for a Bose-Einstein condensate.” Phys. Rev. A 61, 041602 (2000).

[53] Y.-J. Wang, D. Z. Anderson, V. M. Bright, E. A. Cornell, Q. Diot, T. Kishimoto, M. Prentiss, R. A. Saravanan, S. R. Segal, and S. Wu. "An atom Michelson interferometer on a chip using a Bose-Einstein Condensate.” Phys. Rev. Lett. 94, 090405 (2005).

[54] S. Wu, E. J. Su, and M. Prentiss. "Time domain de Broglie wave interferometry along a magnetic guide.” Eur. Phys. J. D 35, 111 (2005).

[55] O. Garcia, B. Deissler, K. J. Hughes, J. M. Reeves, and C. A. Sackett. "Bose-Einstein condensate interferometer with macroscopic arm separation.” Phys. Rev. A 74, 031601(R) (2006).

[56] M. Horikoshi and K. Nakagawa. "Dephasing due to atom-atom interaction in a waveguide interferometer using a Bose-Einstein condensate." Physical Review A (Atomic, Molecular, and Optical Physics) 74, 031602 (2006).

[57] S. Inouye, T. Pfau, S. Gupta, A. P. Chikkatur, A. Görlitz, D. E. Pritchard, and W. Ketterle. "Phase-coherent amplification of atomic matter waves." Nature 402, 641 (1999). 
[58] E. W. Hagley, L. Deng, M. Kozuma, J. Wen, K. Helmerson, S. L. Rolston, and W. D. Phillips. “A well-collimated quasi-continuous atom laser.” Science 283, 1706 (1999).

[59] T. Kinoshita, T. Wenger, and D. S. Weiss. “A quantum Newton's cradle.” Nature 440, 900 (2006).

[60] S. Wu, Y.-J. Wang, Q. Diot, and M. Prentiss. "Splitting matter waves using an optimized standing-wave light-pulse sequence.” Phys. Rev. A 71, 043602 (2005).

[61] P. Meystre and M. S. III. Elements of Quantum Optics. Springer, Berlin, third edition (1999).

[62] R. P. Feynman, J. Frank L. Vernon, and R. W. Hellwarth. "Geometrical representation of the Schrödinger equation for solving maser problems.” J. Appl. Phys. 28, 49 (1957).

[63] K. J. Hughes, B. Deissler, J. H. T. Burke, and C. A. Sackett. "High-fidelity manipulation of a Bose-Einstein condensate using an optical standing wave.” Phys. Rev. A 76, 035601 (2007).

[64] Y. Shin, M. Saba, T. A. Pasquini, W. Ketterle, D. E. Pritchard, and A. E. Leanhardt. "Atom interferometry with Bose-Einstein condensates in a double-well potential.” Phys. Rev. Lett. 92, 050405 (2004).

[65] G.-B. Jo, Y. Shin, S. Will, T. A. Pasquini, M. Saba, W. Ketterle, D. E. Pritchard, M. Vengalattore, and M. Prentiss. "Long phase coherence time and number squeezing of two Bose-Einstein condensates on an atom chip.” Phys. Rev. Lett. 98, 030407 (2007).

[66] J. M. McGuirk, G. T. Foster, J. B. Fixler, M. J. Snadden, and M. A. Kasevich. "Sensitive absolute-gravity gradiometry using atom interferometry." Phys. Rev. A 65, 033608 (2002).

[67] A. Wicht, J. M. Hensley, E. Sarajlic, and S. Chu. "A preliminary measurement of the fine structure constant based on atom interferometry.” Phys. Scr. T102, 82 (2002).

[68] A. Miffre, M. Jacquey, M. Büchner, G. Trénec, and J. Vigué. "Measurement of the electric polarizability of lithium by atom interferometry." Phys. Rev. A 73, 011603 (2006). 
[69] J. Schmiedmayer, M. S. Chapman, C. R. Ekstrom, T. D. Hammond, S. Wehinger, and D. E. Pritchard. "Index of refraction of various gases for sodium matter waves." Phys. Rev. Lett. 74, 1043 (1995).

[70] J. D. Perreault and A. D. Cronin. "Observation of atom wave phase shifts induced by van der Waals atom-surface interactions.” Phys. Rev. Lett. 95, 133201 (2005).

[71] S. Gupta, K. Dieckmann, Z. Hadzibabic, and D. E. Pritchard. "Contrast interferometry using Bose-Einstein condensates to measure h/m and $\alpha$.” Phys. Rev. Lett. 89, 140401 (2002).

[72] M. Saba, T. A. Pasquini, C. Sanner, Y. Shin, W. Ketterle, and D. E. Pritchard. "Light scattering to determine the relative phase of two Bose-Einstein condensates.” Science 307, 1945 (2005).

[73] G. Nogues, A. Rauschenbeutel, S. Osnaghi, M. Brune, J. M. Raimond, and S. Haroche. "Seeing a single photon without destroying it." Nature 400, 239 (1999).

[74] F. Shimizu. "Specular reflection of very slow metastable neon atoms from a solid surface." Phys. Rev. Lett. 86, 987 (2001).

[75] T. A. Pasquini, Y. Shin, C. Sanner, M. Saba, A. Schirotzek, D. E. Pritchard, and W. Ketterle. “Quantum reflection from a solid surface at normal incidence.” Phys. Rev. Lett. 93, 223201 (2004).

[76] L. Mandel and E. Wolf. Optical coherence and quantum optics. Cambridge University Press (1995).

[77] J. Javanainen and M. Wilkens. "Phase and phase diffusion of a split Bose-Einstein condensate." Phys. Rev. Lett. 78, 4675 (1997).

[78] M. Olshanii. "Atomic scattering in the presence of an external confinement and a gas of impenetrable bosons.” Phys. Rev. Lett. 81, 938 (1998). 
[79] M. Olshanii and V. Dunjko. "Interferometry in dense nonlinear media and interactioninduced loss of contrast in microfabricated atom interferometers.” (2005). ArXiv:condmat/0505358.

[80] J. A. Stickney, D. Z. Anderson, and A. A. Zozulya. "Increasing the coherence time of BoseEinstein-condensate interferometers with optical control of dynamics.” Phys. Rev. A 75, 063603 (2007).

[81] E. L. Hahn. “Spin echoes.” Phys. Rev. 80, 580 (1950).

[82] J. A. Stickney, R. P. Kafle, D. Z. Anderson, and A. A. Zozulya. “Theoretical analysis of a single and double reflection atom interferometer in a weakly-confining magnetic trap.” (2007). ArXiv:0712.2057.

[83] S. R. Segal, Q. Diot, E. A. Cornell, M. Prentiss, A. A. Zozulya, and D. Z. Anderson. “On-chip Bose-Einstein condensate interferometer with $0.5 \mathrm{~mm}$ arm length.” In Bull. Am. Phys. Soc., volume 52. American Physical Society (2007).

[84] M. Horikoshi and K. Nakagawa. "Suppression of dephasing due to a trapping potential and atom-atom interactions in a trapped-condensate interferometer.” Phys. Rev. Lett.99, 180401 (2007).

[85] K. Bongs and K. Sengstock. "Physics with coherent matter waves.” Rep. Prog. Phys. 67, 907 (2004).

[86] H. Kreutzmann, U. V. Poulsen, M. Lewenstein, R. Dumke, W. Ertmer, G. Birkl, and A. Sanpera. “Coherence properties of guided-atom interferometers.” Phys. Rev. Lett. 92, 163201 (2004).

[87] T. Schumm, S. Hofferberth, L. M. Andersson, S. Wildermuth, S. Groth, I. Bar-Joseph, J. Schmiedmayer, and P. Krüger. "Matter-wave interferometry in a double well on an atom chip." Nature Phys. 1, 57 (2005). 
[88] U. Sterr, K. Sengstock, J. H. Müller, D. Bettermann, and W. Ertmer. “The magnesium Ramsey interferometer: Applications and prospects.” Appl. Phys. B 54, 341 (1992).

[89] A. Morinaga, T. Tako, and N. Ito. "Sensitive measurement of phase shifts due to the ac Stark effect in a Ca optical Ramsey interferometer." Phys. Rev. A 48, 1364 (1993).

[90] M. A. Kadar-Kallen and K. D. Bonin. "Light-force technique for measuring polarizabilities.” Phys. Rev. Lett. 68, 2015 (1992).

[91] J. Javanainen. "Optical signatures of a tightly confined Bose condensate." Phys. Rev. Lett. 72, 2375 (1994).

[92] L. You, M. Lewenstein, R. J. Glauber, and J. Cooper. "Quantum field theory of atoms interacting with photons. III. Scattering of weak cw light from cold samples of bosonic atoms." Phys. Rev. A 53, 329 (1996).

[93] C. E. Wieman, M. C. Noecker, B. P. Masterson, and J. Cooper. "Asymmetric line shapes for weak transitions in strong standing-wave fields.” Phys. Rev. Lett. 58, 1738 (1987).

[94] P. R. Hemmer, M. S. Shahriar, V. D. Natoli, and S. Ezekiel. "Ac Stark shifts in a two-zone Raman interaction.” J. Opt. Soc. Am. B 6, 1519 (1989).

[95] M. S. Safronova and C. W. Clark. "Inconsistencies between lifetime and polarizability measurements in Cs.” Phys. Rev. A 69, 040501 (2004).

[96] R. Grimm, M. Weidemüller, and Y. B. Ovchinnikov. "Optical dipole traps for neutral atoms.” In B. Bederson and H. Walther, eds., Adv. At., Mol., Opt. Phys., volume 42, pp. 95-170. Academic Press, San Diego (2000).

[97] P. Staanum and M. Drewsen. “Trapped-ion quantum logic utilizing position-dependent ac Stark shifts.” Phys. Rev. A 66, 040302 (2002).

[98] J. D. Jackson. Classical Electrodynamics. John Wiley and Sons, Inc., third edition (1999). 
[99] C. Chicone. Ordinary Differential Equations with Applications. Springer, New York (1999).

[100] S.-I. Chu. "Recent developments in semiclassical Floquet theories for intense-field multiphoton processes." In S. D. Bates and B. Bederson, eds., Adv. At. Mol. Phys., volume 21, pp. 197-253. Academic Press, Orlando (1985).

[101] R. M. Potvliege and R. Shakeshaft. "Nonperturbative calculation of partial differential rates for multiphoton ionization of a hydrogen atom in a strong laser field." Phys. Rev. A 38, 1098 (1988).

[102] Y. Ralchenko, F.-C. Jou, D. Kelleher, A. Kramida, A. Musgrove, J. Reader, W. Wiese, and K. Olsen. “NIST Atomic Spectra Database (version 3.1.3), [online].” (2007). URL http: //physics.nist.gov/asd3

[103] U. Volz and H. Schmoranzer. "Precision lifetime measurements on alkali atoms and on helium by beam-gas-laser spectroscopy.” Physica Scripta T65, 48 (1996).

[104] R. Kurucz and B. Bell. 1995 Atomic Line Data. Kurucz CD-ROM No. 23. Cambridge, Mass.: Smithsonian Astrophysical Observatory.

[105] I. Vayshenker, H. Haars, X. Li, J. H. Lehman, and D. J. Livigni. “Comparison of opticalpower meters between the NIST and the PTB.” Metrologia 37, 349 (2000).

[106] A. Kastler. “Displacement of energy levels of atoms by light.” J. Opt. Soc. Am. 53, 902 (1963).

[107] C. Cohen-Tannoudji, J. Dupont-Roc, and G. Grynberg. Atom-Photon Interactions: Basic Processes and Applications. Wiley, New York (1992).

[108] R. H. Dicke. “Coherence in spontaneous radiation processes.” Phys. Rev. 93, 99 (1954).

[109] M. Gross and S. Haroche. "Superradiance: An essay on the theory of collective spontaneous emission." Phys. Rep. 93, 301 (1982). 
[110] S. Inouye, A. P. Chikkatur, D. M. Stamper-Kurn, J. Stenger, D. E. Pritchard, and W. Ketterle. "Superradiant Rayleigh scattering from a Bose-Einstein condensate." Science 285, 571 (1999).

[111] A. Lagendijk and B. A. van Tiggelen. "Resonant multiple scattering of light.” Phys. Rep. 270, 143 (1996).

[112] R. Loudon. The Quantum Theory of Light. Oxford University Press, third edition (2000).

[113] T. M. Miller and B. Bederson. "Atomic and molecular polarizabilities - a review of recent advances.” In D. R. Bates and B. Bederson, eds., Adv. At. Mol. Phys., volume 13, pp. 1-55. Academic Press, New York (1977).

[114] R. W. Molof, H. L. Schwartz, T. M. Miller, and B. Bederson. "Measurements of electric dipole polarizabilities of the alkali-metal atoms and the metastable noble-gas atoms." Phys. Rev. A 10, 1131 (1974).

[115] J. M. Amini and H. Gould. "High precision measurement of the static dipole polarizability of cesium.” Phys. Rev. Lett. 91, 153001 (2003).

[116] C. Fertig and K. Gibble. "Measurement and cancellation of the cold collision frequency shift in an ${ }^{87}$ Rb fountain clock." Phys. Rev. Lett. 85, 1622 (2000).

[117] L. Maleki and J. Prestage. "Applications of clocks and frequency standards: from the routine to tests of fundamental models.” Metrologia 42, S145 (2005).

[118] J. Camparo. “The rubidium atomic clock and basic research.” Physics Today 60, 33 (2007).

[119] W. M. Itano, L. L. Lewis, and D. J. Wineland. "Shift of ${ }^{2} s_{1 / 2}$ hyperfine splittings due to blackbody radiation.” Phys. Rev. A 25, 1233 (1992).

[120] E. J. Angstmann, V. A. Dzuba, and V. V. Flambaum. "Frequency shift of the cesium clock transition due to blackbody radiation." Phys. Rev. Lett. 97, 040802 (2006). 
[121] A. D. Cronin, J. Schmiedmayer, and D. E. Pritchard. "Atom interferometers." (2007). ArXiv:0712.3703.

[122] S. Wu, E. Su, and M. Prentiss. "Demonstration of an area-enclosing guided-atom interferometer for rotation sensing." Phys. Rev. Lett. 99, 173201 (2007).

[123] B. Deissler. A Magnetic Trap for Evaporative Cooling of Rb Atoms. Master's thesis, University of Virginia (2003). 